\title{
Generalized Volatility-Stabilized Processes
}

\author{
Radka Picková
}

Submitted in partial fulfillment of the

\author{
Requirements for the degree \\ of Doctor of Philosophy
}

in the Graduate School of Arts and Sciences

\section{COLUMBIA UNIVERSITY}


(C) 2013

Radka Picková

All Rights Reserved 


\title{
ABSTRACT
}

\section{Generalized Volatility-Stabilized Processes}

\author{
Radka Picková
}

In this thesis, we consider systems of interacting diffusion processes which we call Generalized Volatility-Stabilized processes, as they extend the Volatility-Stabilized Market models introduced in Fernholz and Karatzas (2005). First, we show how to construct a weak solution of the underlying system of stochastic differential equations. In particular, we express the solution in terms of time-changed squared-Bessel processes and argue that this solution is unique in distribution. In addition, we also discuss sufficient conditions under which this solution does not explode in finite time, and provide sufficient conditions for pathwise uniqueness and for existence of a strong solution.

Secondly, we discuss the significance of these processes in the context of Stochastic Portfolio Theory. We describe specific market models which assume that the dynamics of the stocks' capitalizations is the same as that of the Generalized Volatility-Stabilized processes, and we argue that strong relative arbitrage opportunities may exist in these markets, specifically, we provide multiple examples of portfolios that outperform the market portfolio. Moreover, we examine the properties of market weights as well as the diversity weighted portfolio in these models. 
Thirdly, we provide some asymptotic results for these processes which allows us to describe different properties of the corresponding market models based on these processes. 


\section{Contents}

Chapter 2: System of SDEs for the Generalized Volatility-Stabilized Processes 4

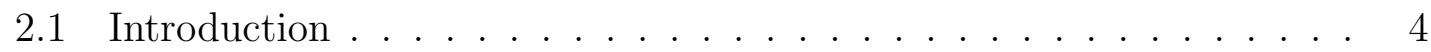

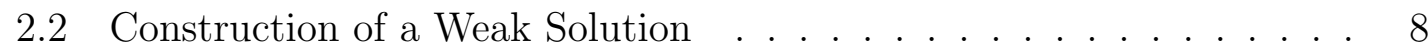

2.2.1 Solution of the SDEs - A Simple Case First:

$\alpha_{1}=\ldots=\alpha_{n}=0, \sigma=1$ and $K(\cdot) \equiv 1 \ldots \ldots \ldots$

2.2.2 Solution of the SDEs - The General Case . . . . . . . . . . . 21

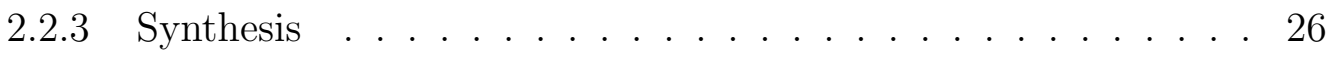

2.3 Discussion of conditions on $K(\cdot) \quad \ldots \ldots \ldots \ldots$

2.3.1 Growth Conditions on $K(\cdot)$ to ensure property $(\mathrm{P} 1) \ldots \ldots$

2.3.2 Conditions on $K(\cdot)$ to ensure property $(\mathrm{P} 2) \ldots \ldots . \ldots 34$

2.4 Pathwise Uniqueness and Strength _ . . . . . . . . . . . 35

Chapter 3: Generalized Volatility-Stabilized processes in Stochastic $\begin{array}{ll}\text { Portfolio Theory } & 40\end{array}$

3.1 Basic Concepts of Stochastic Portfolio Theory . . . . . . . . . . . . 41 
3.2 Arbitrage Opportunities in Generalized

Volatility-Stabilized Markets . . . . . . . . . . . . . . . . 45

3.3 Market Weights and the Diversity Weighted Portfolio . . . . . . . . . 55

Chapter 4: Asymptotics for the Generalized Volatility-Stabilized Processes

4.1 Some Asymptotic Results - the Case of $\alpha=0$, that is $m=2 \ldots \ldots \ldots \ldots$. . . . . . . . . . . . . .

4.2 Some Asymptotic Results - the Case of $\alpha>0$, that is $m>2 \ldots \ldots 66$

4.3 Proofs of $(4.4)$ and $(4.6) \ldots \ldots \ldots \ldots$. . . . . . . . . . . 67

4.3.1 Proof of $(4.4) \ldots \ldots \ldots$. . . . . . . . . . . . 67

4.3.2 Proof of $(4.6) \ldots \ldots \ldots \ldots$. . . . . . . . . . . . 70

$\begin{array}{ll}\text { Chapter 5: Conclusion and future directions } & 76\end{array}$

5.1 Study of explosions . . . . . . . . . . . . . . . 76

5.2 Invariant distribution of the market weights . . . . . . . . . 80

5.3 Other possible extensions . . . . . . . . . . . . . . . 81

$\begin{array}{lr}\text { Bibliography } & 81\end{array}$

Appendix A: Bessel and Squared-Bessel Processes $\quad 84$

A.1 Definition and Basic Properties . . . . . . . . . . . . . . 84 


\section{Acknowledgments}

This dissertation concludes five wonderful years of my doctoral studies at the Department of Statistics at Columbia University. I would like to thank everyone who has walked with me on this journey and helped made it so pleasant and enriching experience.

My deepest gratitude is to my advisor, Professor Ioannis Karatzas, who has been the most motivating and inspiring person during my whole studies. I very much enjoyed being a student at his lectures on Stochastic Analysis and its Applications, and have been admiring his enthusiasm and dedication for research and teaching ever since then. This work would have not been possible without his immense support and thoughtful guidance. I thank him for having been patient with me, for always providing immediate feedback and suggestions, and having been available whenever I needed despite his busy schedule.

I am very grateful that I have been invited to attend meetings of the research group at Intech in Princeton where numerous fruitful discussions have substantially improved my understanding of the field of Stochastic Portfolio Theory and various related problems. I thank Dr. Robert Fernholz, Dr. Adrian Banner, Dr. Jonathan Hanke, Professor Tomoyuki Ichiba, Professor Soumik Pal, Dr. Vasileios Papathanakos, Dr. Johannes Ruf, Dr. Subhankar Sadhukhan, Dr. Mykhaylo Shkol- 
nikov, Dr. Winslow Strong, and Dr. Phillip Whitman, for sharing their insights and ideas with me, and for providing feedback on my work.

I am very thankful to Professor Jose H. Blanchet, Professor Victor H. de la Pena, Professor Julien Dubedat, and Professor Philip E. Protter for serving on my defense committee. I am especially thankful to Professor Philip E. Protter for having been very supportive and his helpful suggestions.

I am grateful to all Professors at the Department of Statistics at Columbia University who have been my teachers, mentors and instructors, and who have taught me how to be a better scholar and researcher. I thank the department's chair, Professor David Madigan, and the director of the graduate studies, Professor Richard A. Davis, for creating very friendly and creative atmosphere at the department. I thank my teachers: Professor Victor de la Pena, Professor Andrew Gelman, Professor Jingchen Liu, Professor Duong Hong Phong, Professor Zhiliang Ying, and Professor Jan Večer. I also thank my teachers from other departments at Columbia: most importantly Professor Rama Cont, and Professor Tim Siu-Tang Leung. I am grateful to Professor Mark Brown, Professor Olympia Hadjiliadis, and Professor Michael Hogan, who all have been very supportive and provided valuable feedback on my work.

I am also thankful to the staff at the Department of Statisics, Anthony and Dood, for their help, support and friendliness during my studies at Columbia.

I am grateful to Columbia University and the National Science Foundation (DMS Grant 09-05754) for their financial support.

I am grateful to all my classmates, friends and colleagues at the statistics department, who have made my years at Columbia University full of fond mem- 
ories. I especially thank my senior classmates Amal, Francisco, Georgios, Gerardo, Johannes, Libor, Mladen, Petr, Shawn, Subhankar, and Tyler, whose friendship I deeply appreciate. My cohort: Chien-Hsun, Gongjun, Heng, Junyi, Katya, Meghan, Nick, Pengfei, Shane, Tony, Vince, Yongbum, with whom I spent numerous hours solving homework problems and preparing for the qualifying exams. Warm thoughts to Ben, Diego, Jingjing, Kristen, Louis, Maria, Rohit, Roseline, Samantha, Stephanie, Wei, Yi, and Zach.

I am also thankful to my friends from other departments at Columbia University: Ale, Andrei, Arsenyi, Aya, Berk, Chris, Emrah, and Nikiforos, with whom I shared ups and downs of our lives as PhD students in New York City.

Warm thoughts go to my friends and teammates from Columbia cycling team: Aimee, Corey, Francesco, Isaac, James, Jim, Joe, Liz, Nicole, Sam, Shane, and Rob; the time spent with them while riding bikes and racing ECCC in Columbia blue will always stay in my memory.

I am very grateful to my Czech friends that I met at Columbia: Honza, Libor, Martina, and Petr, who became my close friends while they were helping me to adjust to and enjoy living in New York City.

I am also thankful to my colleagues at Morgan Stanley, especially Kashi Behrstock, Stefan Andreev and other members of the interest rates strats group, with whom I worked during my two internships and who showed me how to apply my knowledge in real-life financial applications.

Studying in a PhD program would have been much harder without the proper background I gained during the bachelor and master study programs at the Charles University in Prague. I would like to take this opportunity to thank again my 
diploma thesis advisor, Professor Jitka Dupačová, my bachelor thesis advisor, Professor Zdeněk Hlávka, as well as many other Professors at the Faculty of Mathematics and Physics who inspired me to pursue an academic career and whose lectures I still keep in my memories.

I would also like to thank my teachers at Gymnázium Dr. J. Pekaře, especially Petr Kasal and Jarmila Mulačová, as they encouraged me in my interest in mathematics and physics.

I am grateful to my friends in the Czech Republic who supported me and stayed close to me despite the ocean in between us: Tomáš, Pétăa, Barča, Boban, Zbyňa, Marek, Unkl Dzou, and Velká Pusa.

Finally, and most importantly, I am extremely grateful to my parents, Karel and Miloslava, and my brother Karel for their unconditional love and support. None of my achievements would have happened without them. My warmest thought is to my aunt Hana, and my cousins Johana and Kateřina. 
To Miloslava and Karel

In memory of my uncle Josef 


\section{Chapter 1}

\section{Outline of Thesis}

In this thesis, we introduce and study in detail specific systems of interacting diffusion processes which we call Generalized Volatility-Stabilized processes. These processes extend the Volatility-Stabilized Market models which were introduced in Fernholz and Karatzas (2005) in a nontrivial way so that a wide variety of models is covered in this class of models.

In the first part of the thesis, that is in Chapter 2, we prove that there exists a weak solution of the underlying systems of stochastic differential equations, even though one cannot use any of the standard theorems for proving existence of a solution to a system of stochastic differential equations. To be more specific, we show that it is possible to construct a weak solution using a method of time-change and scaling, and express it in terms of time-changed squared-Bessel processes. In addition, we argue that the constructed solution is unique in distribution, and we state sufficient conditions under which the solution does not explode in finite time. We also provide sufficient conditions under which pathwise uniqueness holds for the system, and hence allows us to argue the existence of a strong solution.

The second part of the thesis, that is Chapter 3, is devoted to the discussion

of the significance of the Generalized Volatility-Stabilized processes in the context of 
Stochastic Portfolio Theory which was founded by Dr. Robert Fernholz. Multiple papers that he, and his collaborators, wrote in the eighties and nineties (we do not attempt to write a list of the references here, as such a list would surely be incomplete) lead him to write the monograph Fernholz (2002) which provides a great introduction into this new approach to understanding the markets behavior and portfolio management, and also includes many interesting examples and a number of open problems. Another good introduction to this framework is the overview paper Fernholz and Karatzas (2009), which provided an update regarding the solved problems and also lists references to literature that had been written with related topics.

In short, Stochastic Portfolio Theory attempts to understand the structure and behavior of real equity markets without making any normative assumptions, as is common in classical mathematical finance. For instance, Stochastic Portfolio Theory does not make a priori the assumption that there is no arbitrage in the markets, on the contrary, it studies which conditions lead to existence of arbitrage opportunities in market models that are consistent with characteristics observed in actual markets. We discuss in detail the properties of specific market models which assume that the dynamics of the stocks' capitalizations is the same as that of the Generalized Volatility-Stabilized processes, and we argue that strong relative arbitrage opportunities may exist in these markets, specifically, we provide multiple examples of portfolios that outperform the market portfolio. Moreover, we examine the properties of market weights as well as the diversity weighted portfolio in these models.

In the third part of the thesis, that is Chapter 4, we provide some asymptotic results for these processes which allows us to describe different properties of the corresponding market models based on these processes.

Chapter 5 contains a summary of some of the related open questions, exten- 
sions and possible future work.

Since many results in this thesis make use of the properties of Bessel and squared-Bessel processes we include, at the end of the thesis, in the Appendix, a section with definitions, basic properties and some known results for Bessel and squared-Bessel processes in order to make it easily accessible to the reader.

Some of the results presented in this thesis are going to appear in the Annals of Finance, in the paper Pickova (2013). 
Chapter 2. System of SDEs for the Generalized Volatility-Stabilized

\section{Chapter 2}

\section{System of SDEs for the \\ Generalized Volatility-Stabilized \\ Processes}

\section{$2.1 \quad$ Introduction}

Let us consider a vector process $X(t)=\left(X_{1}(t), \ldots, X_{n}(t)\right), t \in[0, \infty)$ with values in the state space $(0, \infty)^{n}$, that solves the following system of stochastic differential equations

$$
\begin{aligned}
\mathrm{d}\left(\log X_{i}(t)\right) & =\frac{\alpha_{i}}{2\left(\mu_{i}(t)\right)^{2 \beta}}[K(X(t))]^{2} \mathrm{~d} t+\frac{\sigma}{\left(\mu_{i}(t)\right)^{\beta}} K(X(t)) \mathrm{d} W_{i}(t), \\
X_{i}(0) & =x_{i}>0, \quad i=1, \ldots, n .
\end{aligned}
$$

Here $\alpha_{i} \geq 0, \sigma>0, \beta>0$ are given real numbers, $\mu(\cdot)=\left(\mu_{1}(\cdot), \ldots, \mu_{n}(\cdot)\right)$ is the vector of so-called market weights (see Section 3.1 for explanation of this terminology)

$$
\mu_{i}(t)=\frac{X_{i}(t)}{X_{1}(t)+\cdots+X_{n}(t)}, \quad i=1, \ldots, n,
$$


the given function $K(\cdot):(0, \infty)^{n} \rightarrow(0, \infty)$ is measurable, and $W_{1}(\cdot), \ldots, W_{n}(\cdot)$ are independent Brownian motions. Sufficient conditions on $K(\cdot)$ so that the system in (2.1) has a weak solution that is unique in distribution and does not explode in finite time will be provided in Section 2.3 below. Moreover, sufficient conditions for pathwise uniqueness and for existence of a strong solution for the system of equations (2.1) are stated in Section 2.4. Introducing the function $\mathcal{T}(\cdot):(0, \infty)^{n} \rightarrow(0, \infty)$ given by

$$
\mathcal{T}(x):=\left(\sum_{i=1}^{n} x_{i}\right)^{\beta} K(x), \quad x \in(0, \infty)^{n},
$$

we shall seek a solution to the equivalent system of stochastic differential equations

$$
\mathrm{d} X_{i}(t)=\frac{\alpha_{i}+\sigma^{2}}{2}\left[X_{i}(t)\right]^{1-2 \beta}[\mathcal{T}(X(t))]^{2} \mathrm{~d} t+\sigma\left[X_{i}(t)\right]^{1-\beta} \mathcal{T}(X(t)) \mathrm{d} W_{i}(t)
$$

for $i=1, \ldots, n$, with state space $(0, \infty)^{n}$ and with $n$ independent Brownian motions $W_{1}(\cdot), \ldots, W_{n}(\cdot)$.

Notice two special cases: first, if $K(\cdot) \equiv 1$ and if we allow $\beta=0$, then the system of equations (2.1) corresponds to the setting, where

$$
X_{i}(t)=x_{i} \mathrm{e}^{(\alpha / 2) t+\sigma W_{i}(t)}, \quad i=1, \ldots, n
$$

are independent Geometric Brownian motions; secondly, the case of $K(\cdot) \equiv 1$ and $\beta=1 / 2$ corresponds to the volatility-stabilized market models, which were introduced and studied by Fernholz and Karatzas (2005), and were studied in further detail by Goia (2009) and Pal (2011).

If $K(\cdot) \equiv 1$ (or any other positive real constant) and $\beta>0$ is arbitrary, it is possible to use the theory of degenerate differential equations developed by Bass and Perkins (2002) and show that the system of equations (2.1) in this case has a weak solution, unique in the sense of the probability distribution. 
Remark: Bass and Perkins (2002) developed a general theory for degenerate differential equations of the same type as the one in (2.1), which for the choice of $K(\cdot) \equiv 1$ has an equivalent form

$$
\begin{aligned}
& \mathrm{d} X_{i}(t)= \frac{\alpha_{i}+\sigma^{2}}{2}\left(X_{i}(t)\right)^{1-2 \beta}\left(X_{1}(t)+\ldots+X_{n}(t)\right)^{2 \beta} \mathrm{d} t \\
&\left.\quad+\sigma\left(X_{i}(t)\right)^{1-\beta}\left(X_{1}(t)+\ldots+X_{n}(t)\right)^{\beta} \mathrm{d} W_{i}(t), \quad i=1, \ldots, n .5\right)
\end{aligned}
$$

With the notation $D_{i}=\frac{\partial}{\partial x_{i}}, D_{i j}^{2}=\frac{\partial^{2}}{\partial x_{i} \partial x_{j}}$, they consider the operator

$$
\sum_{i, j=1}^{n} \sqrt{x_{i} x_{j}} \gamma_{i j}(x) D_{i j}^{2}+\sum_{i=1}^{n} b_{i}(x) D_{i}
$$

acting on functions in $\mathcal{C}_{b}^{2}\left(\mathbb{R}_{+}^{n}\right)$, the space of bounded $\mathcal{C}^{2}$ functions on the nonnegative orthant with bounded first- and second- order partial derivatives, and prove wellposedness of the martingale problem for this degenerate operator under suitable nonnegativity and regularity conditions on $\gamma_{i j}(\cdot)$ and $b_{i}(\cdot)$. In particular, $\gamma_{i j}(\cdot)$ and $b_{i}(\cdot)$ need to be Hölder continuous. The infinitesimal generator associated to the system in $(2.5)$ is

$$
\mathcal{A} f(x)=\frac{1}{2}\left(x_{1}+\ldots+x_{n}\right)^{2 \beta} \sum_{i=1}^{n}\left(\sigma^{2} x_{i}^{2-2 \beta} D_{i i}^{2} f(x)+\left(\alpha_{i}+\sigma^{2}\right) x_{i}^{1-2 \beta} D_{i} f(x)\right)
$$

acting on functions in $\mathcal{C}_{b}^{2}\left(\mathbb{R}_{+}^{n}\right)$. Hence, the corresponding $\gamma_{i j}(\cdot)$ and $b_{i}(\cdot)$ are

$$
\begin{gathered}
b_{i}(x)=\frac{1}{2}\left(\alpha_{i}+\sigma^{2}\right)\left(x_{1}+\ldots+x_{n}\right)^{2 \beta} x_{i}^{1-2 \beta}, \\
\gamma_{i i}(x)=\frac{1}{2}\left(\sigma^{2}\right)\left(x_{1}+\ldots+x_{n}\right)^{2 \beta} x_{i}^{1-2 \beta},
\end{gathered}
$$

and $\gamma_{i j}(x)=0, i \neq j$. In the case when $\beta=1 / 2, \gamma_{i i}(\cdot)$ and $b_{i}(\cdot)$ are Hölder continuous, and for other values of $\beta>0$ they are Hölder continuous locally, away from the origin.

With more general (possibly discontinuous) drift and volatility coefficients, the system in (2.1) fails to satisfy the conditions required in Bass and Perkins (2002). 
However, as we will discuss in the following sections (especially in Section 2.2 and 2.3), it is still possible, just as in Fernholz and Karatzas (2005), to construct a weak solution from first principles, and express it in terms of time-changed squared-Bessel processes. We shall describe this construction below and will also argue that under certain assumptions (see Section 2.3) this weak solution is unique in the sense of the probability distribution and does not explode in finite time.

Sufficient assumptions on $K(\cdot)$ so that the system in $(2.1)$ has a weak solution that is unique in distribution and does not explode in finite time are provided in Section 2.3, and are relatively weak, in the sense that the coefficients of the system in (2.1) for these choices of $K(\cdot)$ would not satisfy the classical sufficient conditions for existence and uniqueness of general systems of stochastic differential equations stated in most well-known theorems. Namely, the function $K(\cdot)$ is not even assumed to be continuous, which means that the coefficients of the systems in (2.1) need not be continuous, nor do they need to be bounded, and therefore results of many classical theorems would not apply. For instance, the result of Itô requires Lipschitz continuity of coefficients (see Theorem 2.9 in Karatzas and Shreve (1991)), Skorokhod's theorem also requires continuous, as well as bounded, coefficients (see Theorem 23.5 in Rogers and Williams (2000)), so does Stroock and Varadhan (see Theorem 4.22 in Karatzas and Shreve (1991)), whereas Krylov (1969) does not require continuity but does assume bounded coefficients.

The remainder of this chapter is organized as follows. In Section 2.2, a weak solution to the system in (2.1) is constructed following the steps first of Analysis and consequently of Synthesis. Under certain conditions on the function $K(\cdot)$, this approach also allows to argue that the constructed solution is unique in distribution (respectively, does not explode in finite time). Section 2.3 discusses these conditions on the function $K(\cdot)$ that are sufficient for the existence of a weak solution that is unique in distribution (respectively, for the existence of a non-exploding solution), 
Chapter 2. System of SDEs for the Generalized Volatility-Stabilized

whereas Section 2.4 focuses on conditions on the function $K(\cdot)$ that lead to pathwise uniqueness, and hence to the existence of a strong solution, for the system in (2.1).

\subsection{Construction of a Weak Solution}

In this section, we shall show that it is possible to construct a weak solution of the system in (2.1) using appropriately scaled and time-changed squared-Bessel processes. We will discuss first a simple case, with $\alpha_{1}=\ldots=\alpha_{n}=0, \sigma=1$ and $K(\cdot) \equiv 1$, and then the general case. We shall also argue that under certain conditions the solution is unique in distribution and does not explode in finite time. Indeed, whether these conditions are satisfied in the general case depends on the particular choice of the function $K(\cdot)$, which will be discussed in detail in Section 2.3 .

\subsubsection{Solution of the SDEs - A Simple Case First:$$
\alpha_{1}=\ldots=\alpha_{n}=0, \sigma=1 \text { and } K(\cdot) \equiv 1
$$

Let us try to understand first the system of stochastic differential equations in (3.16) in a simple case with $\alpha_{1}=\ldots=\alpha_{n}=0, \sigma=1$ and $K(\cdot) \equiv 1$, that is,

$$
\begin{aligned}
\mathrm{d}\left(\log X_{i}(t)\right) & =\left(\frac{X_{1}(t)+\ldots+X_{n}(t)}{X_{i}(t)}\right)^{\beta} \mathrm{d} W_{i}(t), \quad i=1, \ldots, n \\
X_{i}(0) & =x_{i}>0, \quad i=1, \ldots, n
\end{aligned}
$$

in the positive orthant $(0, \infty)^{n}$.

Equivalently, we seek a weak solution for the system of stochastic differential equations

$$
\begin{aligned}
\mathrm{d} X_{i}(t)= & \frac{1}{2}\left(X_{i}(t)\right)^{1-2 \beta}\left(X_{1}(t)+\ldots+X_{n}(t)\right)^{2 \beta} \mathrm{d} t \\
& +\left(X_{i}(t)\right)^{1-\beta}\left(X_{1}(t)+\ldots+X_{n}(t)\right)^{\beta} \mathrm{d} W_{i}(t),
\end{aligned}
$$


for $i=1, \ldots, n$, and where the state process $X(\cdot)=\left(X_{1}(\cdot), \ldots, X_{n}(\cdot)\right)$ takes values in $(0, \infty)^{n}$, and $W(\cdot)=\left(W_{1}(\cdot), \ldots, W_{n}(\cdot)\right)$ is an $n$-dimensional Brownian motion.

\subsubsection{Analysis}

Suppose that this system admits a weak solution. Namely, that we can find a filtered probability space $(\Omega, \mathcal{F}, \mathbb{P}), \mathbf{F}=\{\mathcal{F}(t)\}_{0 \leq t<\infty}$, and on it independent Brownian motions $\left(W_{1}(\cdot), \ldots, W_{n}(\cdot)\right)$, as well as continuous $\mathbf{F}$-adapted processes $\left(X_{1}(\cdot), \ldots, X_{n}(\cdot)\right)$ with values in the positive orthant $(0, \infty)^{n}$, such that the equations of $(2.6)$ are satisfied.

We can then set $Y_{i}(t) \triangleq \log X_{i}(t), 0 \leq t<\infty, i=1, \ldots n$, so that we have

$$
X_{i}(t)=\mathrm{e}^{Y_{i}(t)}, 0 \leq t<\infty, \quad i=1, \ldots n
$$

and can write the equations of (2.6) in the equivalent form

$$
\mathrm{d} Y_{i}(t)=\left(\frac{\mathrm{e}^{Y_{1}(t)}+\ldots+\mathrm{e}^{Y_{n}(t)}}{\mathrm{e}^{Y_{i}(t)}}\right)^{\beta} \mathrm{d} W_{i}(t), \quad Y_{i}(0)=\log X_{i}(0), \quad i=1, \ldots, n
$$

Thus the real-valued processes $\left(Y_{1}(\cdot), \ldots, Y_{n}(\cdot)\right)$ are continuous $\mathbf{F}$-local martingales with quadratic variations

$$
\left\langle Y_{i}\right\rangle(t)=\int_{0}^{t}\left(\frac{\mathrm{e}^{Y_{1}(s)}+\ldots+\mathrm{e}^{Y_{n}(s)}}{\mathrm{e}^{Y_{i}(s)}}\right)^{2 \beta} \mathrm{d} s=: A_{i}(t), \quad i=1, \ldots, n
$$

and $\left\langle Y_{i}, Y_{j}\right\rangle(t) \equiv 0$ for $i \neq j$.

The processes $t \mapsto A_{i}(t)$ are almost surely continuous and strictly increasing. Let us assume that $A_{i}(\infty)=\infty$, a.s. (we will return to this issue in a moment). Then we denote by $A_{i}^{-1}(\cdot)$ their inverses, and note that by F.B. Knight's theorem 
Chapter 2. System of SDEs for the Generalized Volatility-Stabilized Processes

(see for instance Theorem 4.13 in Karatzas and Shreve (1991)) the processes

$$
B_{i}(u) \triangleq Y_{i}\left(A_{i}^{-1}(u)\right), \quad 0 \leq u<\infty
$$

are independent Brownian motions with $B_{i}(0)=\log X_{i}(0)$ for $i=1, \ldots, n$, so we have the representations

$$
Y_{i}(t)=B_{i}\left(A_{i}(t)\right), \quad X_{i}(t)=\mathrm{e}^{B_{i}\left(A_{i}(t)\right)}, \quad 0 \leq t<\infty, i=1, \ldots, n .
$$

Substituting these expressions back into (2.9), we observe that the (continuous and strictly increasing) time-change processes $A_{1}(\cdot), \ldots, A_{n}(\cdot)$ satisfy the system of integral equations

$$
A_{i}(t)=\int_{0}^{t}\left(\frac{\mathrm{e}^{B_{1}\left(A_{1}(s)\right)}+\ldots+\mathrm{e}^{B_{n}\left(A_{n}(s)\right)}}{\mathrm{e}^{B_{i}\left(A_{i}(s)\right)}}\right)^{2 \beta} \mathrm{d} s, \quad 0 \leq t<\infty, i=1, \ldots, n .
$$

\section{Solvability of the system (2.11):}

Suppose that we are given $n$ independent Brownian motions $B_{1}(\cdot), \ldots, B_{n}(\cdot)$ with $B_{i}(0)=\log X_{i}(0)$, on the same probability space. We seek $n$ continuous, strictly increasing processes $A_{1}(\cdot), \ldots, A_{n}(\cdot)$ that satisfy $(2.11)$. These processes can be found by reducing the system of integral equations (2.11) to a single integral equation, in the following manner. First, we introduce the continuous, strictly increasing and real-valued processes

$$
Q_{i}(u) \triangleq \int_{0}^{u} \mathrm{e}^{2 \beta B_{i}(v)} \mathrm{d} v, \quad 0 \leq u<\infty
$$

with $Q_{i}(0)=0$, then we differentiate $(2.11)$ to rewrite it as

$$
\left(Q_{i}\left(A_{i}(t)\right)\right)^{\prime}=A_{i}^{\prime}(t) \mathrm{e}^{2 \beta B_{i}\left(A_{i}(t)\right)}=\left(\mathrm{e}^{B_{1}\left(A_{1}(t)\right)}+\ldots+\mathrm{e}^{B_{n}\left(A_{n}(t)\right)}\right)^{2 \beta},
$$


or equivalently as

$$
\begin{aligned}
Q_{i}\left(A_{i}(t)\right) & =\int_{0}^{t}\left(\mathrm{e}^{B_{1}\left(A_{1}(s)\right)}+\ldots+\mathrm{e}^{B_{n}\left(A_{n}(s)\right)}\right)^{2 \beta} \mathrm{d} s \\
& =\int_{0}^{t}\left(X_{1}(s)+\ldots+X_{n}(s)\right)^{2 \beta} \mathrm{d} s=: A(t)
\end{aligned}
$$

for $0 \leq t<\infty, i=1, \ldots, n$. Here, we have denoted the process

$$
A(t) \triangleq \int_{0}^{t}\left(\mathrm{e}^{B_{1}\left(A_{1}(s)\right)}+\ldots+\mathrm{e}^{B_{n}\left(A_{n}(s)\right)}\right)^{2 \beta} \mathrm{d} s, \quad 0 \leq t<\infty
$$

and observed that $\left(A^{\prime}(\cdot)\right)^{1 /(2 \beta)}=S(\cdot)=X_{1}(\cdot)+\ldots+X_{n}(\cdot)$. For each $i=1, \ldots, n$, the process $Q_{i}(\cdot)$ is adapted to the filtration $\mathbf{F}_{i}^{B}=\left\{\mathcal{F}_{i}^{B}(u)\right\}_{0 \leq u<\infty}$, where

$$
\mathcal{F}_{i}^{B}(u) \triangleq \sigma\left(B_{i}(v), 0 \leq v \leq u\right), \quad 0 \leq u<\infty .
$$

Moreover, with $B_{1}(\cdot), \ldots, B_{n}(\cdot)$ independent Brownian motions and with $B_{1}(0)=$ $\log X_{1}(0), \ldots, B_{n}(0)=\log X_{n}(0)$, the Engelbert-Schmidt zero-one laws (see for instance Karatzas and Shreve (1991), Proposition 6.27, p.216) guarantee that $0<$ $Q_{i}(\theta)<\infty$ for $0<\theta<\infty$, and $Q_{i}(\infty)=\infty$ hold a.s for each $i=1, \ldots, n$.

Let us denote by $P_{i}(\cdot)$ the inverses of the processes $Q_{i}(\cdot)$ in $(2.12)$; that is, we define

$$
P_{i}(\theta) \triangleq \inf \left\{u \geq 0: Q_{i}(u)>u\right\}, \quad 0 \leq \theta<\infty
$$

for each $i=1, \ldots, n$. We have clearly $P_{i}(0)=0, P_{i}(\infty)=\infty$. In terms of these strictly increasing processes $P_{i}(\cdot)$, the equations of (2.13) yield

$$
A_{i}(t)=P_{i}(A(t)), \quad 0 \leq t<\infty
$$

Next we define the continuous and strictly increasing process

$$
G(\theta) \triangleq\left(\sum_{j=1}^{n} \mathrm{e}^{B_{j}\left(P_{j}(\theta)\right)}\right)^{2 \beta}, \quad 0 \leq \theta<\infty
$$


so that, using (2.15) and recalling (2.10), we have

$$
\begin{aligned}
G(A(t)) & =\left(\sum_{j=1}^{n} \mathrm{e}^{B_{j}\left(P_{j}(A(t))\right)}\right)^{2 \beta}=\left(\sum_{j=1}^{n} \mathrm{e}^{B_{j}\left(A_{j}(t)\right)}\right)^{2 \beta}= \\
& =\left(\sum_{j=1}^{n} \mathrm{e}^{Y_{j}(t)}\right)^{2 \beta}=\left(\sum_{j=1}^{n} X_{j}(t)\right)^{2 \beta}, \quad 0 \leq t<\infty .
\end{aligned}
$$

Comparing (2.13) and (2.17), we obtain

$$
A(t)=\int_{0}^{t} G(A(s)) \mathrm{d} s, \quad 0 \leq t<\infty .
$$

In other words: the system of integral equations (2.11) has been reduced to the single integral equation (2.18). Once a solution to this integral equation has been found, the processes $A_{1}(\cdot), \ldots, A_{n}(\cdot)$ sought in the system $(2.11)$ are given by (2.15), in terms of the processes $P_{1}(\cdot), \ldots, P_{n}(\cdot)$ of $(2.14)$, which in turn are the inverses of the processes $Q_{1}(\cdot), \ldots, Q_{n}(\cdot)$ of $(2.12)$.

The integral equation (2.18) is solved by means of yet another time-change: namely, we introduce first the continuous and strictly increasing function

$$
\Upsilon(\theta) \triangleq \int_{0}^{\theta} \frac{1}{G(\xi)} \mathrm{d} \xi=\int_{0}^{\theta}\left(\sum_{j=1}^{n} \mathrm{e}^{B_{j}\left(P_{j}(\xi)\right)}\right)^{-2 \beta} \mathrm{d} \xi, \quad 0 \leq \theta<\infty
$$

and check, rather easily, that its inverse $A(\cdot)=\Upsilon^{-1}(\cdot)$, namely,

$$
A(t)=\inf \{\theta \geq 0: \Upsilon(\theta)>t\}, \quad 0 \leq t<\infty,
$$

solves the integral equation (2.18). Indeed, we have $A^{\prime}(t) \Upsilon^{\prime}(A(t))=1$, therefore

$$
A(t)=\int_{0}^{t} A^{\prime}(s) \mathrm{d} s=\int_{0}^{t} \frac{1}{\Upsilon^{\prime}(A(s))} \mathrm{d} s=\int_{0}^{t} G(A(s)) \mathrm{d} s, \quad 0 \leq t<\infty .
$$

Notice that this, in fact, is unique solution of the integral equation (2.18). Indeed, if $A(\cdot)$ is any continuous, strictly increasing process that satisfies (2.18) and $A(0)=0$, then it is necessarily the inverse of the process $\Upsilon(\cdot)$ in $(2.19)$. 
Chapter 2. System of SDEs for the Generalized Volatility-Stabilized

\section{Consolidation:}

Let us now recall the processes $P_{i}(\cdot)$ which were defined in $(2.14)$ as the inverses of the $\mathbf{F}_{i}^{B}$-adapted processes $Q_{i}(\cdot)$ in $(2.12)$, starting with the independent Brownian motions $B_{1}(\cdot), \ldots, B_{n}(\cdot)$. In particular, notice that if we set

$$
M_{i}(\theta) \triangleq B_{i}\left(P_{i}(\theta)\right), \quad 0 \leq \theta<\infty, i=1, \ldots, n
$$

then we have

$$
P_{i}(\theta)=\int_{0}^{\theta} \frac{1}{Q_{i}^{\prime}\left(P_{i}(\xi)\right)} \mathrm{d} \xi=\int_{0}^{\theta} \mathrm{e}^{-2 \beta B_{i}\left(P_{i}(\xi)\right)} \mathrm{d} \xi=\int_{0}^{\theta} \mathrm{e}^{-2 \beta M_{i}(\xi)} \mathrm{d} \xi
$$

Thus,

$$
M_{i}(\theta)=B_{i}\left(P_{i}(\theta)\right)=B_{i}\left(\int_{0}^{\theta} \mathrm{e}^{-2 \beta M_{i}(\xi)} \mathrm{d} \xi\right), \quad 0 \leq \theta<\infty,
$$

for $i=1, \ldots, n$, and

$$
\left\langle M_{i}\right\rangle(\theta)=P_{i}(\theta)=\int_{0}^{\theta} \mathrm{e}^{-2 \beta M_{i}(\xi)} \mathrm{d} \xi, \quad 0 \leq u<\infty
$$

It is clear that each $M_{i}(\cdot)$ in $(2.23)$ is a continuous local martingale relative to its own filtration $\mathbf{F}_{i}^{M}=\left\{\mathcal{F}_{i}^{M}(\theta)\right\}_{0 \leq \theta<\infty}$, where

$$
\mathcal{F}_{i}^{M}(\theta) \triangleq \sigma\left(M_{i}(\xi), 0 \leq \xi \leq \theta\right), \quad 0 \leq \theta<\infty
$$

and from (2.24) that it is also a diffusion process in natural scale, with dispersion coefficient $\sigma(m)=\mathrm{e}^{-\beta m}, m \in \mathbf{R}$. Therefore (using the fact that $M_{i}(0)=B_{i}(0)=$ $\left.Y_{i}(0)=\log X_{i}(0)\right)$ we deduce that $M_{i}(\cdot)$ satisfies the stochastic integral equation

$$
M_{i}(\theta)=\log X_{i}(0)+\int_{0}^{\theta} \mathrm{e}^{-\beta M_{i}(\xi)} \mathrm{d} V_{i}(\xi), \quad 0 \leq \theta<\infty
$$

where $V_{i}(\cdot) \triangleq \int_{0}^{\cdot} \mathrm{e}^{\beta M_{i}(\xi)} \mathrm{d} M_{i}(\xi)$ is an $\mathbf{F}_{i}^{M}$ - Brownian motion. We also know that the processes $M_{1}(\cdot), \ldots, M_{n}(\cdot)$ are independent; this is because for each $i=1, \ldots, n$ the process $M_{i}(\cdot)$ is $\mathcal{F}_{i}^{B}(\infty)$-measurable, and the Brownian motions $B_{1}(\cdot), \ldots, B_{n}(\cdot)$ are 
independent. Thus, the Brownian motions $V_{1}(\cdot), \ldots, V_{n}(\cdot)$ are independent as well.

Recalling the definition of $G(\cdot)$ in (2.16) and using the definition of processes $M_{i}(\cdot)$ in $(2.21)$, we can write

$$
G(\theta)=\left(\sum_{j=1}^{n} \mathrm{e}^{B_{j}\left(P_{j}(\theta)\right)}\right)^{2 \beta}=\left(\sum_{j=1}^{n} \mathrm{e}^{M_{j}(\theta)}\right)^{2 \beta}, \quad 0 \leq \theta<\infty,
$$

and notice that the process $G(\cdot)$ is adapted to the filtration

$$
\mathbf{F}^{M}=\left\{\mathcal{F}^{M}(\theta)\right\}_{0 \leq \theta<\infty} \text {, where } \mathcal{F}^{M}(\theta) \triangleq \sigma\left(M_{j}(\xi): 0 \leq \xi \leq \theta, j=1, \ldots, n\right)
$$

We recall also

$$
\Upsilon(\theta)=\int_{0}^{\theta} \frac{1}{G(\xi)} \mathrm{d} \xi=\int_{0}^{\theta}\left(\sum_{j=1}^{n} \mathrm{e}^{M_{j}(\xi)}\right)^{-2 \beta} \mathrm{d} \xi, \quad 0 \leq \theta<\infty
$$

of (2.19): a continuous, strictly increasing process which is $\mathbf{F}^{M}$-adapted, and realvalued. Indeed, $0<\Upsilon(u)<\infty$ for $0<u<\infty$, since

$$
0<\Upsilon(\theta)<\int_{0}^{\theta} \mathrm{e}^{-2 \beta M_{i}(\xi)} \mathrm{d} \xi=P_{i}(\theta)<\infty
$$

for $0<\theta<\infty$ and $i=1, \ldots, n$, where the inequality is a consequence of (2.28) and the equality comes from (2.22). In particular, we have $\Upsilon(0)=0$ and we will argue below, see (2.42), that

$$
\Upsilon(\infty)=\infty \quad \text { a.s. }
$$

Then we have $A(\infty)=\infty$, as well as $A_{i}(\infty)=P_{i}(A(\infty))=\infty$ for every $i=1, \ldots, n$, a.s.

The processes $G(\cdot)$ and $\Upsilon(\cdot)$ are both adapted to the filtration $\mathbf{F}^{M}$ of $(2.27)$. Thus, for the continuous, strictly increasing process $A(\cdot)=\Upsilon^{-1}(\cdot)$, the random 
variable $A(t)$ as in (2.20) is a stopping time of the filtration $\mathbf{F}^{M}$ for every $0 \leq t<\infty$; and by (2.7), (2.10), (2.15) and (2.21) we have

$$
\log X_{i}(t)=Y_{i}(t)=B_{i}\left(A_{i}(t)\right)=B_{i}\left(P_{i}(A(t))=M_{i}(A(t)), \quad 0 \leq t<\infty,\right.
$$

therefore each $X_{i}(\cdot)$ is $\mathcal{F}^{M}(\infty)$-measurable. We deduce from all this

$$
X_{i}(t)=\mathrm{e}^{Y_{i}(t)}, \text { where } Y_{i}(t)=M_{i}(A(t)), \quad 0 \leq t<\infty
$$

for $i=1, \ldots, n$, and each $X_{i}(\cdot)$ is $\mathcal{F}^{M}(\infty)$-measurable.

In other words, given the paths of the vector-valued process $M(\cdot)=\left(M_{1}(\cdot)\right.$, $\left.\ldots, M_{n}(\cdot)\right)$, the components of which are independent one-dimensional diffusions in natural scale, the paths of the vector process $X(\cdot)=\left(X_{1}(\cdot), \ldots, X_{n}(\cdot)\right)$ are determined uniquely. In particular, the distribution of $X(\cdot)$ is determined uniquely from the distribution of $M(\cdot)$.

\subsubsection{Synthesis}

On a filtered probability space $(\Omega, \mathcal{F}, \mathbb{P}), \mathbf{F}=\{\mathcal{F}(t)\}_{0 \leq t<\infty}$ rich enough to carry $n$ independent Brownian motions $V_{1}(\cdot), \ldots, V_{n}(\cdot)$, we consider independent diffusions in natural scale as in (2.25), satisfying the stochastic differential equations

$$
\mathrm{d} M_{i}(\theta)=\mathrm{e}^{-\beta M_{i}(\theta)} \mathrm{d} V_{i}(\theta), \quad M_{i}(0)=\log X_{i}(0)
$$

These equations can be solved in the weak sense by the method of time-change (see Karatzas and Shreve (1991), Theorem 5.4 and Remark 5.5, p.332), and the dispersion coefficient $\sigma(m)=\mathrm{e}^{-\beta m}$ is locally Lipschitz continuous in $m \in \mathbb{R}$. In other words, pathwise uniqueness holds for these equations, and this implies the equations are strongly solvable:

$$
\mathcal{F}_{i}^{M}(\theta)=\mathcal{F}_{i}^{V}(\theta), \quad 0 \leq \theta<\infty, i=1, \ldots, n
$$


where we have defined the $\sigma$-algebras $\mathcal{F}_{i}^{M}(\theta) \triangleq \sigma\left(M_{i}(\xi), 0 \leq \xi \leq \theta\right)$ and $\mathcal{F}_{i}^{V}(\theta) \triangleq$ $\sigma\left(V_{i}(\xi), 0 \leq \xi \leq \theta\right)$ for every $0 \leq \theta<\infty$ and $i=1, \ldots, n$.

The resulting diffusion processes are also local martingales in their own filtrations $\mathbf{F}_{i}^{M}=\mathbf{F}_{i}^{V}$, thus also in the larger filtration $\mathbf{F}^{M}=\mathbf{F}^{V}$, with quadratic variations

$$
\left\langle M_{i}\right\rangle(\theta)=\int_{0}^{\theta} \mathrm{e}^{-2 \beta M_{i}(\xi)} \mathrm{d} \xi, \quad 0 \leq \theta<\infty, i=1, \ldots, n
$$

and $\left\langle M_{i}, M_{j}\right\rangle(t) \equiv 0$ for $i \neq j$. Let us denote these continuous and strictly increasing variations by $P_{i}(\cdot)$ and their inverses by $Q_{i}(\cdot)$, that is,

$$
\begin{array}{cc}
P_{i}(\theta) \triangleq\left\langle M_{i}\right\rangle(\theta)=\int_{0}^{\theta} \mathrm{e}^{-2 \beta M_{i}(\xi)} \mathrm{d} \xi, & 0 \leq \theta<\infty, i=1, \ldots, n, \\
Q_{i}(u) \triangleq \inf \left\{\theta \geq 0: P_{i}(\theta)>u\right\}, & 0 \leq u<\infty, i=1, \ldots, n
\end{array}
$$

Then by F.B. Knight's theorem (see for instance Theorem 4.13 in Karatzas and Shreve (1991)) the processes

$$
B_{i}(u) \triangleq M_{i}\left(Q_{i}(u)\right), \quad 0 \leq u<\infty, i=1, \ldots, n
$$

are independent, standard Brownian motions, and we have the representations

$$
M_{i}(\theta)=B_{i}\left(P_{i}(\theta)\right), \quad 0 \leq \theta<\infty, i=1, \ldots, n
$$

Furthermore, we have for each $i=1, \ldots, n$ the representation

$$
Q_{i}(u)=\int_{0}^{u} \frac{1}{P_{i}^{\prime}\left(Q_{i}(v)\right)} \mathrm{d} v=\int_{0}^{u} \mathrm{e}^{2 \beta B_{i}(v)} \mathrm{d} v, \quad 0 \leq u<\infty
$$

in accordance with $(2.12),(2.14)$ and $(2.22)$. Notice also that not only is $B_{i}(\cdot)$ measurable with respect to $\mathcal{F}_{i}^{M}(\infty)$, but clearly each $M_{i}(\cdot)$ is $\mathcal{F}_{i}^{B}(\infty)$-measurable thanks to the representations $(2.31)$ and the fact that the process $P_{i}(\cdot)$ is the inverse of the $\mathbf{F}_{i}^{B}$-adapted process $Q_{i}(\cdot)$, for each $i, \ldots, n$. Therefore $\mathcal{F}_{i}^{B}(\infty)=\mathcal{F}_{i}^{M}(\infty)$, that is, 
Chapter 2. System of SDEs for the Generalized Volatility-Stabilized

each $M_{i}(\cdot), i=1, \ldots, n$ is a pure martingale in the sense of Dubins and Schwarz (1967).

We now introduce the $\mathbf{F}^{M}$-adapted processes

$$
G(\theta):=\left(\sum_{j=1}^{n} \mathrm{e}^{M_{j}(\theta)}\right)^{2 \beta}, \quad \Upsilon(\theta):=\int_{0}^{\theta} \frac{1}{G(\xi)} \mathrm{d} \xi ; \quad 0 \leq \theta<\infty
$$

in accordance with (2.26), (2.28), as well as the $\mathbf{F}^{M}$-stopping times

$$
A(t):=\inf \{\theta \geq 0: \Upsilon(\theta)>t\}
$$

for every $0 \leq t<\infty$, in accordance with (2.20). Note that

$$
A(t)=\int_{0}^{t} \frac{1}{\Upsilon^{\prime}(A(s))} \mathrm{d} s=\int_{0}^{t} G(A(s)) \mathrm{d} s
$$

as in (2.18), so that $A(\cdot)$ is adapted to the time-changed filtration

$$
\mathbf{G}=\{\mathcal{G}(t)\}_{0 \leq t<\infty}, \quad \text { where } \mathcal{G}(t) \triangleq \mathcal{F}^{M}(A(t)), \quad 0 \leq t<\infty .
$$

Now, for each $i=1, \ldots, n$ consider $\mathbf{G}$-adapted processes

$$
Y_{i}(t) \triangleq M_{i}(A(t)), \quad X_{i}(t) \triangleq \mathrm{e}^{Y_{i}(t)}=\mathrm{e}^{M_{i}(A(t))}, \quad 0 \leq t<\infty,
$$

and

$$
\begin{aligned}
W_{i}(t) & \triangleq \int_{0}^{A(t)} \frac{1}{\sqrt{G(\theta)}} \mathrm{d} V_{i}(\theta)=\int_{0}^{A(t)}\left(\frac{\mathrm{e}^{M_{i}(\theta)}}{\mathrm{e}^{M_{1}(\theta)}+\cdots+\mathrm{e}^{M_{n}(\theta)}}\right)^{\beta} \mathrm{d} M_{i}(\theta) \\
& =\int_{0}^{t}\left(\frac{\mathrm{e}^{Y_{i}(s)}}{\mathrm{e}^{Y_{1}(s)}+\cdots+\mathrm{e}^{Y_{n}(s)}}\right)^{\beta} \mathrm{d} Y_{i}(s) \\
& =\int_{0}^{t}\left(\frac{X_{i}(s)}{X_{1}(s)+\cdots+X_{n}(s)}\right)^{\beta} \mathrm{d} \log X_{i}(s), \quad 0 \leq t<\infty
\end{aligned}
$$

in accordance with (2.30), (2.25), (2.8) and (2.6). All these processes are clearly continuous G-local martingales, and we have

$$
\left\langle W_{i}, W_{j}\right\rangle(t)=\delta_{i j} \int_{0}^{A(t)} \frac{1}{G(\theta)} \mathrm{d} \theta=\delta_{i j} \Upsilon(A(t))=\delta_{i j} t .
$$


Chapter 2. System of SDEs for the Generalized Volatility-Stabilized

From P. Lévy's theorem (see for instance Theorem 3.16 in Karatzas and Shreve (1991)), we deduce that $W_{1}(\cdot), \ldots, W_{n}(\cdot)$ are independent Brownian motions, adapted both to $\mathbf{G}$ and to the smaller filtration $\mathbf{F}=\{\mathcal{F}(t)\}_{0 \leq t<\infty}$ with

$$
\mathcal{F}(t) \triangleq \mathcal{F}^{X}(t)=\sigma\left(X_{j}(s): 0 \leq s \leq t, j=1, \ldots, n\right), \quad 0 \leq t<\infty
$$

It follows from $(2.34)$ that the processes $X(\cdot), W(\cdot)$ of $(2.33),(2.34)$ solve on the filtered probability space $(\Omega, \mathcal{F}, \mathbb{P}), \mathbf{F}$ the system of equations $(2.6)$.

We have constructed a weak solution, and shown that this solution is unique in the sense of the probability law (as is discussed in the end of the "Consolidation" part of the Analysis section).

\subsubsection{Lamperti-Jacobsen-type representations}

From the stochastic differential equations (2.25) it is not difficult to check that the processes

$$
N_{i}(\theta) \triangleq \mathrm{e}^{M_{i}(\theta)}, \quad 0 \leq \theta<\infty
$$

are also independent, one-dimensional diffusions with dynamics

$$
\mathrm{d} N_{i}(\theta)=\frac{1}{2}\left(N_{i}(\theta)\right)^{1-2 \beta} \mathrm{d} \theta+\left(N_{i}(\theta)\right)^{1-\beta} \mathrm{d} V_{i}(\theta), \quad 0 \leq \theta<\infty
$$

for $i=1, \ldots, n$. In this new notation, we can re-write (2.30) and (2.26) as

$$
X_{i}(t)=N_{i}(A(t)), \quad G(\theta)=\left(\sum_{j=1}^{n} N_{j}(\theta)\right)^{2 \beta}
$$

which together with (2.17) and (2.18) leads to the Lamperti-Jacobsen-type Representation

$$
X_{i}(t)=N_{i}\left(\int_{0}^{t}\left(\sum_{j=1}^{n} X_{j}(s)\right)^{2 \beta} \mathrm{d} s\right), \quad 0 \leq t<\infty, i=1, \ldots, n
$$


for the solution $X_{1}(\cdot), \ldots, X_{n}(\cdot)$ of the system $(2.6)$ in terms of the diffusions in (2.36). On the other hand, we also have $N_{i}(\theta)=X_{i}(\Upsilon(\theta))$, where $\Upsilon(\cdot)=A^{-1}(\cdot)$ is given by $(2.19)$ as

$$
\Upsilon(\theta)=\int_{0}^{\theta}(G(\xi))^{-1} \mathrm{~d} \xi=\int_{0}^{\theta}\left(\sum_{j=1}^{n} N_{j}(\xi)\right)^{-2 \beta} \mathrm{d} \xi
$$

This leads to the Dual Representation

$$
N_{i}(\theta)=X_{i}\left(\int_{0}^{\theta}\left(\sum_{j=1}^{n} N_{j}(\xi)\right)^{-2 \beta} \mathrm{d} \xi\right), \quad 0 \leq \theta<\infty, i=1, \ldots, n
$$

for the diffusions in $(2.36)$, in terms of the processes $X_{1}(\cdot), \ldots, X_{n}(\cdot)$ in $(2.36)$.

\subsubsection{Connection to Bessel and Squared-Bessel processes}

For a brief introduction to Bessel and square-Bessel processes we refer the reader to the Appendix which contains the definitions as well as a few known results regarding the properties of these processes.

It can be easily checked from (2.36) that

$$
Z_{i}(\theta) \triangleq \frac{1}{\beta^{2}}\left(N_{i}(\theta)\right)^{2 \beta}, \quad 0 \leq \theta<\infty
$$

are independent 2-dimensional squared-Bessel processes:

$$
\mathrm{d} Z_{i}(\theta)=2 \mathrm{~d} \theta+2 \sqrt{Z_{i}(\theta)} \mathrm{d} V_{i}(\theta), \quad 0 \leq \theta<\infty, i=1, \ldots, n
$$

Similarly, it can be checked from (2.36) that

$$
R_{i}(\theta) \triangleq \frac{1}{\beta}\left(N_{i}(\theta)\right)^{\beta}, \quad 0 \leq \theta<\infty
$$

are independent 2-dimensional Bessel processes:

$$
\mathrm{d} R_{i}(\theta)=\frac{\mathrm{d} \theta}{2 R_{i}(\theta)}+\mathrm{d} V_{i}(\theta), \quad 0 \leq \theta<\infty, i=1, \ldots, n .
$$


In terms of the independent squared-Bessel processes in (2.39) (resp., the independent Bessel processes in (2.40)), we have the representations

$$
X_{i}(t)=\left(\beta^{2} Z_{i}(A(t))\right)^{\frac{1}{2 \beta}}=\left(\beta R_{i}(A(t))\right)^{\frac{1}{\beta}}, \quad 0 \leq t<\infty
$$

as well as the corresponding Lamperti-Jacobsen-type representations

$$
X_{i}(t)=\left(\beta R_{i}\left(\int_{0}^{t}\left(\sum_{j=1}^{n} X_{j}(s)\right)^{2 \beta} \mathrm{d} s\right)\right)^{\frac{1}{\beta}}, \quad 0 \leq t<\infty
$$

for the processes $X_{i}(\cdot), i=1, \ldots, n$. Here $A(\cdot)$ is the inverse of the continuous, strictly increasing process $\Upsilon(\cdot)$ of $(2.28)$, now written in the form

$$
\Upsilon(\theta)=\frac{1}{\beta^{2}} \int_{0}^{\theta}\left(\sum_{j=1}^{n}\left(Z_{j}(\xi)\right)^{1 /(2 \beta)}\right)^{-2 \beta} \mathrm{d} \xi, \quad 0 \leq \theta<\infty .
$$

From this representation, it is now possible to deduce $\Upsilon(\infty)=\infty$ a.s. for any value of $\beta \in(0, \infty)$, as follows. We have

$$
\begin{aligned}
\beta^{2} \Upsilon(\theta) & \geq \int_{0}^{\theta}\left(n \max _{1 \leq j \leq n}\left\{\left(Z_{j}(\xi)\right)^{1 /(2 \beta)}\right\}\right)^{-2 \beta} \mathrm{d} \xi=\int_{0}^{\theta} n^{-2 \beta}\left(\max _{1 \leq j \leq n}\left\{Z_{j}(\xi)\right\}\right)^{-1} \mathrm{~d} \xi \\
& \geq n^{-2 \beta} \int_{0}^{\theta}\left(\sum_{j=1}^{n} Z_{j}(\xi)\right)^{-1} \mathrm{~d} \xi=n^{-2 \beta} \int_{0}^{\theta} \frac{1}{Z(\xi)} \mathrm{d} \xi .
\end{aligned}
$$

Here

$$
Z(\xi) \triangleq \sum_{j=1}^{n} Z_{j}(\xi), \quad 0 \leq \xi<\infty
$$

is a squared-Bessel process in dimension $2 n$, by the additivity property of independent squared-Bessel processes, (see Revuz and Yor (1999), (1.2) Theorem). But then Lemma 4.2 in Cherny (2000) shows that

$$
\lim _{\theta \rightarrow \infty} \frac{1}{\log (\theta)} \int_{0}^{\theta} \frac{1}{Z(\xi)} \mathrm{d} \xi=\frac{1}{2(n-1)}
$$

holds a.s. This, in conjunction with (2.42), implies that for any value of $\beta \in(0, \infty)$ we have $\Upsilon(\infty)=\infty$ a.s., thus also $A(\infty)=\infty$ a.s. 
In fact, one can describe the asymptotic behavior of the process $\Upsilon(\cdot)$, namely, we have the asymptotic property (4.6).

Remark: As was noted in Goia (2009), the volatility-stabilized processes exhibit some similarity with the classical Black-Scholes market model which consists of $n$ stocks with capitalizations following the geometric Brownian motions dynamics. The Lamperti representation implies that the stock capitalizations in Black-Scholes model can be represented as time-changed squared-Bessel processes, with the time change being intrinsic to each stock. On the contrary, in the volatility-stabilized markets, as well as in the generalized volatility-stabilized markets introduced here, the time change process depends on the entire market but is the same for each stock.

\subsubsection{Solution of the SDEs - The General Case}

Since we already described how to construct a solution in the simple case in the previous subsection, we can now make use of those observations in the general case with $\alpha_{1} \geq 0, \ldots, \alpha_{n} \geq 0, \sigma>0, \beta>0$, and a given measurable function $K(\cdot):(0, \infty)^{n} \rightarrow(0, \infty)$.

\subsubsection{Analysis}

Suppose we have constructed a weak solution of the system (2.4); in other words, suppose that on some filtered probability space $(\Omega, \mathcal{F}, \mathbb{P}), \mathbf{F}=\{\mathcal{F}(t)\}_{0 \leq t<\infty}$ we have constructed independent Brownian motions $\left(W_{1}(\cdot), \ldots, W_{n}(\cdot)\right)$ and continuous, strictly positive and adapted processes $\left(X_{1}(\cdot), \ldots, X_{n}(\cdot)\right)$, such that the integral version of (2.4) is satisfied, namely, for $i=1, \ldots n$, and $0 \leq t<\infty$

$$
X_{i}(t)=x_{i}+\frac{\alpha_{i}+\sigma^{2}}{2} \int_{0}^{t} \frac{[\mathcal{T}(X(s))]^{2}}{\left(X_{i}(s)\right)^{2 \beta-1}} \mathrm{~d} s+\sigma \int_{0}^{t} \frac{\mathcal{T}(X(s))}{\left(X_{i}(s)\right)^{\beta-1}} \mathrm{~d} W_{i}(s)
$$


with the function $\mathcal{T}(\cdot):(0, \infty)^{n} \rightarrow(0, \infty)$ defined in $(2.3)$, namely

$$
\mathcal{T}(x)=\left(\sum_{i=1}^{n} x_{i}\right)^{\beta} K(x), \quad x \in(0, \infty)^{n} .
$$

Consider the continuous, strictly increasing process $A(\cdot)$ defined as follows

$$
A(t) \triangleq \int_{0}^{t}[\mathcal{T}(X(s))]^{2} \mathrm{~d} s, \quad 0 \leq t<\infty .
$$

This process $A(\cdot)$ is clearly adapted to the filtration $\mathbf{F}^{X}=\left\{\mathcal{F}^{X}(t)\right\}_{0 \leq t<\infty}$, where

$$
\mathcal{F}^{X}(t) \triangleq \sigma\left(X_{i}(s): 0 \leq s \leq t, i=1, \ldots, n\right), \quad 0 \leq t<\infty .
$$

We have $A(0)=0$, and assume that

$$
A(t)<\infty, t \in(0, \infty) \text {, and } A(\infty)=\infty \text { a.s. }{ }^{1}
$$

Let us also denote by

$$
\Upsilon(\theta) \triangleq \inf \{t \geq 0: A(t)>\theta\}, \quad 0 \leq \theta<\infty
$$

the inverse of this increasing process, and note that each $\Upsilon(\theta)$ is an $\mathbf{F}^{X}$-stopping time. Therefore,

$$
\mathbf{H}=\{\mathcal{H}(\theta)\}_{0 \leq \theta<\infty}, \quad \text { where } \mathcal{H}(\theta) \triangleq \mathcal{F}^{X}(\Upsilon(\theta)), 0 \leq \theta<\infty
$$

defines another filtration on this space. Note also that, if we define

$$
\begin{aligned}
& N_{i}(\theta) \triangleq X_{i}(\Upsilon(\theta)), \quad 0 \leq \theta<\infty, i=1, \ldots, n \\
& N(\theta)=\left(N_{1}(\theta), \ldots, N_{n}(\theta)\right)=X(\Upsilon(\theta)), \quad 0 \leq \theta<\infty
\end{aligned}
$$

and

$$
G(\theta) \triangleq[\mathcal{T}(N(\theta))]^{2}, \quad 0 \leq \theta<\infty,
$$

we have by virtue of $A(\Upsilon(\theta))=\theta$ the representation $A(\cdot)=\int_{0}^{\cdot} G(A(t)) \mathrm{d} t$, as well as

$$
\Upsilon(\theta)=\int_{0}^{\theta} \frac{1}{A^{\prime}(\Upsilon(\xi))} \mathrm{d} \xi=\int_{0}^{\theta} \frac{1}{[\mathcal{T}(X(\Upsilon(\xi)))]^{2}} \mathrm{~d} \xi
$$


Chapter 2. System of SDEs for the Generalized Volatility-Stabilized

$$
=\int_{0}^{\theta} \frac{1}{[\mathcal{T}(N(\xi))]^{2}} d \xi=\int_{0}^{\theta} \frac{1}{G(\xi)} d \xi .
$$

In particular, with $\mathbf{F}^{N}=\left\{\mathcal{F}^{N}(\theta)\right\}_{0 \leq \theta<\infty}$ where

$$
\mathcal{F}^{N}(\theta) \triangleq \sigma\left(N_{j}(\xi): 0 \leq \xi \leq \theta, j=1, \ldots, n\right), \quad 0 \leq \theta<\infty,
$$

we see that the processes $G(\cdot), \Upsilon(\cdot)$ are $\mathbf{F}^{N}$-adapted.

Consider now for $i=1, \ldots, n$ the continuous local martingales

$$
V_{i}(\theta) \triangleq \int_{0}^{\Upsilon(\theta)} \sqrt{A^{\prime}(t)} \mathrm{d} W_{i}(t)=\int_{0}^{\Upsilon(\theta)} \mathcal{T}(X(t)) \mathrm{d} W_{i}(t), \quad 0 \leq \theta<\infty,
$$

of the filtration $\mathbf{H}$ in (2.46). They satisfy

$$
\left\langle V_{i}, V_{j}\right\rangle(\theta)=\delta_{i j} \int_{0}^{\Upsilon(\theta)} A^{\prime}(t) \mathrm{d} t=\delta_{i j} A(\Upsilon(\theta))=\delta_{i j} \theta,
$$

so $V_{1}(\cdot), \ldots, V_{n}(\cdot)$ are independent Brownian motions by the P. Lévy theorem (see for instance Theorem 3.16 in Karatzas and Shreve (1991)). In terms of these processes, and in conjunction with the consequence

$$
X_{i}(t)=N_{i}(A(t)), \quad 0 \leq t<\infty, i=1, \ldots, n
$$

of (2.47), we may rewrite the system of equations (2.4) as

$$
\begin{aligned}
\mathrm{d} X_{i}(t) & =\frac{\alpha_{i}+\sigma^{2}}{2\left(X_{i}(t)\right)^{2 \beta-1}} \mathrm{~d} A(t)+\frac{\sigma}{\left(X_{i}(t)\right)^{\beta-1}} \mathrm{~d} V_{i}(A(t)) \\
& =\frac{\alpha_{i}+\sigma^{2}}{2\left(N_{i}(A(t))\right)^{2 \beta-1}} \mathrm{~d} A(t)+\frac{\sigma}{\left(N_{i}(A(t))\right)^{\beta-1}} \mathrm{~d} V_{i}(A(t)),
\end{aligned}
$$

or equivalently as

$$
N_{i}(A(t))=X_{i}(t)=x_{i}+\frac{\alpha_{i}+\sigma^{2}}{2} \int_{0}^{A(t)} \frac{1}{\left(N_{i}(\xi)\right)^{2 \beta-1}} \mathrm{~d} \xi+\sigma \int_{0}^{A(t)} \frac{1}{\left(N_{i}(\xi)\right)^{\beta-1}} \mathrm{~d} V_{i}(\xi) .
$$

This leads us to the system of stochastic differential equations

$$
\mathrm{d} N_{i}(\theta)=\frac{\alpha_{i}+\sigma^{2}}{2}\left(N_{i}(\theta)\right)^{1-2 \beta} \mathrm{d} \theta+\sigma\left(N_{i}(\theta)\right)^{1-\beta} \mathrm{d} V_{i}(\theta),
$$


Chapter 2. System of SDEs for the Generalized Volatility-Stabilized

Processes

$$
N_{i}(0)=x_{i} \in(0, \infty), \quad i=1, \ldots, n
$$

for the processes of (2.47).

Next, we define

$$
Z_{i}(\theta) \triangleq \frac{1}{(\beta \sigma)^{2}}\left(N_{i}(\theta)\right)^{2 \beta}, \quad 0 \leq \theta<\infty,
$$

and note from (2.52) that this process satisfies the stochastic differential equation

$$
\begin{aligned}
& \mathrm{d} Z_{i}(\theta)=m_{i} \mathrm{~d} \theta+2 \sqrt{Z_{i}(\theta)} \mathrm{d} V_{i}(\theta), \quad 0 \leq \theta<\infty \\
& Z_{i}(0)=\frac{1}{(\beta \sigma)^{2}} x_{i}^{2 \beta}=: z_{i}>0
\end{aligned}
$$

for a squared-Bessel process in "dimension" $m_{i} \triangleq 2+\alpha_{i} /\left(\beta \sigma^{2}\right) \geq 2$, for each $i=1, \ldots, n$.

Remark: Similarly, we can also define

$$
R_{i}(\theta) \triangleq \frac{1}{\beta \sigma}\left(N_{i}(\theta)\right)^{\beta}, \quad 0 \leq \theta<\infty,
$$

by analogy with (2.40), and note that this process satisfies the stochastic differential equation

$$
\begin{aligned}
& \mathrm{d} R_{i}(\theta)=\frac{m_{i}-1}{2 R_{i}(\theta)} \mathrm{d} \theta+\mathrm{d} V_{i}(\theta), \quad 0 \leq \theta<\infty \\
& R_{i}(0)=\frac{1}{\beta \sigma} x_{i}^{\beta}=: r_{i}>0
\end{aligned}
$$

for a Bessel process in "dimension" $m_{i}=2+\alpha_{i} /\left(\beta \sigma^{2}\right) \geq 2$, for each $i=1, \ldots, n$.

Since the squared-Bessel SDE of (2.54) with dimension $m_{i} \geq 2$ admits a pathwise unique, strong and strictly positive solution, we have

$$
\mathcal{F}_{i}^{N}(\theta)=\mathcal{F}_{i}^{Z}(\theta)=\mathcal{F}_{i}^{V}(\theta), \quad 0 \leq \theta<\infty, i=1, \ldots, n
$$

where we have defined the filtrations $\mathcal{F}_{i}^{N}(\theta) \triangleq \sigma\left(N_{i}(\xi): 0 \leq \xi \leq \theta\right), \mathcal{F}_{i}^{Z}(\theta) \triangleq$ $\sigma\left(Z_{i}(\xi): 0 \leq \xi \leq \theta\right)$, and $\mathcal{F}_{i}^{V}(\theta) \triangleq \sigma\left(V_{i}(\xi): 0 \leq \xi \leq \theta\right)$ for every $0 \leq \theta<\infty$ 
and $i=1, \ldots, n$. Since the processes $V_{1}(\cdot), \ldots, V_{n}(\cdot)$ are independent, (2.57) implies that the squared-Bessel processes $Z_{1}(\cdot), \ldots, Z_{n}(\cdot)$ of $(2.53)$ are also independent; and thus so are the processes $N_{1}(\cdot), \ldots, N_{n}(\cdot)$ of $(2.52)$.

It follows also from (2.48), (2.50) and (2.53) that the inverse of the timechange $A(\cdot)$ of $(2.43)$ is given as

$$
\begin{aligned}
\Upsilon(\theta) & =\inf \{t \geq 0 ; A(t)>\theta\}=\int_{0}^{\theta}[\mathcal{T}(N(\xi))]^{-2} \mathrm{~d} \xi \\
& =\int_{0}^{\theta}\left[\mathcal{T}\left((\beta \sigma)^{\frac{1}{\beta}}\left(Z_{1}(\xi)\right)^{\frac{1}{2 \beta}}, \ldots,(\beta \sigma)^{\frac{1}{\beta}}\left(Z_{n}(\xi)\right)^{\frac{1}{2 \beta}}\right)\right]^{-2} \mathrm{~d} \xi
\end{aligned}
$$

for any $0 \leq \theta<\infty$. Now it is clear, recalling (2.50) and (2.53) once more, that the processes

$$
X_{i}(t)=N_{i}(A(t))=(\beta \sigma)^{\frac{1}{\beta}}\left(Z_{i}(A(t))\right)^{\frac{1}{2 \beta}}, \quad 0 \leq t<\infty
$$

for $i=1, \ldots, n$, are all $\mathcal{F}^{Z}(\infty)$-measurable, since the process $A(\cdot)$ is the inverse of the $\mathbf{F}^{Z}$-adapted process $\Upsilon(\cdot)$ in (2.58).

In conclusion, we see that, if (2.44) is satisfied and if the vector processes $X(\cdot)$ and $W(\cdot)$ are parts of a weak solution of the equation (2.1) or (2.4), then $X(\cdot)$ is necessarily of the form (2.59), expressible in terms of some appropriate independent squared-Bessel processes $Z_{1}(\cdot), \ldots, Z_{n}(\cdot)$ as in $(2.54)$, in dimensions $m_{1}, \ldots, m_{n}$, respectively. In particular, since the paths of $\left(X_{1}(\cdot), \ldots, X_{n}(\cdot)\right)$ are determined uniquely from the paths of $\left(Z_{1}(\cdot), \ldots, Z_{n}(\cdot)\right)$, the joint distributions of $\left(X_{1}(\cdot), \ldots, X_{n}(\cdot)\right)$ are determined uniquely. In other words, uniqueness in distribution holds for the system of equations (2.1), as well as for the system of equations $(2.4)$.

Remark: For a specific choice of the function $K(\cdot)$, and the corresponding function $\mathcal{T}(\cdot)$ as in (2.3), one can use the representation in (2.58) and the properties of squared Bessel processes to decide whether this choice of $K(\cdot)$ implies $\Upsilon(\theta)<\infty, \theta \in(0, \infty)$, 
Chapter 2. System of SDEs for the Generalized Volatility-Stabilized Processes

and $\Upsilon(\infty)=\infty$ a.s. (thus (2.44) also holds). Examples of sufficient conditions on $K(\cdot)$ for these to be satisfied are discussed in Section 2.3.

\subsubsection{Synthesis}

Let us follow now this same thread in reverse, in an effort actually to construct a weak solution to the system of $(2.4)$. On a filtered probability space $(\Omega, \mathcal{F}, \mathbb{P})$, $\mathbf{F}=\{\mathcal{F}(t)\}_{0 \leq t<\infty}$ rich enough to carry $n$ independent standard Brownian motions $V_{1}(\cdot), \ldots, V_{n}(\cdot)$, we construct the squared-Bessel processes described by stochastic equations of the form

$$
\mathrm{d} Z_{i}(\theta)=m_{i} \mathrm{~d} \theta+2 \sqrt{Z_{i}(\theta)} \mathrm{d} V_{i}(\theta), \quad Z_{i}(0)=\frac{1}{(\beta \sigma)^{2}} x_{i}^{2 \beta}>0
$$

with $m_{i}=2+\alpha_{i} /\left(\beta \sigma^{2}\right) \geq 2$ for $i=1, \ldots, n$ as in (2.54). These equations admit pathwise unique, strong and strictly positive solutions, so

$$
\mathcal{F}_{i}^{Z}(\theta)=\mathcal{F}_{i}^{V}(\theta), \quad 0 \leq \theta<\infty, i=1, \ldots, n
$$

where $\mathcal{F}_{i}^{Z}(\theta) \triangleq \sigma\left(Z_{i}(\xi): 0 \leq \xi \leq \theta\right)$ and $\mathcal{F}_{i}^{V}(\theta) \triangleq \sigma\left(V_{i}(\xi): 0 \leq \xi \leq \theta\right)$. Let us also denote $\mathbf{F}^{Z}=\left\{\mathcal{F}^{Z}(\theta)\right\}_{0 \leq \theta<\infty}$, where

$$
\mathcal{F}^{Z}(\theta) \triangleq \sigma\left(Z_{i}(\xi): 0 \leq \xi \leq \theta, i=1, \ldots, n\right), \quad 0 \leq \theta<\infty
$$

In terms of the squared-Bessel processes $Z(\cdot)=\left(Z_{1}(\cdot), \ldots, Z_{n}(\cdot)\right)$ and by analogy with (2.58), we define then the continuous, strictly increasing and $\mathbf{F}^{Z}$-adapted time change process

$$
\Upsilon(\theta) \triangleq \int_{0}^{\theta}\left[\mathcal{T}\left((\beta \sigma)^{\frac{1}{\beta}}\left(Z_{1}(\xi)\right)^{\frac{1}{2 \beta}}, \ldots,(\beta \sigma)^{\frac{1}{\beta}}\left(Z_{n}(\xi)\right)^{\frac{1}{2 \beta}}\right)\right]^{-2} \mathrm{~d} \xi
$$

for $0 \leq \theta<\infty$, as in (2.58). The function $\mathcal{T}$ is defined in (2.3). Obviously we have $\Upsilon(0)=0$ a.s. 
Let us now assume that the process $\Upsilon(\cdot)$ satisfies the following two properties $^{2}$ :

$$
\begin{array}{ll}
(P 1) & \Upsilon(\theta)<\infty \text { a.s., for } \theta \in(0, \infty) \\
(P 2) & \lim _{\theta \rightarrow \infty} \Upsilon(\theta)=\infty \text { a.s. }
\end{array}
$$

Next, we define the process $A(\cdot)$ as the inverse of $\Upsilon(\cdot)$, that is

$$
A(t) \triangleq \inf \{\theta \geq 0: \Upsilon(\theta)>t\}, \quad 0 \leq t<\infty
$$

and note that the process $A(\cdot)$ is strictly increasing, continuous and satisfies $A(0)=$ $0, A(t)<\infty, t \in(0, \infty)$, and $A(\infty)=\infty$ a.s.

Moreover, each $A(t)$ is a stopping time of the filtration $\mathbf{F}^{Z}$, therefore

$$
\mathbf{G}=\{\mathcal{G}(\theta)\}_{0 \leq \theta<\infty}, \quad \text { where } \mathcal{G}(\theta) \triangleq \mathcal{F}^{Z}(A(\theta)), 0 \leq \theta<\infty
$$

is also a filtration. The processes

$$
\begin{aligned}
& N_{i}(\theta) \triangleq(\beta \sigma)^{\frac{1}{\beta}}\left(Z_{i}(\theta)\right)^{\frac{1}{2 \beta}}, \quad 0 \leq \theta<\infty \\
& X_{i}(t) \triangleq N_{i}(A(t)), \quad 0 \leq t<\infty
\end{aligned}
$$

defined for $i=1, \ldots, n$ according to (2.53) and (2.50), are respectively $\mathbf{F}^{Z}$-adapted and G-adapted. Furthermore, $X(\cdot)=\left(X_{1}(\cdot), \ldots, X_{n}(\cdot)\right)$ is $\mathcal{F}^{Z}(\infty)$-measurable since the process $A(\cdot)$ is the inverse of the $\mathbf{F}^{Z}$-adapted process $\Upsilon(\cdot)$. This means that the paths of $X(\cdot)$ are determined uniquely from those of $Z(\cdot)$.

Note furthermore, that we have

$$
A(t)=\int_{0}^{t} \frac{1}{\Upsilon^{\prime}(A(s))} \mathrm{d} s=\int_{0}^{t}[\mathcal{T}(N(A(s)))]^{2} \mathrm{~d} s=\int_{0}^{t}[\mathcal{T}(X(s))]^{2} \mathrm{~d} s
$$

\footnotetext{
${ }^{2}$ Note again that since the process $\Upsilon(\cdot)$ is strictly increasing, the inverse process $A(\cdot)$ is welldefined even if $\Upsilon(\cdot)$ does not satisfy the assumptions (P1) and (P2). These assumptions are equivalent to (2.44) and sufficient conditions are discussed in Section 2.3.
} 
Chapter 2. System of SDEs for the Generalized Volatility-Stabilized

in accordance with (2.43); this means that $A(\cdot)$ is adapted to the filtration $\mathbf{F}^{X}=$ $\left\{\mathcal{F}^{X}(t)\right\}_{0 \leq t<\infty}$, where

$$
\mathcal{F}^{X}(t) \triangleq \sigma\left(X_{i}(s): 0 \leq s \leq t, i=1, \ldots, n\right), \quad 0 \leq t<\infty .
$$

The processes $N_{i}(\cdot)$ of $(2.65)$ are themselves independent one-dimensional diffusions with state-space $I=(0, \infty)$ and dynamics

$$
\begin{aligned}
& \mathrm{d} N_{i}(\theta)=\frac{\alpha_{i}+\sigma^{2}}{2}\left(N_{i}(\theta)\right)^{1-2 \beta} \mathrm{d} \theta+\sigma\left(N_{i}(\theta)\right)^{1-\beta} \mathrm{d} V_{i}(\theta), \quad 0 \leq \theta<\infty \\
& N_{i}(0)=x_{i}>0, \quad i=1, \ldots, n
\end{aligned}
$$

as in (2.52). Hence, for the processes $X_{i}(\cdot)$ defined in (2.66) we obtain the following equations

$$
\begin{aligned}
X_{i}(t) & =N_{i}(A(t)) \\
& =x_{i}+\frac{\alpha_{i}+\sigma^{2}}{2} \int_{0}^{t}\left(N_{i}(A(s))\right)^{1-2 \beta} A^{\prime}(s) \mathrm{d} s+\sigma \int_{0}^{t}\left(N_{i}(\theta)\right)^{1-\beta} \mathrm{d} V_{i}(A(s))
\end{aligned}
$$

Consider now the continuous local martingales

$$
W_{i}(t) \triangleq \int_{0}^{t} \frac{\mathrm{d} V_{i}(A(s))}{\sqrt{A^{\prime}(s)}}=\int_{0}^{t} \frac{\mathrm{d} V_{i}(A(s))}{\mathcal{T}(N(A(s)))}=\int_{0}^{A(t)} \frac{\mathrm{d} V_{i}(\xi)}{\mathcal{T}(N(\xi))}, \quad 0 \leq t<\infty
$$

of the filtration $\mathbf{G}$ defined in (2.64), for $i=1, \ldots, n$. Their (cross-)variations are given as

$$
\left\langle W_{i}, W_{j}\right\rangle(t)=\delta_{i j} \int_{0}^{A(t)} \frac{1}{[\mathcal{T}(N(\xi))]^{2}} \mathrm{~d} \xi=\delta_{i j} \int_{0}^{A(t)} \Upsilon^{\prime}(\xi) \mathrm{d} \xi=\delta_{i j} t, \quad t \geq 0,
$$

thus $W_{1}(\cdot), \ldots, W_{n}(\cdot)$ are independent Brownian motions. Moreover, in terms of these processes and using the representation in (2.67), we can write the equations in $(2.68)$ as

$$
X_{i}(t)=x_{i}+\frac{\alpha_{i}+\sigma^{2}}{2} \int_{0}^{t} \frac{[\mathcal{T}(N(A(s)))]^{2}}{\left(N_{i}(A(s))\right)^{2 \beta-1}} \mathrm{~d} s+\sigma \int_{0}^{t} \frac{\mathcal{T}(N(A(s)))}{\left(N_{i}(A(s))\right)^{\beta-1}} \mathrm{~d} W_{i}(s)
$$


Chapter 2. System of SDEs for the Generalized Volatility-Stabilized

$$
=x_{i}+\frac{\alpha_{i}+\sigma^{2}}{2} \int_{0}^{t} \frac{[\mathcal{T}(X(s))]^{2}}{\left(X_{i}(s)\right)^{2 \beta-1}} \mathrm{~d} s+\sigma \int_{0}^{t} \frac{\mathcal{T}(X(s))}{\left(X_{i}(s)\right)^{\beta-1}} \mathrm{~d} W_{i}(s),
$$

which is precisely (2.4). Note also that each

$$
W_{i}(t)=\int_{0}^{t}\left[\frac{\left(X_{i}(s)\right)^{\beta-1}}{\sigma \mathcal{T}(X(s))} \mathrm{d} X_{i}(s)-\frac{\alpha_{i}+\sigma^{2}}{2} \frac{\mathcal{T}(X(s))}{\left(X_{i}(s)\right)^{\beta}} \mathrm{d} s\right]
$$

is $\mathcal{F}^{X}(t)$-measurable, so the independent Brownian motions $W_{1}(\cdot), \ldots, W_{n}(\cdot)$ are $\mathbf{F}^{X}$-adapted.

In other words, $(\Omega, \mathcal{F}, \mathbb{P}), \mathbf{F}^{X},(X(\cdot), W(\cdot))$ constitutes a weak solution of the system of equations (2.4), which is equivalent to the system in (2.1). According to our discussion in the Analysis section, uniqueness in distribution holds for this system and the constructed solution does not explode in finite time assuming that (2.44) is satisfied, i.e. the properties (P1) and (P2) stated in (2.62) and (2.63) are satisfied (we will discuss sufficient conditions for this in more detail in the following section).

Let us summarize the results of this section in the following proposition.

Proposition 2.1. Assume $K(\cdot):(0, \infty)^{n} \rightarrow(0, \infty)$ is a measurable function and that the properties (P1) and (P2) stated in (2.62) and (2.63) are satisfied. Then there exist a unique in distribution weak solution for the system of equations (2.1), and it does not explode in finite time.

Remark: We note that the properties (P1), (P2) of (2.62), (2.63) are not necessary conditions for the existence of a weak solution. As is shown in Pickova (2013), even if property $(\mathrm{P} 1)$ of $(2.62)$ is not satisfied there still exists a non-exploding weak solution (at least, on an extension of the probability space). However, if property (P2) of (2.63) is not satisfied, then this solution may explode in finite time. 
Remark: It is possible to extend the results of this section also to the case of pathdependent $K(\cdot)$; one would need to introduce slightly more complicated notation, but the whole construction would still hold and the solution will be unique in distribution and non-exploding, once the appropriate versions of conditions (P1) and (P2) are satisfied.

\subsection{Discussion of conditions on $K(\cdot)$}

In this section we will discuss conditions on the function $K(\cdot)$, under which the time-change process $\Upsilon(\cdot)$, defined in (2.61), satisfies the properties (P1) and (P2) stated in (2.62) and (2.63), respectively. The main tool in proving the statements below is finding bounds in terms of integral functionals of one-dimensional squaredBessel processes (respectively, functionals of one-dimensional Bessel processes), and applying results known for these functionals.

\subsubsection{Growth Conditions on $K(\cdot)$ to ensure property $(\mathbf{P} 1)$}

In the following proposition, we state sufficient growth conditions on the function $K(\cdot)$, that is, conditions implying that the time-change process $\Upsilon(\cdot)$ defined in $(2.61)$ satisfies property $(\mathrm{P} 1)$ in $(2.62)$, namely, $\Upsilon(\theta)<\infty$ a.s. for all $\theta \in(0, \infty)$ (i.e. it does not explode in finite time).

Let us consider a measurable function $K(\cdot):(0, \infty)^{n} \rightarrow(0, \infty)$ and the following conditions:

1. There exists an integer $\nu \in\{1, \ldots, n\}$ and a measurable function $f:(0, \infty) \rightarrow$ $(0, \infty)$ such that

$$
K(x) \geq f\left(x_{\nu}\right), \quad \forall x=\left(x_{1}, \ldots, x_{n}\right) \in(0, \infty)^{n}
$$


Chapter 2. System of SDEs for the Generalized Volatility-Stabilized Processes

and the function $u \mapsto\left[u f\left(u^{1 / \beta}\right)\right]^{-2}$ is locally integrable on $(0, \infty)$;

2. There exists an integer $k \in\{1, \ldots, n\}$, a set of $k$ integers $\nu_{1}, \ldots, \nu_{k} \in\{1, \ldots, n\}$, and measurable functions $f_{1}, \ldots, f_{k}:(0, \infty) \rightarrow(0, \infty)$, such that

$$
K(x) \geq f_{1}\left(x_{\nu_{1}}\right) \wedge \ldots \wedge f_{k}\left(x_{\nu_{k}}\right), \quad \forall x=\left(x_{1}, \ldots, x_{n}\right) \in(0, \infty)^{n}
$$

where the functions $u \mapsto\left[u f_{i}\left(u^{1 / \beta}\right)\right]^{-2}$ are locally integrable on $(0, \infty)$, for all $i \in\{1, \ldots, k\}$;

3. There exists a non-increasing measurable function $f:(0, \infty) \rightarrow(0, \infty)$ such that

$$
K(x) \geq f\left(\|x\|_{2 \beta}\right), \quad \forall x=\left(x_{1}, \ldots, x_{n}\right) \in(0, \infty)^{n},
$$

and the function $u \mapsto\left[u f\left(u^{1 / \beta}\right)\right]^{-2}$ is locally integrable on $(0, \infty)$, where we define $\|x\|_{p}:=\left(x_{1}^{p}+\cdots+x_{n}^{p}\right)^{1 / p}$ for any $p>0$;

4. There exist constants $C_{g}>0, k_{p} \geq 0$ and $K_{p} \geq 0$ such that

$$
K(x) \geq C_{g}\left[\left(\sum_{\nu=1}^{n} x_{\nu}\right)^{k_{p}} \wedge\left(\sum_{\nu=1}^{n} x_{\nu}\right)^{-K_{p}}\right], \quad \forall x \in(0, \infty)^{n}
$$

where we define $a \wedge b:=\min \{a, b\}$ for any $a, b \in \mathbb{R}$.

Proposition 2.2. Assume $K(\cdot):(0, \infty)^{n} \rightarrow(0, \infty)$ is a measurable function, and one of the above conditions (2.69), (2.70), (2.71) or (2.72) holds. Then the timechange process $\Upsilon(\cdot)$ defined in (2.61) satisfies property (P1) in (2.62).

Proof. Recalling the definition of the process $\Upsilon(\cdot)$ in $(2.61)$, with $\mathcal{T}(\cdot)$ defined in (2.3), we have

$$
\Upsilon(\theta)=\int_{0}^{\theta}\left[\mathcal{T}\left((\beta \sigma)^{\frac{1}{\beta}}\left(Z_{1}(\xi)\right)^{\frac{1}{2 \beta}}, \ldots,(\beta \sigma)^{\frac{1}{\beta}}\left(Z_{n}(\xi)\right)^{\frac{1}{2 \beta}}\right)\right]^{-2} \mathrm{~d} \xi
$$


Chapter 2. System of SDEs for the Generalized Volatility-Stabilized

$$
=C_{\beta, \sigma, n} \int_{0}^{\theta}\left[K\left(\left(Z_{1}(\xi)\right)^{\frac{1}{2 \beta}}, \ldots,\left(Z_{n}(\xi)\right)^{\frac{1}{2 \beta}}\right)\right]^{-2}\left[\sum_{j=1}^{n}\left(Z_{j}(\xi)\right)^{\frac{1}{2 \beta}}\right]^{-2 \beta} \mathrm{d} \xi
$$

where $C_{\beta, \sigma, n}$ is a scaling constant depending only on $\beta, \sigma$, and $n$.

In order to show that the process $\Upsilon(\cdot)$ satisfies property (P1) in (2.62), note that in the first case, assuming (2.69), we obtain

$$
\begin{aligned}
\Upsilon(\theta) / C_{\beta, \sigma, n} & \leq \int_{0}^{\theta}\left[f\left(\left(Z_{\nu}(\xi)\right)^{\frac{1}{2 \beta}}\right)\right]^{-2}\left(Z_{\nu}(\xi)\right)^{-1} \mathrm{~d} \xi \\
& =\int_{0}^{\theta}\left[R_{\nu}(\xi) f\left(\left(R_{\nu}(\xi)\right)^{\frac{1}{\beta}}\right)\right]^{-2} \mathrm{~d} \xi
\end{aligned}
$$

where we noted that $R_{\nu}(\cdot):=\sqrt{Z_{\nu}(\cdot)}$ is a Bessel process in dimension $m_{\nu} \geq 2$. The claim follows from Proposition A.1, and from the assumption that the function $\left[u f\left(u^{1 / \beta}\right)\right]^{-2}$ is locally integrable on $(0, \infty)$.

In the second case, assuming (2.70), we have

$$
\begin{aligned}
\Upsilon(\theta) / C_{\beta, \sigma, n} \leq \int_{0}^{\theta} & {\left[\min _{i=1, \ldots, k} f_{i}\left(\left(Z_{\nu_{i}}(\xi)\right)^{\frac{1}{2 \beta}}\right)\right]^{-2}\left[\sum_{j=1}^{n}\left(Z_{j}(\xi)\right)^{\frac{1}{2 \beta}}\right]^{-2 \beta} \mathrm{d} \xi } \\
\leq & \int_{0}^{\theta} \sum_{i=1}^{k}\left[f_{i}\left(\left(Z_{\nu_{i}}(\xi)\right)^{\frac{1}{2 \beta}}\right)\right]^{-2}\left[\sum_{j=1}^{n}\left(Z_{j}(\xi)\right)^{\frac{1}{2 \beta}}\right]^{-2 \beta} \mathrm{d} \xi \\
\leq & \sum_{i=1}^{k} \int_{0}^{\theta}\left[f_{i}\left(\left(Z_{\nu_{i}}(\xi)\right)^{\frac{1}{2 \beta}}\right)\right]^{-2}\left(Z_{\nu_{i}}(\xi)\right)^{-1} \mathrm{~d} \xi
\end{aligned}
$$

In other words, we have the following bound

$$
\Upsilon(\theta) / C_{\beta, \sigma, n} \leq \sum_{i=1}^{k} \int_{0}^{\theta}\left[R_{\nu_{i}}(\xi) f_{i}\left(\left(R_{\nu_{i}}(\xi)\right)^{\frac{1}{\beta}}\right)\right]^{-2} \mathrm{~d} \xi
$$

where we have denoted $R_{\nu_{i}}(\cdot):=\sqrt{Z_{\nu_{i}}(\cdot)}$ a Bessel process in dimension $m_{\nu_{i}} \geq 2$, for $i=1, \ldots, k$. The claim again follows from Proposition A.1, and from the assumption that functions $\left[u f_{i}\left(u^{1 / \beta}\right)\right]^{-2}$ are locally integrable on $(0, \infty)$, for $i=1, \ldots, k$.

In the third case, using (2.71) and noting that $f(\cdot)$ is assumed to be a nonincreasing function, we obtain

$$
\Upsilon(\theta) / C_{\beta, \sigma, n} \leq \int_{0}^{\theta}\left[f\left\|\left(\left(Z_{1}(\xi)\right)^{\frac{1}{2 \beta}}, \ldots,\left(Z_{n}(\xi)\right)^{\frac{1}{2 \beta}}\right)\right\|_{2 \beta}\right]^{-2}\left[\sum_{j=1}^{n}\left(Z_{j}(\xi)\right)^{\frac{1}{2 \beta}}\right]^{-2 \beta} \mathrm{d} \xi
$$


Chapter 2. System of SDEs for the Generalized Volatility-Stabilized

$$
\begin{aligned}
& \leq \int_{0}^{\theta}\left[f \|\left(Z_{1}(\xi), \ldots,\left(Z_{n}(\xi)\right) \|_{1}^{\frac{1}{2 \beta}}\right]^{-2}\left(Z_{1}(\xi)\right)^{-1} \mathrm{~d} \xi\right. \\
& \leq \int_{0}^{\theta}\left[f\left(\left(Z_{1}(\xi)\right)^{\frac{1}{2 \beta}}\right)\right]^{-2}\left(Z_{1}(\xi)\right)^{-1} \mathrm{~d} \xi
\end{aligned}
$$

In other words, if we define $R_{1}(\cdot):=\sqrt{Z_{1}(\cdot)}$, a Bessel process in dimension $m_{1} \geq 2$, we have the following bound

$$
\Upsilon(\theta) / C_{\beta, \sigma, n} \leq \int_{0}^{\theta}\left[R_{1}(\xi) f\left(\left(R_{1}(\xi)\right)^{\frac{1}{\beta}}\right)\right]^{-2} \mathrm{~d} \xi .
$$

The claim follows once again from Proposition A.1, and from the assumption that the function $\left[u f\left(u^{1 / \beta}\right)\right]^{-2}$ is locally integrable on $(0, \infty)$.

In the fourth case, combining (2.72) and (2.73) we have

$$
\begin{aligned}
& \Upsilon(\theta) / C_{\beta, \sigma, n} C_{g}^{-2} \\
& \leq \int_{0}^{\theta}\left[\left(\sum_{\nu=1}^{n}\left(Z_{\nu}(\xi)\right)^{\frac{1}{2 \beta}}\right)^{k_{p}} \wedge\left(\sum_{\nu=1}^{n}\left(Z_{\nu}(\xi)\right)^{\frac{1}{2 \beta}}\right)^{-K_{p}}\right]^{-2}\left[\sum_{j=1}^{n}\left(Z_{j}(\xi)\right)^{\frac{1}{2 \beta}}\right]^{-2 \beta} \mathrm{d} \xi \\
& \leq \int_{0}^{\theta}\left(\sum_{\nu=1}^{n}\left(Z_{\nu}(\xi)\right)^{\frac{1}{2 \beta}}\right)^{-2 k_{p}-2 \beta} \vee\left(\sum_{\nu=1}^{n}\left(Z_{\nu}(\xi)\right)^{\frac{1}{2 \beta}}\right)^{2 K_{p}-2 \beta} \mathrm{d} \xi
\end{aligned}
$$

Now, if $K_{p}<\beta$, we further have

$$
\begin{aligned}
\Upsilon(\theta) / C_{\beta, \sigma, n} & \leq C_{g}^{-2} \int_{0}^{\theta}\left(Z_{1}(\xi)\right)^{-1-\frac{k_{p}}{\beta}} \vee\left(Z_{1}(\xi)\right)^{-1+\frac{K_{p}}{\beta}} \mathrm{d} \xi \\
& \leq C_{g}^{-2} \int_{0}^{\theta}\left(R_{1}(\xi)\right)^{-2-2 \frac{k_{p}}{\beta}} \vee\left(R_{1}(\xi)\right)^{-2+2 \frac{K_{p}}{\beta}} \mathrm{d} \xi
\end{aligned}
$$

where we have defined $R_{1}(\cdot):=\sqrt{Z_{1}(\cdot)}$, a Bessel process in dimension $m_{1} \geq 2$. The claim follows once again from Proposition A.1, and from the fact that the function $\left[u^{-2-2 k_{p} / \beta} \vee u^{-2+2 K_{p} / \beta}\right]$ is locally integrable on $(0, \infty)$.

In case $K_{p} \geq \beta$, we obtain

$$
\begin{aligned}
\Upsilon(\theta) / C_{\beta, \sigma, n} & \leq C_{g}^{-2} \int_{0}^{\theta}\left(Z_{1}(\xi)\right)^{-1-\frac{k_{p}}{\beta}} \vee\left(n \max _{1 \leq j \leq n}\left\{\left(Z_{j}(\xi)\right)^{1 /(2 \beta)}\right\}\right)^{2 K_{p}-2 \beta} \mathrm{d} \xi \\
& \leq C_{g}^{-2} n^{2 K_{p}-2 \beta} \int_{0}^{\theta}\left(Z_{1}(\xi)\right)^{-1-\frac{k_{p}}{\beta}} \vee\left(\sum_{j=1}^{n} Z_{j}(\xi)\right)^{-1+K_{p} / \beta} \mathrm{d} \xi
\end{aligned}
$$


Furthermore, we have the following bound

$$
\begin{aligned}
\Upsilon(\theta) / C_{\beta, \sigma, n} & \leq C_{g}^{-2} n^{2 K_{p}-2 \beta}\left(\int_{0}^{\theta}\left(Z_{1}(\xi)\right)^{-1-\frac{k_{p}}{\beta}} \mathrm{d} \xi+\int_{0}^{\theta}(Z(\xi))^{-1+K_{p} / \beta} \mathrm{d} \xi\right) \\
& =C_{g}^{-2} n^{2 K_{p}-2 \beta}\left(\int_{0}^{\theta}\left(R_{1}(\xi)\right)^{-2-2 \frac{k_{p}}{\beta}} \mathrm{d} \xi+\int_{0}^{\theta}(R(\xi))^{-2+2 K_{p} / \beta} \mathrm{d} \xi\right)
\end{aligned}
$$

where we have noted that $R_{1}(\cdot):=\sqrt{Z_{1}(\cdot)}$ is a Bessel process in dimension $m_{1} \geq 2$, respectively $R(\cdot):=\sqrt{Z(\cdot)}=\sqrt{Z_{1}(\cdot)+\ldots+Z_{n}(\cdot)}$ is a Bessel process in dimension $m=m_{1}+\ldots+m_{n}>2$. The claim follows once again from Proposition A.1, and the fact that the function $\left[u^{-2-2 k_{p} / \beta}\right]$, as well as the function $\left[u^{-2+2 K_{p} / \beta}\right]$, is locally integrable on $(0, \infty)$.

\subsubsection{Conditions on $K(\cdot)$ to ensure property (P2)}

In the following proposition, we state conditions on $K(\cdot)$ that are sufficient so that the process $A(\cdot)$ does not explode in finite time. In other words, the stated conditions imply that the time-change process $\Upsilon(\cdot)$ defined in (2.61), with $\mathcal{T}(\cdot)$ defined in (2.3), satisfies property (P2) in (2.63), that is $\Upsilon(\theta) \rightarrow \infty$ a.s. as $\theta \rightarrow \infty$. As a consequence, explosion of the system in (2.1) does not occur in finite time.

Proposition 2.3. Assume $K(\cdot):(0, \infty)^{n} \rightarrow(0, \infty)$ is a measurable function and there exists a measurable function $f:(0, \infty) \rightarrow(0, \infty)$ such that for all $x=\left(x_{1}, \ldots, x_{n}\right) \in(0, \infty)^{n}$

$$
K(x) \leq f\left(\|x\|_{2 \beta}\right), \quad \text { and } \quad \int_{a}^{\infty}\left[u f^{2}\left(u^{1 / \beta}\right)\right]^{-1} \mathrm{~d} u=\infty,
$$

where $a:=\sum_{i=1}^{n} Z_{i}(0)=1 /(\beta \sigma)^{2} \sum_{i=1}^{n} x_{i}^{2 \beta}>0$, and $\|x\|_{p}:=\left(x_{1}^{p}+\cdots+x_{n}^{p}\right)^{1 / p}$ for any $p>0$. Then the time-change process $\Upsilon(\cdot)$ defined in (2.61) satisfies property (P2) in (2.63).

Proof. Recalling (2.73) and noticing the following inequalities

$$
\left[\sum_{j=1}^{n}\left(Z_{j}(\xi)\right)^{\frac{1}{2 \beta}}\right]^{-2 \beta} \geq\left(n \max _{1 \leq j \leq n}\left\{\left(Z_{j}(\xi)\right)^{\frac{1}{2 \beta}}\right\}\right)^{-2 \beta}
$$


Chapter 2. System of SDEs for the Generalized Volatility-Stabilized

$$
=n^{-2 \beta}\left(\max _{1 \leq j \leq n}\left\{Z_{j}(\xi)\right\}\right)^{-1} \geq n^{-2 \beta}\left(\sum_{j=1}^{n} Z_{j}(\xi)\right)^{-1}
$$

we obtain from $(2.74)$

$$
\begin{aligned}
\Upsilon(\theta) / C_{\beta, \sigma, n} & \geq \int_{0}^{\theta}\left[f\left(\left\|\left(\left(Z_{1}(\xi)\right)^{\frac{1}{2 \beta}}, \ldots,\left(Z_{n}(\xi)\right)^{\frac{1}{2 \beta}}\right)\right\|_{2 \beta}\right)\right]^{-2}\left[\sum_{j=1}^{n}\left(Z_{j}(\xi)\right)^{\frac{1}{2 \beta}}\right]^{-2 \beta} \mathrm{d} \xi \\
& \geq n^{-2 \beta} \int_{0}^{\theta}\left[f\left(\|\left(Z_{1}(\xi), \ldots,\left(Z_{n}(\xi)\right) \|_{1}^{\frac{1}{2 \beta}}\right)\right]^{-2}\left(\sum_{j=1}^{n} Z_{j}(\xi)\right)^{-1} \mathrm{~d} \xi\right.
\end{aligned}
$$

Therefore

$$
\Upsilon(\theta) / C_{\beta, \sigma, n} \geq n^{-2 \beta} \int_{0}^{\theta}\left[R(\xi) f\left((R(\xi))^{\frac{1}{\beta}}\right)\right]^{-2} \mathrm{~d} \xi
$$

where we have noted that $R(\cdot):=\sqrt{Z(\cdot)}=\sqrt{Z_{1}(\cdot)+\ldots+Z_{n}(\cdot)}$ is a Bessel process in dimension $m=m_{1}+\ldots+m_{n}>2$ starting from $R(0)=\sqrt{a}$. The claim follows from Proposition A.2, and from the assumption in (2.74).

Remark: It is easy to see that if $K(\cdot)$ is bounded, i.e. if there exist real constants $K_{\max }>K_{\min }>0$ such that $K_{\min } \leq K(x) \leq K_{\max }$ for all $x \in(0, \infty)^{n}$, then conditions (2.69), (2.70), (2.71), (2.72), and (2.74) are trivially satisfied. Therefore, if $K(\cdot)$ is bounded, the system of equations in (2.1) has a weak solution that is unique in the sense of the probability distribution and does not explode in finite time. Notice that $K(\cdot)$ need not be continuous.

\subsection{Pathwise Uniqueness and Strength}

After constructing a weak solution, a natural question arises: Is the constructed solution strong? In other words, one would like to know if the processes $X_{1}(\cdot), \ldots$, $X_{n}(\cdot)$ are adapted to the filtration $\mathbf{F}^{W}=\left\{\mathcal{F}^{W}(t)\right\}_{0 \leq t<\infty}$ of the driving Brownian motion $W(\cdot)$ in $(2.1)$, where we have denoted

$$
\mathcal{F}^{W}(t) \triangleq \sigma\left(W_{i}(s): 0 \leq s \leq t, i=1, \ldots, n\right), \quad 0 \leq t<\infty .
$$


In this section, we argue that under certain additional conditions, pathwise uniqueness holds for the system of equations $(2.1)$ in the state space $(0, \infty)^{n}$. As a consequence, we obtain strength thanks to the results of Yamada and Watanabe (1971).

We will use the following notation for the (Euclidean) $L_{2}$-norm $\|\cdot\|_{2}$, resp. the $L_{1}$-norm $\|\cdot\|_{1}$,

$$
\|u\|_{1} \triangleq \sum_{\nu=1}^{n}\left|u_{\nu}\right|, \quad\|u\|_{2} \triangleq\left(\sum_{\nu=1}^{n} u_{\nu}^{2}\right)^{1 / 2}, \quad u \in \mathbb{R}^{n} .
$$

Assume that $K(\cdot)$ is continuous and bounded from above, i.e., assume there exists a constant $K_{\max }>0$ such that

$$
K(x) \leq K_{\max }, \quad \forall x \in(0, \infty)^{n}
$$

Then the system of stochastic differential equations in (2.1) has a non-exploding weak solution which is unique in distribution (according to the results of the previous section), and is equivalent to the system

$$
\begin{array}{r}
\mathrm{d} X_{i}(t)=\frac{\alpha_{i}+\sigma^{2}}{2}\left[X_{i}(t)\right]^{1-2 \beta}\left(\sum_{\nu=1}^{n} X_{\nu}(t)\right)^{2 \beta}[K(X(t))]^{2} \mathrm{~d} t \\
+\sigma\left[X_{i}(t)\right]^{1-\beta}\left(\sum_{\nu=1}^{n} X_{\nu}(t)\right)^{\beta} K(X(t)) \mathrm{d} W_{i}(t),
\end{array}
$$

for $i=1, \ldots, n$, with the state process $X(\cdot)=\left(X_{1}(\cdot), \ldots, X_{n}(\cdot)\right)$ taking values in the strictly positive orthant $(0, \infty)^{n}$. If we define

$$
Y_{i}(t) \triangleq \log X_{i}(t), 0 \leq t<\infty, \quad i=1, \ldots, n,
$$

we can rewrite the system of stochastic differential equations in (2.78) as

$$
\begin{aligned}
\mathrm{d} Y_{i}(t)=\frac{\alpha_{i}}{2} \mathrm{e}^{-2 \beta Y_{i}(t)}\left(\sum_{\nu=1}^{n} \mathrm{e}^{Y_{\nu}(t)}\right)^{2 \beta}[K(\xi(Y(t)))]^{2} \mathrm{~d} t \\
\quad+\sigma \mathrm{e}^{-\beta Y_{i}(t)}\left(\sum_{\nu=1}^{n} \mathrm{e}^{Y_{\nu}(t)}\right)^{\beta} K(\xi(Y(t))) \mathrm{d} W_{i}(t),
\end{aligned}
$$


for $i=1, \ldots, n$, where the state process $Y(\cdot)=\left(Y_{1}(\cdot), \ldots, Y_{n}(\cdot)\right)$ takes values in $\mathbb{R}^{n}$, and we have defined the $C^{\infty}$-function $\xi(\cdot): \mathbb{R}^{n} \mapsto(0, \infty)^{n}$ as

$$
\xi(y):=\left(\mathrm{e}^{y_{1}}, \ldots, \mathrm{e}^{y_{n}}\right), \forall y \in \mathbb{R}^{n} .
$$

In addition to the assumption that $K(\cdot)$ is bounded, assume that $K(\cdot)$ is differentiable in the strictly positive orthant $(0, \infty)^{n}$, and all of its partial derivatives are locally bounded. Then, for any positive integer $k$ there exists a constant $D_{k}$ such that

$$
\|\nabla K(\xi(y))\|_{1} \leq D_{k}, \forall y \in B_{k}
$$

where we denoted

$$
B_{k}:=\left\{u=\left(u_{1}, \ldots, u_{n}\right) \in \mathbb{R}^{n} \mid\|u\|_{1} \leq k\right\}, k \geq 1
$$

We claim that under assumptions (2.77) and (2.81), namely if the function $K(\cdot)$ is bounded and has locally bounded partial derivatives, pathwise uniqueness holds for the system of equations (2.80) in the state space $\mathbb{R}^{n}$, thus also for $(2.78)$ in the strictly positive orthant $(0, \infty)^{n}$ thanks to the definition in $(2.79)$.

We shall show that the coefficients of the equations in (2.80) are locally Lipschitz in the state space. First, fix an arbitrary $j \in\{1, \ldots, n\}$ and $p>0$, and consider a function $g_{j}^{p}(\cdot): \mathbb{R}^{n} \mapsto(0, \infty)$ defined as follows

$$
g_{j}^{p}(y) \triangleq \mathrm{e}^{-p y_{j}}\left(\sum_{\nu=1}^{n} \mathrm{e}^{y_{\nu}}\right)^{p}, \quad y \in \mathbb{R}^{n} .
$$

It is easy to see that all partial derivatives of the function $g_{j}^{p}(\cdot)$ are bounded on compact sets in $\mathbb{R}^{n}$. Therefore, for any positive integer $k$ and $u, v \in B_{k}$, where $B_{k}$ is defined as in (2.82), there exist a constant $C_{p, k}$ (which depends only on $k$ and $p$ ) such that

$$
\left|g_{j}^{p}(u)-g_{j}^{p}(v)\right| \leq C_{p, k}\|u-v\|_{1}, \quad \forall u, v \in B_{k}
$$


The constant $C_{p, k}$ can be chosen as $C_{p, k}:=p \mathrm{e}^{p k}\left[n^{|p-1|} \mathrm{e}^{k(|p-1|+1)}+(n k)^{p}\right]$.

The corresponding drift vector $b(\cdot)=\left\{b_{i}(\cdot)\right\}_{1 \leq i \leq n}$ and the dispersion matrix $s(\cdot)=\left\{s_{i j}(\cdot)\right\}_{1 \leq i, j \leq n}$ in $(2.80)$ are given by

$$
b_{i}(y)=\frac{\alpha_{i}}{2} g_{i}^{2 \beta}(y)[K(\xi(y))]^{2}, \quad s_{i j}(y)=\sigma g_{i}^{\beta}(y) K(\xi(y)) \delta_{i j},
$$

respectively, for $y \in \mathbb{R}^{n}, 1 \leq i, j \leq n$, recalling the definition in (2.83). Thanks to the bounds in (2.81) and (2.84), and since for any positive integer $k$ and $p>0$ we have $\left|g_{j}^{p}(y)\right| \leq \mathrm{e}^{p k}\left(n \mathrm{e}^{k}\right)^{p}$ for $y \in B_{k}$, all the partial derivatives of the functions in (2.85) are locally bounded, in particular for any $1 \leq i, j \leq n$, any positive integer $k$ and any $y \in B_{k}$, we have

$$
\begin{aligned}
\left|\frac{\partial}{\partial y_{j}} b_{i}(y)\right| & \leq \frac{\alpha_{i}}{2}\left[C_{2 \beta, k} \cdot K_{\max }^{2}+\mathrm{e}^{2 \beta}\left(n \mathrm{e}^{k}\right)^{2 \beta} \cdot 2 K_{\max } \cdot D_{k} \cdot \mathrm{e}^{k}\right] \\
\left|\frac{\partial}{\partial y_{j}} s_{i i}(y)\right| & \leq \sigma\left[C_{\beta, k} \cdot K_{\max }+\mathrm{e}^{\beta}\left(n \mathrm{e}^{k}\right)^{\beta} \cdot D_{k} \cdot \mathrm{e}^{k}\right] .
\end{aligned}
$$

Therefore, there exists a constant $\widetilde{K}_{k, \alpha, \beta, \sigma, n}$ which depends only on the values of $k, \alpha_{\max }, \beta, \sigma$ and $n$, such that for any positive integer $k$ and any $u, v \in B_{k}$

$$
\|b(u)-b(v)\|_{2}+\|s(u)-s(v)\|_{2} \leq \widetilde{K}_{k, \alpha, \beta, \sigma, n}\|u-v\|_{2}
$$

In other words, the coefficients in (2.80) are locally Lipschitz in the state space $\mathbb{R}^{n}$. Hence, pathwise uniqueness holds for $(2.80)$, thanks to the Itô theory (see for instance Theorem 5.2.5 in Karatzas and Shreve (1991)), which, in conjunction with the existence of a weak solution, implies strength (thanks to the results of Yamada and Watanabe (1971)). In conclusion, the system in (2.1) admits a pathwise unique strong solution under the above stated assumptions on $K(\cdot)$. Let us summarize this result in the following proposition.

Proposition 2.4. Assume $K(\cdot):(0, \infty)^{n} \rightarrow(0, \infty)$ is a continuous and differentiable function such that (2.77) and (2.81) are satisfied (namely, it is bounded and has locally bounded partial derivatives). Then the system in (2.1) admits a pathwise unique strong solution. 
Chapter 2. System of SDEs for the Generalized Volatility-Stabilized Processes

Remark: The assumptions on $K(\cdot)$ can be further relaxed. In particular, if $K(\cdot)$ is bounded and locally Lipschitz, then again the coefficients in (2.80) are locally Lipschitz in the state space $\mathbb{R}^{n}$, and pathwise uniqueness holds for (2.80), respectively $(2.1)$. 


\section{Chapter 3}

\section{Generalized Volatility-Stabilized processes in Stochastic Portfolio Theory}

In this chapter we will describe the properties and the role of the Generalized Volatility-Stabilized processes within the framework of Stochastic Portfolio Theory. Let us start with a brief overview of the basic concepts of stochastic portfolio theory, introduce some definitions and statements that we will use in the following sections. For more details we refer the reader to the monograph Fernholz (2002) and to the survey paper Fernholz and Karatzas (2009), as well as the references mentioned there. 
Chapter 3. Generalized Volatility-Stabilized processes in Stochastic Portfolio Theory

\subsection{Basic Concepts of Stochastic Portfolio Theory}

Consider a model $\mathcal{M}$ for a financial market consisting of $n$ stocks with capitalizations $X_{1}(\cdot) \ldots, X_{n}(\cdot)$

$$
\begin{aligned}
\mathrm{d} X_{i}(t) & =X_{i}(t)\left(b_{i}(t) \mathrm{d} t+\sum_{\nu=1}^{d} \sigma_{i \nu}(t) \mathrm{d} W_{\nu}(t)\right), \\
X_{i}(0) & =x_{i}>0, \quad i=1, \ldots, n,
\end{aligned}
$$

driven by the $d$-dimensional Brownian motion $W(\cdot)=\left(W_{1}(\cdot), \ldots, W_{d}(\cdot)\right)$, with $d \geq n \geq 2$, on a complete probability space $(\Omega, \mathcal{F}, \mathbb{P})$ equipped with a filtration $\mathbf{F}=\{\mathcal{F}(t)\}_{0 \leq t<\infty}$. We shall assume that the vector-valued process $X(\cdot)=$ $\left(X_{1}(\cdot), \ldots, X_{n}(\cdot)\right)^{\prime}$ of stocks' capitalizations, as well as the vector-valued process $b(\cdot)=\left(b_{1}(\cdot), \ldots, b_{n}(\cdot)\right)^{\prime}$ of rates of return, and the $(n \times d)$-matrix-valued process $s(\cdot)=\left(s_{i \nu}\right)_{1 \leq i \leq n, 1 \leq \nu \leq d}$ of stock-price volatilities are all $\mathbf{F}$-progressively measurable, where the filtration $\mathbf{F}$ (which represents the "flow of information" in the market), is part of a weak solution to the system of stochastic differential equations in (3.1) and satisfies the usual conditions of right-continuity and augmentation by $\mathbb{P}$-negligible sets. Note, that it does not necessarily have to be the filtration generated by the Brownian motion itself.

We also assume that there exist a weak solution to the system of stochastic differential equations in (3.1). An example of a sufficient condition for this is that for every $T \in(0, \infty)$,

$$
\int_{0}^{T} \sum_{i=1}^{n}\left(\left|b_{i}(t)\right|+\sum_{\nu=1}^{d}\left(\sigma_{i \nu}(t)\right)^{2}\right) \mathrm{d} t<\infty, \quad \text { a.s. }
$$

Elementary stochastic calculus allows us to rewrite the system in (3.1) in the equivalent form

$$
\begin{aligned}
\mathrm{d}\left(\log X_{i}(t)\right) & =\gamma_{i}(t) \mathrm{d} t+\sum_{\nu=1}^{d} s_{i \nu}(t) \mathrm{d} W_{\nu}(t), \\
X_{i}(0) & =x_{i}>0, \quad i=1, \ldots, n,
\end{aligned}
$$


where we have introduced

$$
\gamma_{i}(t):=b_{i}(t)-\frac{1}{2} a_{i i}(t), \quad a_{i j}(t):=\sum_{\nu=1}^{d} s_{i \nu}(t) s_{j \nu}(t)=\left(s(t) s^{\prime}(t)\right)_{i j} .
$$

Here $a(\cdot)=\left(a_{i j}(\cdot)\right)_{1 \leq i, j \leq n}$ is the nonnegative definite matrix-valued covariance process of the stocks in the market, and $\gamma_{i}(\cdot)$ will further be referred to as the growth rate of the $i$ th stock.

Next, we define a long-only portfolio rule $\pi(\cdot)=\left(\pi_{1}(\cdot), \ldots, \pi_{n}(\cdot)\right)$, that is, an F-progressively measurable process, with values in the simplex

$$
\Delta^{n}=\left\{\left(x_{1}, \ldots, x_{n}\right) \in \mathbb{R}^{n} \mid x_{1} \geq 0, \ldots, x_{n} \geq 0 \text { and } x_{1}+\cdots+x_{n}=1\right\} .
$$

The quantity $\pi_{i}(t)$ is interpreted as the proportion of wealth invested in the $i$ th stock at time $t$.

The wealth process $V^{\omega, \pi}(t)$, which corresponds to a portfolio rule $\pi(\cdot)$ and some initial capital $\omega>0$, satisfies the stochastic differential equation

$$
\begin{aligned}
\frac{\mathrm{d} V^{\omega, \pi}(t)}{V^{\omega, \pi}(t)} & =\sum_{i}^{n} \pi_{i}(t) \frac{\mathrm{d} X_{i}(t)}{X_{i}(t)}=b_{\pi}(t) \mathrm{d} t+\sum_{\nu=1}^{d} s_{\pi \nu}(t) \mathrm{d} W_{\nu}(t), \\
V^{\omega, \pi}(0) & =\omega,
\end{aligned}
$$

where for $\nu=1, \ldots, d$ we defined

$$
b_{\pi}(t):=\sum_{i}^{n} \pi_{i}(t) b_{i}(t), \quad s_{\pi \nu}(t):=\sum_{i}^{n} \pi_{i}(t) s_{i \nu}(t),
$$

the rate of return and the volatility coefficients, respectively, associated with the portfolio $\pi(\cdot)$.

Using elementary stochastic calculus as in (3.3), we can write the dynamics for the wealth process in the equivalent form

$$
\mathrm{d}\left(\log V^{\omega, \pi}(t)\right)=\gamma_{\pi}(t) \mathrm{d} t+\sum_{\nu=1}^{d} s_{\pi \nu}(t) \mathrm{d} W_{\nu}(t), \quad V^{\omega, \pi}(0)=\omega
$$


Chapter 3. Generalized Volatility-Stabilized processes in Stochastic Portfolio Theory

where

$$
\gamma_{\pi}(t):=\sum_{i=1}^{n} \pi_{i}(t) \gamma_{i}(t)+\gamma_{\pi}^{*}(t)
$$

is the growth rate of the portfolio rule $\pi(\cdot)$, and

$$
\gamma_{\pi}^{*}(t)=\frac{1}{2}\left(\sum_{i=1}^{n} \pi_{i}(t) a_{i i}(t)-\sum_{i=1}^{n} \sum_{j=1}^{n} \pi_{i}(t) a_{i j} \pi_{j}(t)\right)
$$

is the excess growth rate of the portfolio $\pi(\cdot)$. The excess growth rate is always nonnegative for any long-only portfolio (see Lemma 3.3 in Fernholz and Karatzas (2009), and the alternative expression (3.10) below). Under certain conditions on the market (see Remark 3.2 in Fernholz and Karatzas (2009)), the excess growth rate is strictly positive for portfolios that do not concentrate their holdings in just one stock (that is if $\pi_{i}(t)>0$ holds a.s. for all $i=1, \ldots, n$ and $t \geq 0$ ).

Alternatively, the excess growth rate (3.9) can be written as

$$
\gamma_{\pi}^{*}(t)=\frac{1}{2} \sum_{i=1}^{n} \pi_{i}(t) \tau_{i i}^{\pi}(t)
$$

where we have denoted by $\tau_{i j}^{\pi}(\cdot)$ the individual stocks' covariance rates relative to the portfolio $\pi(\cdot)$,

$$
\tau_{i j}^{\pi}(t):=\sum_{k=1}^{n}\left(s_{i k}(t)-s_{\pi k}(t)\right)\left(s_{j k}(t)-s_{\pi k}(t)\right), \quad 1 \leq i, j \leq n .
$$

It is of key interest in mathematical finance, whether it is possible to outperform a given strategy. The assumption that such outperformance is not possible is common in classical mathematical finance, and one is usually interested in finding what conditions on the underlying model would prevent such "arbitrage". Stochastic portfolio theory, on the contrary, does not rule out arbitrage, and studies the market characteristics that allow for the possibility of outperformance. We say that a portfolio rule $\pi(\cdot)$ is an arbitrage opportunity relative to (equivalently, outperforms) the portfolio rule $\rho(\cdot)$ over the time horizon $[0, T]$, with $T>0$ a given real 
Chapter 3. Generalized Volatility-Stabilized processes in Stochastic Portfolio Theory

number, if

$$
\mathbb{P}\left[V^{\omega, \pi}(T) \geq V^{\omega, \rho}(T)\right]=1 \quad \text { and } \quad \mathbb{P}\left[V^{\omega, \pi}(T)>V^{\omega, \rho}(T)\right]>0 .
$$

Moreover, if we have

$$
\mathbb{P}\left[V^{\omega, \pi}(T)>V^{\omega, \rho}(T)\right]=1,
$$

we say that $\pi(\cdot)$ is a strong arbitrage opportunity relative to $\rho(\cdot)$ (equivalently, strongly outperforms $\rho(\cdot))$. The notion of relative arbitrage was introduced by Fernholz (2002). Under certain conditions on the market model $\mathcal{M}$, Fernholz and Karatzas (2009) show that the existence of relative arbitrage implies the absence of equivalent martingale measure in the market model $\mathcal{M}$. In the following, we shall use the notation $V^{\pi}(t):=V^{1, \pi}(t)$ whenever we start with initial capital $\omega=1$.

An important long-only portfolio (and also a natural choice for a reference portfolio) is the market portfolio, which invests in all stocks in proportion to their relative weights

$$
\mu_{i}(t):=\frac{X_{i}(t)}{X_{1}(t)+\ldots+X_{n}(t)}, \quad i=1, \ldots n .
$$

It is obvious from (3.6) that

$$
\frac{\mathrm{d} V^{\omega, \mu}(t)}{V^{\omega, \mu}(t)}=\frac{\mathrm{d}\left(X_{1}(t)+\ldots+X_{n}(t)\right)}{X_{1}(t)+\ldots+X_{n}(t)},
$$

and hence

$$
V^{\omega, \mu}(t)=\frac{\omega}{x}\left(X_{1}(t)+\ldots+X_{n}(t)\right), \quad 0 \leq t<\infty
$$

where $x=X_{1}(0)+\ldots+X_{n}(0)$. Therefore, holding the market portfolio amounts to owning the entire market in proportion to the initial capital.

We say, that the market model $\mathcal{M}$ of (3.1) and (3.2) is coherent if the relative capitalizations of (3.14) satisfy

$$
\lim _{T \rightarrow \infty} \frac{1}{T} \log \mu_{i}(T)=0, \quad \text { a.s., for each } i=1, \ldots, n,
$$


that is, if none of the stocks decline too rapidly with respect to the market as a whole.

The excess growth rate

$$
\gamma_{\mu}^{*}(\cdot)=\frac{1}{2} \sum_{i=1}^{n} \mu_{i}(\cdot) \tau_{i i}^{\mu}(\cdot)
$$

of the market portfolio measures the average relative variance rate of stocks in the market at any given time, as it is the average of relative market capitalization of the individual stocks' relative variance rates $\tau_{i i}^{\mu}(\cdot)$ with respect to the market. If it is bounded away from zero over a period of time, i.e., if there exists a constant $\zeta \in(0, \infty)$ such that

$$
\gamma_{\mu}^{*}(t) \geq \zeta, \quad \forall 0 \leq t \leq T
$$

holds with probability one, then certain types of portfolios outperform the market portfolio over the fixed time horizon $[0, T]$, with $T \in(0, \infty)$ a given real number, as was shown in Proposition 3.1 in Fernholz and Karatzas (2005). Another way to construct arbitrage opportunities is using the functionally generated portfolios (see Chapter III in Fernholz and Karatzas (2009)). In the next subsection, we will provide examples of arbitrage opportunities in a particular financial market, the Generalized Volatility-stabilized market (GVSM) which assumes the dynamics in (2.1) for stocks' capitalizations.

\subsection{Arbitrage Opportunities in Generalized Volatility-Stabilized Markets}

As we have already mentioned, the special case of the system in (2.1) with $\beta=1 / 2$ and $K(\cdot) \equiv 1$ corresponds to the volatility-stabilized market models which were introduced in Fernholz and Karatzas (2005). These markets exhibit one of the features observed in the real-life equity markets, in particular, the fact that small 
Chapter 3. Generalized Volatility-Stabilized processes in Stochastic Portfolio Theory

stocks tend to have bigger growth rates and are more volatile than the largest stocks in the markets. Fernholz and Karatzas (2005) discuss arbitrage opportunities that are present in these markets which we will now extend to the more general system in $(2.1)$.

Let us first consider the case of the system in $(2.1)$ with $K(\cdot) \equiv 1$ but $\beta>0$, not necessarily $1 / 2$, that is the following system of stochastic differential equations

$$
\mathrm{d}\left(\log X_{i}(t)\right)=\frac{\alpha_{i}}{2\left(\mu_{i}(t)\right)^{2 \beta}} \mathrm{d} t+\frac{\sigma}{\left(\mu_{i}(t)\right)^{\beta}} \mathrm{d} W_{i}(t), \quad i=1, \ldots, n
$$

or equivalently

$$
\mathrm{d} X_{i}(t)=\frac{\alpha_{i}+\sigma^{2}}{2}\left[X_{i}(t)\right]^{1-2 \beta}[S(t)]^{2 \beta} \mathrm{d} t+\sigma\left[X_{i}(t)\right]^{1-\beta}[S(t)]^{\beta} \mathrm{d} W_{i}(t)
$$

for all $i=1, \ldots, n$, where $\alpha_{i} \geq 0, \sigma>0, \beta>0$ are given constants, $\mu(\cdot)=$ $\left(\mu_{1}(\cdot), \ldots, \mu_{n}(\cdot)\right)$ is the vector of market weights

$$
\mu_{i}(t)=\frac{X_{i}(t)}{S(t)}=\frac{X_{i}(t)}{X_{1}(t)+\cdots+X_{n}(t)}, \quad i=1, \ldots, n
$$

and $\left(W_{1}(\cdot), \ldots, W_{n}(\cdot)\right)$ is $n$-dimensional Brownian motion.

\subsubsection{Excess growth rate of the market portfolio and the diversity weighted portfolio}

Assuming that the dynamics of the processes $X_{i}(\cdot)$ are described by the system of equations (3.16), the corresponding growth rates and volatilities are given by

$$
\gamma_{i}(t)=\frac{\alpha_{i}}{2}\left(\mu_{i}(t)\right)^{-2 \beta}, \quad s_{i \nu}(t)=\sigma\left(\mu_{i}(t)\right)^{-\beta} \delta_{i \nu}
$$

respectively. The covariance matrix is given by

$$
a_{i j}(t)=\left(s(t) s^{T}(t)\right)_{i j}=\sigma^{2}\left(\mu_{i}(t)\right)^{-2 \beta} \delta_{i j} .
$$

Therefore, we have for this model

$$
a_{\mu \mu}(t):=\mu(t) a(t) \mu^{T}(t)=\sigma^{2} \sum_{\nu=1}^{n}\left(\mu_{\nu}(t)\right)^{2-2 \beta}
$$


as well as

$$
\gamma_{\mu}^{*}(t)=\frac{1}{2}\left(\sum_{i=1}^{n} \mu_{i}(t) a_{i i}(t)-a_{\mu \mu}(t)\right)=\frac{\sigma^{2}}{2}\left(\sum_{i=1}^{n}\left(\mu_{i}(t)\right)^{1-2 \beta}-\sum_{i=1}^{n}\left(\mu_{i}(t)\right)^{2-2 \beta}\right) .
$$

Hence, the excess growth rate of the market portfolio in the model (3.16) is given by

$$
\gamma_{\mu}^{*}(t)=\frac{\sigma^{2}}{2} \sum_{i=1}^{n}\left(\mu_{i}(t)\right)^{1-2 \beta}\left(1-\mu_{i}(t)\right)
$$

Let us show that the excess growth rate $\gamma_{\mu}^{*}(t)$ of the market portfolio is bounded away from zero, if $1 / 2 \leq \beta<\infty$; indeed, since all market weights are smaller than 1 , we have then

$$
\gamma_{\mu}^{*}(t) \geq \frac{\sigma^{2}}{2} \sum_{i=1}^{n} 1\left(1-\mu_{i}(t)\right)=\frac{\sigma^{2}}{2}(n-1)>0
$$

for $n \geq 2$. Therefore, in this case, the condition (3.2) in Proposition 3.1 in Fernholz and Karatzas (2005) is satisfied with $\Gamma(t)=t \sigma^{2}(n-1) / 2$, and the model of $(3.16)$ admits relative arbitrage opportunities, namely there exist a sufficiently large real number $c>0$ such that the portfolio rule

$$
\pi_{i}(t):=\frac{c \mu_{i}(t)-\mu_{i}(t) \log \mu_{i}(t)}{c-\sum_{j=1}^{n} \mu_{j}(t) \log \mu_{j}(t)}, \quad j=1, \ldots n
$$

outperforms the market portfolio at least on the time-horizons $[0, T]$ with $T>$ $2 \log (n) /\left[\sigma^{2}(n-1)\right]$ (for the proof we refer the reader to Proposition 3.1 in Fernholz and Karatzas (2005)).

If $\beta \in(0,1 / 2)$, then $\gamma_{\mu}^{*}(t)$ can get arbitrarily close to zero whenever $\mu_{(1)}=$ $\max _{i=1, \ldots, n}\left\{\mu_{i}\right\}$ approaches one. Hence, condition (3.2) in Fernholz and Karatzas (2005) is not satisfied in this case.

However, one can construct a simple example of an arbitrage relative to the market portfolio that works for any value of $\beta>0$, as follows. With $0<p<1$ and $p \leq 2 \beta$, let us consider the so-called diversity-weighted portfolio

$$
\mu_{i}^{(p)}(t):=\frac{\left(\mu_{i}(t)\right)^{p}}{\sum_{j=1}^{n}\left(\mu_{j}(t)\right)^{p}}, \quad i=1, \ldots n .
$$


Chapter 3. Generalized Volatility-Stabilized processes in Stochastic Portfolio Theory

In model (3.16) we have $a_{i j}(t)=\sigma^{2}\left(\mu_{i}(t)\right)^{-2 \beta} \delta_{i j}$ for the elements of the variance/covariance matrix as in (3.17), so the excess growth rate of the portfolio $\mu^{(p)}(\cdot)$ is given by

$$
\begin{aligned}
2 \gamma_{\mu^{(p)}}^{*}(t) & =\sum_{i=1}^{n} \mu_{i}^{(p)}(t)\left(1-\mu_{i}^{(p)}(t)\right) a_{i i}(t) \\
& =\sum_{i=1}^{n} \frac{\left(\mu_{i}(t)\right)^{p}}{\sum_{j=1}^{n}\left(\mu_{j}(t)\right)^{p}}\left(1-\mu_{i}^{(p)}(t)\right) \sigma^{2}\left(\mu_{i}(t)\right)^{-2 \beta} \\
& \geq \sigma^{2} \sum_{i=1}^{n} \frac{\left(\mu_{i}(t)\right)^{p}}{\sum_{j=1}^{n}\left(\mu_{j}(t)\right)^{p}}\left(1-\mu_{i}^{(p)}(t)\right)\left(\mu_{i}(t)\right)^{-p} \\
& =\sigma^{2} \frac{\sum_{i=1}^{n}\left(1-\mu_{i}^{(p)}(t)\right)}{\sum_{j=1}^{n}\left(\mu_{j}(t)\right)^{p}}=\sigma^{2} \frac{n-1}{\sum_{i=1}^{n}\left(\mu_{i}(t)\right)^{p}}
\end{aligned}
$$

where the inequality is only valid if $p \leq 2 \beta$. Since the function $\Delta^{n} \ni \pi \mapsto \sum_{i=1}^{n}\left(\pi_{i}\right)^{p}$ attains its maximum, namely $n^{1-p}$, over the simplex $\Delta^{n}$ defined in (3.5), at the point $(1 / n, \ldots, 1 / n)$, we further have $\sum_{i=1}^{n}\left(\mu_{i}(t)\right)^{p} \leq n^{1-p}$, and therefore

$$
\gamma_{\mu(p)}^{*}(t) \geq \frac{\sigma^{2}}{2} \frac{n-1}{\sum_{i=1}^{n}\left(\mu_{i}(t)\right)^{p}} \geq \frac{\sigma^{2}}{2} \frac{n-1}{n^{1-p}} .
$$

If we introduce $\mathfrak{D}(\pi):=\left(\sum_{i=1}^{n} \pi_{i}^{p}\right)^{\frac{1}{p}}, \pi \in \Delta^{n}$, we can derive the following expression

$$
\log \left(\frac{V^{\mu^{(p)}(T)}}{V^{\mu}(T)}\right)=\log \left(\frac{\mathfrak{D}(\mu(T))}{\mathfrak{D}(\mu(0))}\right)+(1-p) \int_{0}^{T} \gamma_{\mu^{(p)}}^{*}(t) \mathrm{d} t, \quad \text { a.s. }
$$

for the wealth process $V^{\mu^{(p)}}(\cdot)$ of the diversity-weighted portfolio $\mu_{i}^{(p)}(\cdot)$ in (3.19) (see (7.5) in Fernholz and Karatzas (2009)). Notice that there is no stochastic integral term on the right hand side of the expression (3.21); this will allow us to make pathwise comparisons as follows: Since the function $\mathfrak{D}(\pi)$ takes values in $\left[1, n^{(1-p) / p}\right]$ for all $\pi \in \Delta^{n}$, and thanks to the lower bound on $\gamma_{\mu^{(p)}}^{*}(t)$ in (3.20) we obtain

$$
\log \left(\frac{V^{\mu^{(p)}}(T)}{V^{\mu}(T)}\right)=\log \left(\frac{\mathfrak{D}(\mu(T))}{\mathfrak{D}(\mu(0))}\right)+(1-p) \int_{0}^{T} \gamma_{\mu^{(p)}}^{*}(t) \mathrm{d} t
$$


Chapter 3. Generalized Volatility-Stabilized processes in Stochastic Portfolio Theory

$$
\geq(1-p)\left[\frac{(n-1) T \sigma^{2}}{2 n^{1-p}}-\frac{\log (n)}{p}\right]>0, \quad \text { a.s. }
$$

provided that

$$
T>\frac{2}{p \sigma^{2}} \cdot \frac{\log (n)}{n^{p}(1-1 / n)}=: T^{*}(\beta, \sigma, n) .
$$

In other words, the diversity-weighted portfolio of (3.19) outperforms the market portfolio over sufficiently large time horizons $[0, T]$, namely with $T>T^{*}(\beta, \sigma, n)$. Notice, that the threshold $T^{*}(\beta, \sigma, n)$ depends on the choice of parameter $\beta$ through the requirement $p \leq 2 \beta$. If the parameter $\beta \rightarrow 0$, we also need to choose $p \leq 2 \beta \rightarrow 0$, and then the threshold $T^{*}(\beta, \sigma, n) \rightarrow \infty$ (which means one needs to wait longer for the arbitrage). On the other hand, if either the volatility parameter $\sigma$ or the number of stocks $n$ increases to infinity, then $T^{*}(\beta, \sigma, n) \rightarrow 0$.

Remark: When $0<\beta<1 / 2$, we can show that if we choose $p=2 \beta$, then in the model of (3.16) the excess growth rate of the diversity-weighted portfolio dominates the excess growth rate of the market portfolio at any time $t>0$ :

$$
\gamma_{\mu(p)}^{*}(t) \geq \gamma_{\mu}^{*}(t)
$$

Indeed, we claim that the lower bound in the inequality

$$
\frac{2}{\sigma^{2}} \gamma_{\mu^{(p)}}^{*}(t) \geq \frac{n-1}{\sum_{i=1}^{n}\left(\mu_{i}(t)\right)^{p}}
$$

that we observed already in (3.20), dominates

$$
\frac{2}{\sigma^{2}} \gamma_{\mu}^{*}(t)=\sum_{i=1}^{n} \mu_{i}(t)\left(1-\mu_{i}(t)\right)\left(\mu_{i}(t)\right)^{-2 \beta}=\sum_{i=1}^{n}\left(1-\mu_{i}(t)\right)\left(\mu_{i}(t)\right)^{1-2 \beta} .
$$

For this, we have to check

$$
\begin{aligned}
\frac{2}{\sigma^{2}} \gamma_{\mu}^{*}(t) \cdot \sum_{i=1}^{n}\left(\mu_{i}(t)\right)^{p} & =\sum_{i=1}^{n}\left(\mu_{i}(t)\right)^{p} \sum_{i=1}^{n}\left(\mu_{i}(t)\right)^{1-2 \beta}-\sum_{i=1}^{n}\left(\mu_{i}(t)\right)^{p} \sum_{i=1}^{n}\left(\mu_{i}(t)\right)^{2(1-\beta)} \\
& \leq n-1 .
\end{aligned}
$$


Using the Cauchy-Schwarz inequality, we show in (3.24) below that

$$
\sum_{i=1}^{n}\left(\mu_{i}(t)\right)^{p} \sum_{i=1}^{n}\left(\mu_{i}(t)\right)^{2(1-\beta)} \geq 1
$$

and since we specifically chose $p=2 \beta$ we have also

$$
\sum_{i=1}^{n}\left(\mu_{i}(t)\right)^{p} \sum_{i=1}^{n}\left(\mu_{i}(t)\right)^{1-2 \beta} \leq \frac{n}{n^{p}} \cdot \frac{n}{n^{1-2 \beta}}=n
$$

which completes the argument. (Notice that diversity-weighted portfolios are defined for $p \in(0,1)$ only, therefore we needed the condition $0<\beta<1 / 2$ in order to be able to choose $p=2 \beta$ and still have $p \in(0,1)$. )

\subsubsection{Generalized excess growth rate of the market portfolio}

We can construct a similar example of an arbitrage that is valid for any value of $\beta>0$ using the notion of generalized excess growth rate and Proposition 3.8 in Fernholz and Karatzas (2005).

Notice that in model (3.16) we have $s_{\mu \nu}(t)=\sum_{i=1}^{n} \mu_{i}(t) s_{i \nu}(t)=\sigma\left(\mu_{\nu}(t)\right)^{1-\beta}$ for the quantities of (3.7), and

$$
\begin{aligned}
\tau_{i i}^{\mu}(t) & =\sum_{\nu=1}^{n}\left(s_{i \nu}(t)-s_{\mu \nu}(t)\right)^{2}=\sum_{\nu \neq i}\left(s_{\mu \nu}(t)\right)^{2}+\left(s_{i i}(t)-s_{\mu i}(t)\right)^{2} \\
& =\sigma^{2} \sum_{\nu \neq i}\left(\mu_{\nu}(t)\right)^{2(1-\beta)}+\sigma^{2}\left(\mu_{i}(t)\right)^{-2 \beta}\left(1-\mu_{i}(t)\right)^{2} \\
& =\sigma^{2} \sum_{\nu=1}^{n}\left(\mu_{\nu}(t)\right)^{2(1-\beta)}+\sigma^{2}\left(\mu_{i}(t)\right)^{-2 \beta}\left(1-2 \mu_{i}(t)\right)
\end{aligned}
$$

for those of (3.11). Therefore, the generalized excess growth rate

$$
\gamma_{\mu, p}^{*}(t):=\frac{1}{2} \sum_{i=1}^{n}\left(\mu_{i}(t)\right)^{p} \tau_{i i}^{\mu}(t), \quad 0<p<1
$$


for this market, introduced in (3.24) of Fernholz and Karatzas (2005), now takes the form

$$
\frac{2}{\sigma^{2}} \gamma_{\mu, p}^{*}(t)=\sum_{i=1}^{n}\left(\mu_{i}(t)\right)^{p} \cdot \sum_{\nu=1}^{n}\left(\mu_{\nu}(t)\right)^{2(1-\beta)}+\sum_{i=1}^{n}\left(\mu_{i}(t)\right)^{p-2 \beta}\left(1-2 \mu_{i}(t)\right) .
$$

Assume now that we choose $p \in(0,1)$ so that $0<p \leq 2 \beta$. Then we also have $2(1-\beta) \leq 2-p$, and since obviously $\mu_{i}(t)<1$, we also have $\left(\mu_{i}(t)\right)^{2(1-\beta)} \geq\left(\mu_{i}(t)\right)^{2-p}$ for all $i=1, \ldots, n$ and all $t$. Thus, for the first term on the right-hand side in (3.23) we have

$$
\sum_{i=1}^{n}\left(\mu_{i}(t)\right)^{p} \cdot \sum_{\nu=1}^{n}\left(\mu_{\nu}(t)\right)^{2(1-\beta)} \geq \sum_{i=1}^{n}\left(\mu_{i}(t)\right)^{p} \cdot \sum_{i=1}^{n}\left(\mu_{i}(t)\right)^{2-p} \geq 1,
$$

where the last inequality follows from Cauchy-Schwarz, namely

$$
1=\sum_{i=1}^{n}\left(\mu_{i}(t)\right)^{\frac{p}{2}} \cdot\left(\mu_{i}(t)\right)^{1-\frac{p}{2}} \leq\left(\sum_{i=1}^{n}\left(\mu_{i}(t)\right)^{p} \cdot \sum_{i=1}^{n}\left(\mu_{i}(t)\right)^{2-p}\right)^{\frac{1}{2}}
$$

For the second term on the right-hand side in (3.23) and for every fixed $t$, we need to consider two cases:

First, if all market weights are smaller than $1 / 2$, i.e., $0<\mu_{i}(t) \leq 1 / 2$ for $i=1, \ldots, n$, then we have $\left(\mu_{i}(t)\right)^{p-2 \beta} \geq(1 / 2)^{p-2 \beta}=2^{2 \beta-p} \geq 1$, therefore also

$$
\sum_{i=1}^{n}\left(\mu_{i}(t)\right)^{p-2 \beta}\left(1-2 \mu_{i}(t)\right) \geq \sum_{i=1}^{n} 2^{2 \beta-p}\left(1-2 \mu_{i}(t)\right)=2^{2 \beta-p}(n-2) \geq n-2 .
$$

Secondly, if one of the market weights is bigger than $1 / 2$, i.e., there exist an integer $1 \leq j \leq n$ such that $1 / 2<\mu_{j}(t) \leq 1$, the remaining market weights must then all be strictly less than $1 / 2$, i.e., $0<\mu_{i}(t)<1 / 2$ for $i \neq j$. In this case we have $1 \leq\left(\mu_{j}(t)\right)^{p-2 \beta} \leq 2^{2 \beta-p}$, and $\left(\mu_{i}(t)\right)^{p-2 \beta} \geq 2^{2 \beta-p}$ for $i \neq j$, and moreover we have $-1 \leq\left(1-2 \mu_{j}(t)\right)<0$, and $\left(1-2 \mu_{i}(t)\right)>0$ for $i \neq j$. We obtain

$$
\sum_{i=1}^{n}\left(\mu_{i}(t)\right)^{p-2 \beta}\left(1-2 \mu_{i}(t)\right)=\left(\mu_{j}(t)\right)^{p-2 \beta}\left(1-2 \mu_{j}(t)\right)+\sum_{i \neq j}\left(\mu_{i}(t)\right)^{p-2 \beta}\left(1-2 \mu_{i}(t)\right)
$$


Chapter 3. Generalized Volatility-Stabilized processes in Stochastic Portfolio Theory

$$
\begin{aligned}
& \geq-2^{2 \beta-p}+\sum_{i \neq j} 2^{2 \beta-p}\left(1-2 \mu_{i}(t)\right)=2^{2 \beta-p}\left[-1+(n-1)-2\left(1-\mu_{j}(t)\right)\right] \\
& =2^{2 \beta-p}\left(n-4+2 \mu_{j}(t)\right)>2^{2 \beta-p}(n-3)>n-3
\end{aligned}
$$

Thus, in either case, the second term on the right-hand side of (3.23) satisfies

$$
\sum_{i=1}^{n}\left(\mu_{i}(t)\right)^{p-2 \beta}\left(1-2 \mu_{i}(t)\right)>n-3 .
$$

If we combine (3.23), (3.24) and (3.25) together, still under the assumption that $0<p \leq 2 \beta$, we obtain

$$
\frac{2}{\sigma^{2}} \gamma_{\mu, p}^{*}(t)>1+n-3=n-2,
$$

and $\gamma_{\mu, p}^{*}(t)>0$ for $n \geq 2$ and all $t$. Now, Proposition 3.8 in Fernholz and Karatzas (2005) guarantees that, over sufficiently long time-horizons $[0, T]$ (in particular, with

$\left.T>\frac{2}{p \sigma^{2}} \cdot \frac{\log (n) \cdot n^{1-p}}{n-2}\right)$, there exist arbitrages relative to the market portfolio $\mu(\cdot)$. More precisely, it is shown there that the portfolio rule

$$
\pi_{i}(t)=p \frac{\left(\mu_{i}(t)\right)^{p}}{\sum_{j=1}^{n}\left(\mu_{j}(t)\right)^{p}}+(1-p) \mu_{i}(t), \quad i=1, \ldots n
$$

is a strong arbitrage opportunity relative to the market portfolio $\mu(t)$ in the sense of definition (3.13).

Notice that the portfolio of (3.27) is a convex combination, with fixed weights $1-p$ and $p$, of the market portfolio and of the diversity-weighted portfolio of (3.19), respectively. Note also that if $\beta \geq 1 / 2$ one can choose $p \in(0,1)$ arbitrarily, but if $0<\beta<1 / 2$ one needs to choose $p \in(0,2 \beta]$, in order to get the inequality in (3.24).

\subsubsection{Short-term Arbitrage in the General Model}

Let us return to the general model of (2.1), in which the stocks' volatilities are given by

$$
s_{i \nu}(t)=\sigma\left(\mu_{i}(t)\right)^{-\beta} K(X(t)) \delta_{i \nu},
$$


therefore we have

$$
s_{\mu \nu}(t)=\sum_{i=1}^{n} \mu_{i}(t) s_{i \nu}(t)=\sigma\left(\mu_{\nu}(t)\right)^{1-\beta} K(X(t))
$$

and the variance relative to the market of the $i$ th stock is

$$
\begin{aligned}
\tau_{i i}^{\mu}(t) & =\sum_{\nu=1}^{n}\left(s_{i \nu}(t)-s_{\mu \nu}(t)\right)^{2}=\sum_{\nu \neq i}\left(s_{\mu \nu}(t)\right)^{2}+\left(s_{i i}(t)-s_{\mu i}(t)\right)^{2} \\
& =\sigma^{2}[K(X(t))]^{2}\left[\sum_{\nu=1}^{n}\left(\mu_{\nu}(t)\right)^{2(1-\beta)}+\left(\mu_{i}(t)\right)^{-2 \beta}\left(1-2 \mu_{i}(t)\right)\right] .
\end{aligned}
$$

Consider the case of $K(\cdot)$ bounded away from zero, i.e., there exists $K_{\min }>0$ such that $K_{\min } \leq K(u)$ for all $u \in(0, \infty)^{n}$. Then the excess growth rate of the market portfolio $\mu(\cdot)$ is bounded away from zero as well, namely

$$
\gamma_{\mu}^{*}(t) \geq \frac{\sigma^{2}}{2}(n-1)[K(\mu(t))]^{2} \geq \frac{\sigma^{2}}{2}(n-1) K_{\min }^{2}>0
$$

whenever $\beta \in[1 / 2, \infty)$, thanks to $(3.18)$.

Moreover, for any value of $\beta \in(0, \infty)$ and arbitrary $p \leq 2 \beta$, the excess growth rate of the diversity-weighted portfolio, defined in (3.19), is bounded away from zero. Indeed, using the inequality in (3.20), we obtain

$$
\gamma_{\mu(p)}^{*}(t) \geq \frac{\sigma^{2}}{2} \frac{n-1}{n^{1-p}}[K(X(t))]^{2} \geq \frac{\sigma^{2}}{2} \frac{n-1}{n^{1-p}} K_{\min }^{2}>0 .
$$

Therefore, recalling the formula in (3.21), and the computations in (3.22), there exist strong arbitrage opportunities relative to the market portfolio over sufficiently large time horizons for any value of $\beta>0$. For instance, with $p \in(0, \min \{1,2 \beta\})$ and

$$
T>\frac{2}{p \sigma^{2} K_{\min }^{2}} \cdot \frac{\log (n) \cdot n^{1-p}}{n-1}=: T^{*}\left(\beta, \sigma, n, K_{\min }\right)
$$

the diversity-weighted portfolio $\mu^{(p)}(\cdot)$ outperforms the market over $[0, T]$.

If in addition to the assumption that $K(\cdot)$ is bounded away from zero, we assume that $\beta \geq 1 / 2$, then we obtain from (3.28) a lower bound on the individual 
stocks' covariances relative to the market portfolio $\mu(\cdot)$, namely

$$
\tau_{i i}^{\mu}(t) \geq \sigma^{2} K_{\min }^{2}\left(\frac{1}{\mu_{i}(t)}-1\right)
$$

This allows to use the same approach as in Proposition 2 in Section 5 of Banner and Fernholz (2008), and construct a portfolio which is guaranteed to outperform the market portfolio over arbitrarily short time horizon ("short-term arbitrage"). Note, that if $\sigma^{2} K_{\min }^{2} \geq 1$, then we can use exactly the same construction (and the same portfolio rule) as in Proposition 2 in Section 5 of Banner and Fernholz (2008). If $\sigma^{2} K_{\min }^{2}<1$, then only minor adjustments are needed. Hence, if $K(\cdot)$ is bounded and $\beta \geq 1 / 2$, then short-term arbitrage exists in the model of (2.1).

We summarize the results of this section in the following proposition.

Proposition 3.1. If the function $K(\cdot)$ is bounded away from zero, then there exist strong long-term arbitrage opportunities in the financial market with stocks' capitalizations following the system in (2.1). If in addition $\beta \geq 1 / 2$, then strong short-term arbitrage is present in this market.

Example: Let us conclude with a simple example of systems that lead to markets in which both long-term and short-term arbitrage opportunities are present. It is easy to see that if $K(\cdot)$ is chosen to be the reciprocal of the $L_{p}$-norm of the market weights (defined in (2.2)), with $p \geq 1$, then $K(\cdot)$ is bounded on the state space and has locally bounded partial derivatives. Therefore, the corresponding system of stochastic differential equations in (2.1), which with this choice takes the form

$$
\begin{aligned}
\mathrm{d}\left(\log X_{i}(t)\right) & =\frac{\alpha_{i}}{2\left(\mu_{i}(t)\right)^{2 \beta}\|\mu(t)\|_{p}^{2}} \mathrm{~d} t+\frac{\sigma}{\left(\mu_{i}(t)\right)^{\beta}\|\mu(t)\|_{p}} \mathrm{~d} W_{i}(t),(3.2, n \\
X_{i}(0) & =x_{i}>0, \quad i=1, \ldots, n
\end{aligned}
$$

has a unique in distribution weak solution, and it also admits a pathwise unique, strong solution. Moreover, according to the results obove, if $\beta \geq 1 / 2$, then shortterm (as well as long-term) arbitrage opportunities exist in the model described by 
Chapter 3. Generalized Volatility-Stabilized processes in Stochastic Portfolio Theory

Remark: Assuming that the function $K(\cdot)$ is bounded and $\beta \leq 1 / 2$, one can use the so-called "market price of risk" process to compute the corresponding exponential local martingale (for definitions and more details, see Remark 2.1 in Fernholz and Karatzas (2005)), and express it as follows:

$L(T)=\left\{\prod_{i=1}^{n}\left[\frac{X_{i}(0)}{X_{i}(T)}\right]^{p_{i}}\right\} \exp \left\{-\frac{1}{2} \int_{0}^{T} K(X(t)) \sum_{i=1}^{n}\left[\sigma^{2} p_{i}-\frac{2}{\alpha_{i}} \mathrm{I}_{\left[\alpha_{i}>0\right]}\right] \frac{p_{i} \mathrm{~d} t}{\left(\mu_{i}(t)\right)^{2 \beta}}\right\}$

where $p_{i}=\frac{1}{2}\left[1+\frac{\alpha_{i}}{\sigma^{2}}\right]$. This process is in fact strict local martingale, according to the Proposition 3.4 in Fernholz and Karatzas (2005). Indeed, there are arbitrage opportunities present in the market models under consideration, in particular, we showed that a long-only portfolio (diversity-weighted portfolio) is an arbitrage opportunity relative to another long only portfolio (the market portfolio), hence, it is obvious that there cannot exist an equivalent martingale measure.

\subsection{Market Weights and the Diversity Weighted Portfolio}

Let us consider in this section the case of the system in $(2.1)$ with $K(\cdot) \equiv 1$, that is, we are considering the following system of stochastic differential equations

$$
\begin{aligned}
\mathrm{d}\left(\log X_{i}(t)\right) & =\frac{\alpha_{i}}{2\left(\mu_{i}(t)\right)^{2 \beta}} \mathrm{d} t+\frac{\sigma}{\left(\mu_{i}(t)\right)^{\beta}} \mathrm{d} W_{i}(t), \\
X_{i}(0) & =x_{i}>0, \quad i=1, \ldots, n
\end{aligned}
$$

where $\beta>0, \alpha_{i} \geq 0, \sigma>0$,

$$
\mu_{i}(t)=\frac{X_{i}(t)}{S(t)}=\frac{X_{i}(t)}{X_{1}(t)+\ldots+X_{n}(t)},
$$

and $W(\cdot)=\left(W_{1}(\cdot), \ldots, W_{n}(\cdot)\right)$ is an $n$-dimensional Brownian motion with an integer $n \geq 2$. 
Chapter 3. Generalized Volatility-Stabilized processes in Stochastic Portfolio Theory

\subsubsection{Dynamics of the Market Weights under the GVSM}

The system in (3.30) is equivalent to the following system of stochastic differential equations

$$
\mathrm{d} X_{i}(t)=\frac{\alpha_{i}+\sigma^{2}}{2}\left(X_{i}(t)\right)^{1-2 \beta}(S(t))^{2 \beta} \mathrm{d} t+\sigma\left(X_{i}(t)\right)^{1-\beta}(S(t))^{\beta} \mathrm{d} W_{i}(t)
$$

for each $i=1, \ldots, n$. Then, for the strictly positive process $S(\cdot)=X_{1}(\cdot)+\ldots+X_{n}(\cdot)$ of the total capitalization of the market we have the following dynamics

$$
\begin{aligned}
\mathrm{d} S(t)=(S(t))^{2 \beta} & \left(\sum_{\nu=1}^{n} \frac{\alpha_{\nu}+\sigma^{2}}{2}\left(X_{\nu}(t)\right)^{1-2 \beta}\right) \mathrm{d} t \\
& +\sigma(S(t))^{\beta}\left(\sum_{\nu=1}^{n}\left(X_{\nu}(t)\right)^{1-\beta} \mathrm{d} W_{\nu}(t)\right) .
\end{aligned}
$$

Notice that

$$
\mathrm{d}\langle S\rangle(t)=\sigma^{2}(S(t))^{2 \beta}\left(\sum_{\nu=1}^{n}\left(X_{\nu}(t)\right)^{2-2 \beta}\right) \mathrm{d} t,
$$

and

$$
\mathrm{d}\left\langle X_{i}, S\right\rangle(t)=\sigma^{2}(S(t))^{2 \beta}\left(X_{i}(t)\right)^{2-2 \beta} \mathrm{d} t .
$$

Direct application of multidimensional Itô's rule, where the function $f(u, v)=u / v$ maps $(0, \infty) \times(0, \infty)$ onto $(0, \infty)$, gives the dynamics for the market weights as follows

$$
\begin{aligned}
& \mathrm{d} \mu_{i}(t)=\mathrm{d} f\left(X_{i}(t), S(t)\right)= \\
& =\frac{1}{S(t)} \mathrm{d} X_{i}(t)-\frac{X_{i}(t)}{(S(t))^{2}} \mathrm{~d} S(t)+\frac{1}{2} \frac{2 X_{i}(t)}{(S(t))^{3}} \mathrm{~d}\langle S\rangle(t)-\frac{1}{(S(t))^{2}} \mathrm{~d}\left\langle X_{i}, S\right\rangle(t) .
\end{aligned}
$$

Hence, recalling (3.31), (3.32), and substituting $\mu_{i}(t)=X_{i}(t) / S(t)$, we obtain the stochastic differential equation for $\mu_{i}(\cdot)$, namely

$$
\mathrm{d} \mu_{i}(t)=\frac{1}{2}\left[\left(\mu_{i}(t)\right)^{1-2 \beta}\left(\alpha_{i}+\sigma^{2}\left(1-2 \mu_{i}(t)\right)\right)\right.
$$


Chapter 3. Generalized Volatility-Stabilized processes in Stochastic Portfolio Theory

$$
\begin{array}{r}
\left.-\mu_{i}(t)\left(\sum_{\nu=1}^{n}\left(\mu_{\nu}(t)\right)^{1-2 \beta}\left(\alpha_{\nu}+\sigma^{2}\left(1-2 \mu_{\nu}(t)\right)\right)\right)\right] \mathrm{d} t \\
+\sigma\left(\mu_{i}(t)\right)^{1-\beta} \mathrm{d} W_{i}(t)-\sigma \mu_{i}(t)\left(\sum_{\nu=1}^{n}\left(\mu_{\nu}(t)\right)^{1-\beta} \mathrm{d} W_{\nu}(t)\right) .
\end{array}
$$

Note (as a sanity check) that the sum over $i=1, \ldots, n$ of both the drift and diffusion terms in this equation is zero, as it should for $\mathrm{d} \mu_{1}(t)+\cdots+\mathrm{d} \mu_{n}(t)=\mathrm{d}\left(\mu_{1}(t)+\right.$ $\left.\cdots+\mu_{n}(t)\right)=0$. The stochastic differential equation for $\mu_{i}(\cdot)$ can equivalently be written as

$$
\begin{aligned}
\frac{\mathrm{d} \mu_{i}(t)}{\mu_{i}(t)}=\frac{1}{2}\left[\left(\mu_{i}(t)\right)^{-2 \beta}\left(\alpha_{i}+\sigma^{2}\left(1-2 \mu_{i}(t)\right)\right)\right. & \\
& \left.-\left(\sum_{\nu=1}^{n}\left(\mu_{\nu}(t)\right)^{1-2 \beta}\left(\alpha_{\nu}+\sigma^{2}\left(1-2 \mu_{\nu}(t)\right)\right)\right)\right] \mathrm{d} t \\
+ & \sigma\left(\mu_{i}(t)\right)^{-\beta}\left(1-\mu_{i}(t)\right) \mathrm{d} W_{i}(t)-\sigma\left(\sum_{\nu \neq i}\left(\mu_{\nu}(t)\right)^{1-\beta} \mathrm{d} W_{\nu}(t)\right),
\end{aligned}
$$

which is an equation for a diffusion process with values in the strictly positive simplex.

From (3.34), it is now easy to derive the variance-covariance structure of the system of market weights, in particular for $i \neq j$ we have

$$
\begin{aligned}
\mathrm{d}\left\langle\mu_{i}, \mu_{j}\right\rangle(t)=-\sigma^{2}( & \left.\mu_{i}(t)\right)^{2-2 \beta}\left(1-\mu_{i}(t)\right) \mu_{j}(t) \mathrm{d}\left\langle W_{i}\right\rangle(t) \\
& -\sigma^{2}\left(\mu_{j}(t)\right)^{2-2 \beta}\left(1-\mu_{j}(t)\right) \mu_{i}(t) \mathrm{d}\left\langle W_{j}\right\rangle(t) \\
& +\sigma^{2} \mu_{i}(t) \mu_{j}(t)\left(\sum_{\nu \neq i, j}\left(\mu_{\nu}(t)\right)^{2(1-\beta)} \mathrm{d}\left\langle W_{\nu}\right\rangle(t)\right),
\end{aligned}
$$

which can be simplified as

$$
\begin{aligned}
\frac{\mathrm{d}\left\langle\mu_{i}, \mu_{j}\right\rangle(t)}{\sigma^{2} \mathrm{~d} t}=\mu_{i}(t) & \mu_{j}(t)\left(\sum_{\nu=1}^{n}\left(\mu_{\nu}(t)\right)^{2(1-\beta)}\right) \\
& -\mu_{i}(t) \mu_{j}(t)\left[\left(\mu_{i}(t)\right)^{1-2 \beta}+\left(\mu_{j}(t)\right)^{1-2 \beta}\right],
\end{aligned}
$$

and for $i=1, \ldots, n$ we have

$$
\frac{\mathrm{d}\left\langle\mu_{i}\right\rangle(t)}{\sigma^{2} \mathrm{~d} t}=\left(\mu_{i}(t)\right)^{2}\left[\sum_{\nu=1}^{n}\left(\mu_{\nu}(t)\right)^{2(1-\beta)}+\left(\mu_{i}(t)\right)^{-2 \beta}\left(1-2 \mu_{i}(t)\right)\right] .
$$


Remark: Note that in the special case of $\beta=1 / 2, \alpha_{1}=\ldots=\alpha_{n}=\alpha \geq 0$ and $\sigma=1$, the equations in (3.33) simplify as follows

$$
\begin{aligned}
\mathrm{d} \mu_{i}(t)= & \frac{1}{2}\left[\left(\alpha+\left(1-2 \mu_{i}(t)\right)\right)-\mu_{i}(t)\left(\sum_{\nu=1}^{n}\left(\alpha+\left(1-2 \mu_{\nu}(t)\right)\right)\right)\right] \mathrm{d} t \\
& +\left(\mu_{i}(t)\right)^{1 / 2} \mathrm{~d} W_{i}(t)-\mu_{i}(t)\left(\sum_{\nu=1}^{n}\left(\mu_{\nu}(t)\right)^{1 / 2} \mathrm{~d} W_{\nu}(t)\right) \\
= & \frac{1}{2}(\alpha+1)\left[1-n \mu_{i}(t)\right] \mathrm{d} t+\sqrt{\mu_{i}(t)}\left(\sum_{\nu=1}^{n}\left(\delta_{i \nu}-\sqrt{\mu_{i}(t) \mu_{\nu}(t)}\right) \mathrm{d} W_{\nu}(t)\right),
\end{aligned}
$$

and from (3.35)

$$
\frac{\mathrm{d}\left\langle\mu_{i}\right\rangle(t)}{\mathrm{d} t}=\mu_{i}(t)\left(1-\mu_{i}(t)\right)
$$

As was shown already in Goia (2009) and Pal (2011), this system of stochastic differential equations belongs to the family of Wright-Fisher diffusions

$$
\mathrm{d} \mu_{i}(t)=\frac{1}{2}(\alpha+1)\left[1-n \mu_{i}(t)\right] \mathrm{d} t+\sqrt{\mu_{i}(t)\left(1-\mu_{i}(t)\right)} \mathrm{d} \widehat{W}_{i}(t)
$$

where the processes $\widehat{W}_{1}, \ldots, \widehat{W}_{n}$ defined as

$$
\widehat{W}_{i}(t) \triangleq \int_{0}^{t} \sum_{\nu=1}^{n} \frac{\delta_{i \nu}-\sqrt{\mu_{i}(t) \mu_{\nu}(t)}}{\sqrt{1-\mu_{i}(t)}} \mathrm{d} W_{\nu}(s), \quad 0 \leq t<\infty
$$

are standard, but dependent, Brownian motions by P. Lévy's theorem (see for instance Theorem 3.16 in Karatzas and Shreve (1991)). Since for the case of $n=2$, the first coordinate of the Wright-Fisher diffusion is also known as the Jacobi diffusion, the general class is also referred to as the multidimensional Jacobi diffusions (see Goia (2009)).

Thus in the case $\beta=1 / 2, \alpha_{1}=\ldots=\alpha_{n}=\alpha \geq 0$ and $\sigma=1$, not only is $\left(\mu_{1}(\cdot), \ldots, \mu_{n}(\cdot)\right)$ a diffusion with values in the positive simplex, but each one of the components is a Wright-Fisher diffusion of the form $(3.36)$ in the interval $(0,1)$ in its own right. 
Chapter 3. Generalized Volatility-Stabilized processes in Stochastic Portfolio Theory

\subsubsection{Dynamics of the Weights of the Diversity Weighted Portfolio under the GVSM}

We will now derive the dynamics for the weights of the diversity weighted portfolio

$$
\mu_{i}^{(p)}(t) \triangleq \frac{\left(X_{i}(t)\right)^{p}}{S^{(p)}(t)}
$$

where we have defined

$$
S^{(p)}(t) \triangleq\left(X_{1}(t)\right)^{p}+\ldots+\left(X_{n}(t)\right)^{p}
$$

and $p \in(0,1]$. For the special case of $p=1$ we will continue using the notation

$$
S(t)=S^{(1)}(t)=X_{1}(t)+\ldots+X_{n}(t),
$$

for the process solving the stochastic differential equation in (3.32). We will in particular be interested in the case of $p=2 \beta$ for $\beta \in(0,1 / 2]$.

From (3.31) one can easily derive, using Itô's rule, the dynamics for the strictly positive processes $\left(X_{i}(\cdot)\right)^{2 \beta}$ as follows

$$
\begin{aligned}
\mathrm{d}\left(X_{i}(t)\right)^{2 \beta} & =2 \beta\left(X_{i}(t)\right)^{2 \beta-1} \mathrm{~d} X_{i}(t)+\frac{1}{2} 2 \beta(2 \beta-1)\left(X_{i}(t)\right)^{2 \beta-2} \mathrm{~d}\left\langle X_{i}\right\rangle(t) \\
& =\beta\left(\alpha_{i}+2 \beta \sigma^{2}\right)(S(t))^{2 \beta} \mathrm{d} t+2 \beta \sigma\left(X_{i}(t)\right)^{\beta}(S(t))^{\beta} \mathrm{d} W_{i}(t) .
\end{aligned}
$$

Therefore the process $S^{(2 \beta)}(\cdot)$ solves the following stochastic differential equation

$$
\mathrm{d} S^{(2 \beta)}(t)=\beta\left(\sum_{\nu=1}^{n} \alpha_{\nu}+2 n \beta \sigma^{2}\right)(S(t))^{2 \beta} \mathrm{d} t+2 \beta \sigma(S(t))^{\beta} \sqrt{S^{(2 \beta)}(t)} \mathrm{d} B(t),
$$

where

$$
B(t) \triangleq \int_{0}^{t} \sum_{\nu=1}^{n} \sqrt{\frac{\left(X_{\nu}(s)\right)^{2 \beta}}{S^{(2 \beta)}(s)}} \mathrm{d} W_{\nu}(s), \quad 0 \leq t<\infty
$$

is a standard Brownian motion by P. Lévy's theorem (see for instance Theorem 3.16 in Karatzas and Shreve (1991)). 
Remark: Note that in the special case of $\beta=1 / 2$, the process $S(\cdot)$, as in (3.32), solves the following stochastic differential equation

$$
\mathrm{d} S(t)=\frac{1}{2}\left(\sum_{\nu=1}^{n} \alpha_{\nu}+n \sigma^{2}\right) S(t) \mathrm{d} t+\sigma(S(t))^{1 / 2}\left(\sum_{\nu=1}^{n}\left(X_{\nu}(t)\right)^{1 / 2} \mathrm{~d} W_{\nu}(t)\right),
$$

which can be written as

$$
\mathrm{d} S(t)=\frac{1}{2}\left(\sum_{\nu=1}^{n} \alpha_{\nu}+n \sigma^{2}\right) S(t) \mathrm{d} t+\sigma S(t) \mathrm{d} \tilde{B}(t),
$$

where

$$
\tilde{B}(t) \triangleq \int_{0}^{t} \sum_{\nu=1}^{n} \sqrt{\frac{X_{\nu}(s)}{S(s)}} \mathrm{d} W_{\nu}(s), \quad 0 \leq t<\infty
$$

is another standard Brownian motion by P. Lévy's theorem (see for instance Theorem 3.16 in Karatzas and Shreve (1991)). In other words, in this case the process $S(\cdot)$ is a geometric Brownian motion, as was already observed in Fernholz and Karatzas (2005).

In order to derive the dynamics for the weights of the diversity weighted portfolio $\mu_{i}^{(2 \beta)}(t)$ we will again apply multidimensional Itô's rule, with the function $f(u, v)=u / v$, as follows

$$
\begin{aligned}
\mathrm{d} \mu_{i}^{(2 \beta)}(t)= & \frac{1}{S^{(2 \beta)}(t)} \mathrm{d}\left(X_{i}(t)\right)^{2 \beta}-\frac{\left(X_{i}(t)\right)^{2 \beta}}{\left(S^{(2 \beta)}(t)\right)^{2}} \mathrm{~d} S^{(2 \beta)}(t) \\
& +\frac{1}{2} \frac{2\left(X_{i}(t)\right)^{2 \beta}}{\left(S^{(2 \beta)}(t)\right)^{3}} \mathrm{~d}\left\langle S^{(2 \beta)}\right\rangle(t)-\frac{1}{\left(S^{(2 \beta)}(t)\right)^{2}} \mathrm{~d}\left\langle\left(X_{i}(t)\right)^{2 \beta}, S^{(2 \beta)}\right\rangle(t)
\end{aligned}
$$

Recalling (3.37), (3.37) and noticing that

$$
\mathrm{d}\left\langle S^{(2 \beta)}\right\rangle(t)=4 \beta^{2} \sigma^{2}(S(t))^{2 \beta} S^{(2 \beta)}(t) \mathrm{d} t
$$

and

$$
\mathrm{d}\left\langle\left(X_{i}\right)^{2 \beta}, S^{(2 \beta)}\right\rangle(t)=4 \beta^{2} \sigma^{2}(S(t))^{2 \beta}\left(X_{i}(t)\right)^{2 \beta} \mathrm{d} t,
$$


Chapter 3. Generalized Volatility-Stabilized processes in Stochastic Portfolio Theory

we further obtain

$$
\begin{aligned}
\mathrm{d} \mu_{i}^{(2 \beta)}(t)=\beta\left[\alpha_{i}\right. & \left.+2 \beta \sigma^{2}-\left(\sum_{\nu=1}^{n} \alpha_{\nu}+2 n \beta \sigma^{2}\right) \mu_{i}^{(2 \beta)}(t)\right] \frac{(S(t))^{2 \beta}}{S^{(2 \beta)}(t)} \mathrm{d} t \\
& +2 \beta \sigma \frac{(S(t))^{\beta}}{S^{(2 \beta)}(t)}\left[\left(X_{i}(t)\right)^{\beta} \mathrm{d} W_{i}(t)-\mu_{i}^{(2 \beta)}(t) \sum_{\nu=1}^{n}\left(X_{\nu}(t)\right)^{\beta} \mathrm{d} W_{\nu}(t)\right] .
\end{aligned}
$$

Thus, the stochastic differential equation for $\mu_{i}^{(2 \beta)}(\cdot)$ is

$$
\begin{aligned}
\mathrm{d} \mu_{i}^{(2 \beta)}(t)= & \beta\left[\alpha_{i}+2 \beta \sigma^{2}-\left(\sum_{\nu=1}^{n} \alpha_{\nu}+2 n \beta \sigma^{2}\right) \mu_{i}^{(2 \beta)}(t)\right] \frac{(S(t))^{2 \beta}}{S^{(2 \beta)}(t)} \mathrm{d} t \\
& +2 \beta \sigma \frac{(S(t))^{\beta}}{\sqrt{S^{(2 \beta)}(t)}}\left[\sqrt{\mu_{i}^{(2 \beta)}(t)} \sum_{\nu=1}^{n}\left(\delta_{i \nu}-\sqrt{\mu_{i}^{(2 \beta)}(t) \mu_{\nu}^{(2 \beta)}(t)}\right) \mathrm{d} W_{\nu}(t)\right] .
\end{aligned}
$$

From (3.38), it is easy to derive the variance-covariance structure of the system of the weights $\mu_{i}^{(2 \beta)}(\cdot)$, in particular for $i \neq j$ we have

$$
\begin{aligned}
& \mathrm{d}\left\langle\mu_{i}^{(2 \beta)}, \mu_{j}^{(2 \beta)}\right\rangle(t)= \\
& =\left(2 \beta \sigma \frac{(S(t))^{\beta}}{\sqrt{S^{(2 \beta)}(t)}}\right)^{2}\left[\sqrt{\mu_{i}^{(2 \beta)}(t)}\left(1-\mu_{i}^{(2 \beta)}(t)\right)\left(-\mu_{j}^{(2 \beta)}(t) \sqrt{\mu_{i}^{(2 \beta)}(t)}\right) \mathrm{d}\left\langle W_{i}\right\rangle(t)\right. \\
& \quad+\sqrt{\mu_{j}^{(2 \beta)}(t)}\left(1-\mu_{j}^{(2 \beta)}(t)\right)\left(-\mu_{i}^{(2 \beta)}(t) \sqrt{\mu_{j}^{(2 \beta)}(t)}\right) \mathrm{d}\left\langle W_{j}\right\rangle(t) \\
& \left.\quad+\mu_{i}^{(2 \beta)}(t) \mu_{j}^{(2 \beta)}(t) \sum_{\nu \neq i, j} \mu_{\nu}^{(2 \beta)}(t) \mathrm{d}\left\langle W_{\nu}\right\rangle(t)\right],
\end{aligned}
$$

which can further be simplified as

$$
\begin{aligned}
& \mathrm{d}\left\langle\mu_{i}^{(2 \beta)}, \mu_{j}^{(2 \beta)}\right\rangle(t)= \\
& =4 \beta^{2} \sigma^{2} \frac{(S(t))^{2 \beta}}{S^{(2 \beta)}(t)}\left[\mu_{i}^{(2 \beta)}(t) \mu_{j}^{(2 \beta)}(t) \sum_{\nu=1}^{n} \mu_{\nu}^{(2 \beta)}(t)-2 \mu_{i}^{(2 \beta)}(t) \mu_{j}^{(2 \beta)}(t)\right] \mathrm{d} t \\
& =-4 \beta^{2} \sigma^{2} \frac{(S(t))^{2 \beta}}{S^{(2 \beta)}(t)} \mu_{i}^{(2 \beta)}(t) \mu_{j}^{(2 \beta)}(t) \mathrm{d} t .
\end{aligned}
$$

For $i=1, \ldots, n$ we have

$$
\mathrm{d}\left\langle\mu_{i}^{(2 \beta)}\right\rangle(t)=4 \beta^{2} \sigma^{2} \frac{(S(t))^{2 \beta}}{S^{(2 \beta)}(t)}\left[\left(\sqrt{\mu_{i}^{(2 \beta)}(t)}-\sqrt{\mu_{i}^{(2 \beta)}(t)} \mu_{i}^{(2 \beta)}(t)\right)^{2} \mathrm{~d}\left\langle W_{i}\right\rangle(t)\right.
$$


Chapter 3. Generalized Volatility-Stabilized processes in Stochastic Portfolio Theory

$$
\left.+\left(\mu_{i}^{(2 \beta)}(t)\right)^{2} \sum_{\nu \neq i} \mu_{\nu}^{(2 \beta)}(t) \mathrm{d}\left\langle W_{\nu}\right\rangle(t)\right]
$$

which simplifies to

$$
\mathrm{d}\left\langle\mu_{i}^{(2 \beta)}\right\rangle(t)=4 \beta^{2} \sigma^{2} \frac{(S(t))^{2 \beta}}{S^{(2 \beta)}(t)} \mu_{i}^{(2 \beta)}(t)\left(1-\mu_{i}^{(2 \beta)}(t)\right) \mathrm{d} t
$$

Similarly as before, choosing $\beta=1 / 2$ and $\sigma=1$ leads to the special case of Wright-Fisher diffusion as in (3.36). 


\section{Chapter 4}

\section{Asymptotics for the Generalized Volatility-Stabilized Processes}

Let us consider in this section the case of the system in (2.1) with

$$
K(\cdot) \equiv 1, \quad \text { and } \quad \alpha_{1}=\ldots=\alpha_{n}=\alpha \geq 0
$$

The assumption on equal drift coefficients is made here only in order to simplify the notation, the extension to the more general case is rather straightforward. In other words, we are assuming the following system of stochastic differential equations for the process $X(\cdot)=\left(X_{1}(\cdot), \ldots, X_{n}(\cdot)\right)$ of stocks' capitalizations

$$
\mathrm{d}\left(\log X_{i}(t)\right)=\frac{\alpha}{2\left(\mu_{i}(t)\right)^{2 \beta}} \mathrm{d} t+\frac{\sigma}{\left(\mu_{i}(t)\right)^{\beta}} \mathrm{d} W_{i}(t), \quad i=1, \ldots, n
$$

where $\beta>0$, and $\sigma>0$ are given real numbers.

We have constructed a weak solution of the system of stochastic differential equations (4.2), and argued that both uniqueness in distribution and pathwise uniqueness hold for this system, hence this solution is strong. Furthermore, using (2.65) and (2.66) we expressed the solution in terms of independent squaredBessel processes $Z_{i}(\cdot)$ which satisfy the equations in (2.54), (respectively, indepen- 
dent Bessel processes $R_{i}(\cdot)$ which satisfy the equations in (2.55)), namely:

$$
X_{i}(t)=(\beta \sigma)^{\frac{1}{\beta}}\left(Z_{i}(A(t))\right)^{\frac{1}{2 \beta}}=\left(\beta \sigma R_{i}(A(t))\right)^{\frac{1}{\beta}}, \quad 0 \leq t<\infty, i=1, \ldots, n .
$$

Note, that under the setting of this section, i.e. (4.1), all processes $Z_{i}(\cdot)$, as well as $R_{i}(\cdot)$, have "dimension" $m=m_{1}=\ldots=m_{n}=2+\frac{\alpha}{\beta \sigma^{2}}$. We will use the representations in (4.3) and the asymptotic properties known for squared-Bessel processes (respectively, for Bessel processes), to derive some asymptotic properties of the processes $X_{i}(\cdot)$, as well as the market weights $\mu_{i}(\cdot)$, following the model in (4.2).

Remark: Recall that we have used the representation (2.41) of the time-change process $\Upsilon(\cdot)$ defined in (2.32) in terms of squared-Bessel processes $Z_{i}(\cdot)$ of $(2.53)$, when we showed that $\Upsilon(\infty)=\infty$ a.s., see the inequality in (2.42). Note that we used the following asymptotic property

$$
\lim _{\theta \rightarrow \infty} \frac{1}{\log \theta} \int_{0}^{\theta} \frac{1}{Z(\xi)} \mathrm{d} \xi=\frac{1}{2 n-2}, \quad \text { a.s. }
$$

of the squared-Bessel process $Z(\cdot)=\sum_{j=1}^{n} Z_{j}(\cdot)$ in "dimension" $(2 . n)>2$. The statement (4.4) is a direct consequence of Lemma 4.2 in Cherny (2000); for completeness, we will establish it in subsection 4.3 .

In order to derive the asymptotic properties of the market under the model of (4.2), we will determine the asymptotic behavior of the time-change process $\Upsilon(\cdot)$ defined in (2.45) which can be expressed in terms of squared-Bessel processes $Z_{i}(\cdot)$ that satisfy the equations in (2.54), namely, assuming (4.1), the process $\Upsilon(\cdot)$ has the following representation

$$
\Upsilon(\theta)=(\beta \sigma)^{-2} \int_{0}^{\theta}\left(\sum_{j=1}^{n}\left(Z_{j}(\xi)\right)^{\frac{1}{2 \beta}}\right)^{-2 \beta} \mathrm{d} \xi, \quad 0 \leq \theta<\infty
$$


which is just a special case of (2.58).

In particular, we will prove in subsection 4.3 that process $\Upsilon(\cdot)$ of $(4.5)$ satisfies the following asymptotic property

$$
(\beta \sigma)^{2} \frac{\Upsilon(\theta)}{\log \theta}=\frac{1}{\log \theta} \int_{0}^{\theta} \frac{1}{\left(\sum_{j=1}^{n}\left(Z_{j}(\xi)\right)^{1 /(2 \beta)}\right)^{2 \beta}} \mathrm{d} \xi \underset{\theta / \infty}{\longrightarrow} \phi(1), \quad \text { a.s. }
$$

with the notation of

$$
\begin{aligned}
\phi(\theta) & \triangleq \int_{0}^{\infty} \ldots \int_{0}^{\infty}\left(\sum_{j=1}^{n}\left(q_{j}\right)^{1 /(2 \beta)}\right)^{-2 \beta} \frac{\left(q_{1} \ldots q_{n}\right)^{m / 2-1} \mathrm{e}^{-\left(q_{1}+\cdots+q_{n}\right) /(2 \theta)}}{\left((2 \theta)^{m / 2} \Gamma(m / 2)\right)^{n}} \mathrm{~d} q_{1} \ldots \mathrm{d} q_{n} \\
& =\frac{\phi(1)}{\theta} \in(0, \infty)
\end{aligned}
$$

for $0<\theta<\infty$, and with $\Gamma(\cdot)$ denoting the Gamma function. We shall also show in subsection 4.3 that this quantity is finite.

\subsection{Some Asymptotic Results - the Case of

$$
\alpha=0, \text { that is } m=2
$$

Considering the case when $m_{i}=m=2$, we know that 2-dimensional Bessel processes $R_{i}(\cdot)$ of $(2.56)$, satisfy the following (as was noted in Fernholz and Karatzas (2005))

$$
\lim _{\theta \rightarrow \infty} \frac{\log \left(R_{i}(\theta)\right)}{\log (\theta)}=\frac{1}{2} \quad \text { in probability, }
$$

and

$$
\limsup _{\theta \rightarrow \infty} \frac{\log \left(R_{i}(\theta)\right)}{\log (\theta)}=\frac{1}{2}, \quad \liminf _{\theta \rightarrow \infty} \frac{\log \left(R_{i}(\theta)\right)}{\log (\theta)}=-\infty, \quad \text { a.s. }
$$

Therefore, the process $X_{i}(\cdot)=\left(\beta \sigma R_{i}(A(\cdot))\right)^{\frac{1}{\beta}}$ satisfies

$$
\lim _{t \rightarrow \infty}\left(\frac{1}{t} \log \left(X_{i}(t)\right)\right)=\lim _{t \rightarrow \infty}\left(\frac{1}{t \beta} \log \left(\beta \sigma R_{i}(A(t))\right)\right)
$$




$$
\begin{aligned}
& =\frac{1}{\beta} \lim _{t \rightarrow \infty}\left(\frac{1}{t} \log \left(R_{i}(A(t))\right)\right)=\frac{1}{\beta} \lim _{t \rightarrow \infty}\left(\left.\frac{\log \left(R_{i}(\theta)\right) / \log \theta}{\Upsilon(\theta) / \log \theta}\right|_{\theta=A(t)}\right) \\
& =\frac{1}{\beta} \frac{1 / 2}{\phi(1) /(\beta \sigma)^{2}}=\frac{\beta \sigma^{2}}{2 \phi(1)} \quad \text { in probability }
\end{aligned}
$$

in the notation of (4.7), thanks to (4.6). Similarly,

$$
\limsup _{t \rightarrow \infty} \frac{1}{t} \log \left(X_{i}(t)\right)=\frac{\beta \sigma^{2}}{2 \phi(1)}, \text { and } \liminf _{\theta \rightarrow \infty} \frac{1}{t} \log \left(X_{i}(t)\right)=-\infty, \quad \text { a.s. }
$$

These results show that the market is not coherent (in the a.s. sense of Definition 2.1.1 in Fernholz (2002))when $\alpha=0$.

\subsection{Some Asymptotic Results - the Case of $\alpha>0$, that is $m>2$}

For the Bessel processes in "dimension" $m_{i}=m>2$ of (2.55) one can show that the asymptotic property (4.8) holds a.s., that is, we have

$$
\lim _{\theta \rightarrow \infty}\left(\frac{\log \left(R_{i}(\theta)\right)}{\log (\theta)}\right)=\frac{1}{2}, \quad \text { a.s. }
$$

(for the proof see for instance Fernholz and Karatzas (2005)). Therefore, for the process $X_{i}(t)=\left(\beta \sigma R_{i}(A(t))\right)^{\frac{1}{\beta}}$ we now obtain (using same derivation as in (4.9)) that (4.10) and (4.6) lead to

$\lim _{t \rightarrow \infty}\left(\frac{1}{t} \log \left(X_{i}(t)\right)\right)=\frac{1}{\beta} \lim _{t \rightarrow \infty}\left(\left.\frac{\log \left(R_{i}(\theta)\right) / \log \theta}{\Upsilon(\theta) / \log \theta}\right|_{\theta=A(t)}\right)=\frac{\beta \sigma^{2}}{2 \phi(1)}$,

thus also to

$$
\lim _{t \rightarrow \infty} \frac{1}{t} \log \left(\sum_{j=1}^{n} X_{j}(t)\right)=\lim _{t \rightarrow \infty} \frac{1}{t} \log X_{(1)}(t)=\frac{\beta \sigma^{2}}{2 \phi(1)}, \quad \text { a.s. }
$$


for the total capitalization of the entire market and for the biggest stock $X_{(1)}(t)=$ $\max _{1 \leq j \leq n} X_{j}(t)$. In particular, it follows from (4.11), (4.12) that the model of (4.2) with $\alpha>0$ is coherent:

$$
\lim _{t \rightarrow \infty} \frac{1}{t} \log \mu_{i}(t)=0, \quad \text { a.s. }
$$

We also have the following computation for the long-term average of an individual variance:

$$
\lim _{T \rightarrow \infty} \frac{1}{T} \int_{0}^{T} a_{i i}(t) \mathrm{d} t=\lim _{T \rightarrow \infty} \frac{\sigma^{2}}{T} \int_{0}^{T} \frac{1}{\left(\mu_{i}(t)\right)^{2 \beta}} \mathrm{d} t=\frac{\beta \sigma^{4}}{\alpha \phi(1)}, \quad \text { a.s. }
$$

To see this, note from (4.3) that

$$
\begin{aligned}
\int_{0}^{T} \frac{1}{\left(\mu_{i}(t)\right)^{2 \beta}} \mathrm{d} t & =\int_{0}^{T} \frac{\left(\sum_{j=1}^{n} X_{j}(t)\right)^{2 \beta}}{\left(X_{i}(t)\right)^{2 \beta}} \mathrm{d} t=\int_{0}^{T} \frac{A^{\prime}(t)}{(\beta \sigma)^{2} Z_{i}(A(t))} \mathrm{d} t \\
& =\frac{1}{(\beta \sigma)^{2}} \int_{0}^{A(T)} \frac{1}{Z_{i}(\xi)} \mathrm{d} \xi
\end{aligned}
$$

and because $A(\infty)=\infty$ we deduce from (4.4), (4.6):

$$
\begin{aligned}
\frac{1}{T} \int_{0}^{T} \frac{1}{\left(\mu_{i}(t)\right)^{2 \beta}} \mathrm{d} t= & \frac{1 /(\beta \sigma)^{2}}{T} \int_{0}^{A(T)} \frac{1}{Z_{i}(\xi)} \mathrm{d} \xi=\left.\frac{1 / \log \theta \int_{0}^{\theta}\left(Z_{i}(\xi)\right)^{-1} \mathrm{~d} \xi}{(\beta \sigma)^{2} \Upsilon(\theta) / \log \theta}\right|_{\theta=A(T)} \\
& \underset{T / \infty}{\longrightarrow} \frac{1 /(m-2)}{\phi(1)}=\frac{\beta \sigma^{2}}{\alpha \phi(1)}, \quad \text { a.s. }
\end{aligned}
$$

where in the last equality we substituted $m=2+\alpha /\left(\beta \sigma^{2}\right)$. The claim (4.13) follows from this.

\subsection{Proofs of (4.4) and (4.6)}

\subsubsection{Proof of (4.4)}

We shall show that for a squared-Bessel process $Z(\cdot)$ in "dimension" $\mathfrak{d}>2$, namely, a diffusion process that satisfies the SDE

$$
\mathrm{d} Z(t)=\mathfrak{d} \mathrm{d} t+2 \sqrt{Z(t)} \mathrm{d} V(t), \quad Z(0)=z \geq 0
$$


of (2.54) with $V(\cdot)$ a standard Brownian motion, we have

$$
\lim _{t \rightarrow \infty} \frac{1}{\log t} \int_{1}^{t} \frac{1}{Z(s)} \mathrm{d} s=\frac{1}{\mathfrak{d}-2}, \quad \text { a.s. }
$$

With $z>0$ as in (2.54), this will prove the claim (4.4). Notice that any other positive number (i.e. take $c>0$ instead of 1) taken as the lower bound of the integral in (4.15) yields the same limit.

As we mentioned already, this statement was proved in Lemma 4.2 in Cherny (2000). In fact, this result (for an integer dimension $m$ ) is stated as Exercise (3.20), Chapter X in Revuz and Yor (1999). For completeness, we repeat the proof here, as we will use the same approach in proving (4.6).

Suppose first $z=0$; then we have the scaling property

$$
\left(\frac{1}{c} Z(c t)\right)_{t \geq 0} \stackrel{\text { law }}{=}(Z(t))_{t \geq 0} \quad \text { for any } c>0
$$

which follows from the scaling property of Brownian motion (see Revuz and Yor (1999), (1.6) Proposition, p.443). This scaling property implies that the sequence of random variables

$$
\xi_{k} \triangleq \int_{\mathrm{e}^{k}}^{\mathrm{e}^{k+1}} \frac{1}{Z(s)} \mathrm{d} s, \quad k=0,1, \ldots
$$

is stationary; for instance,

$$
\xi_{1}=\int_{\mathrm{e}}^{\mathrm{e}^{2}} \frac{1}{Z(s)} \mathrm{d} s=\int_{1}^{\mathrm{e}} \frac{1}{\frac{1}{\mathrm{e}} Z(\mathrm{e} s)} \mathrm{d} s \stackrel{\text { law }}{=} \int_{1}^{\mathrm{e}} \frac{1}{Z(s)} \mathrm{d} s=\xi_{0},
$$

and so on. Therefore, we can apply Birkhoff's ergodic theorem and obtain

$$
\frac{1}{n} \int_{1}^{\mathrm{e}^{n}} \frac{1}{Z(s)} \mathrm{d} s=\frac{1}{n} \sum_{k=0}^{n-1} \xi_{k} \underset{n / \infty}{\longrightarrow} \mathbb{E}\left(\xi_{0} \mid \mathcal{I}\right), \quad \text { a.s. }
$$

where $\mathcal{I}$, the $\sigma$-algebra of invariant sets, is included in the tail $\sigma$-algebra

$$
\mathcal{T}:=\bigcap_{n \in \mathbb{N}} \sigma\left(\xi_{n}, \xi_{n+1}, \ldots\right)
$$


of the sequence $\left\{\xi_{k}\right\}_{k \in \mathbb{N}_{0}}$. This is, in turn, included in the tail $\sigma$-algebra of the squared-Bessel process, namely $\bigcap_{t>0} \sigma(Z(s), s \geq t)=: \mathcal{Z}$.

We claim that the $\sigma$-algebra $\mathcal{Z}$ is trivial, and argue as follows: thanks to the strong solvability of the stochastic differential equation (4.14) we know that the tail $\sigma$-algebra $\mathcal{Z}$ is the same as the tail $\sigma$-algebra of the Brownian motion $V(\cdot)$, namely $\mathcal{V} \triangleq \bigcap_{t>0} \sigma(V(s), s \geq t)$. Then Blumenthal's zero-one law (see Blumenthal (1957)) implies that the $\sigma$-algebra $\mathcal{F}^{V}(0+)=\bigcap_{t>0} \mathcal{F}^{V}(t)=\bigcap_{t>0} \sigma(V(s), s \leq t)$ is trivial. Furthermore, we have the time-inversion property for the Brownian motion $V(\cdot)$, namely

$$
(t V(1 / t))_{t \geq 0} \stackrel{\text { law }}{=}(V(t))_{t \geq 0}
$$

which means that the triviality of $\mathcal{F}^{V}(0+)$ implies the triviality of $\mathcal{V}$. Consequently, the $\sigma$-algebra $\mathcal{Z}$ is trivial.

But then the $\sigma$-algebra $\mathcal{I}$ is also trivial, so we can compute the expectation in $(4.16)$ as

$$
\mathbb{E}\left(\xi_{0} \mid \mathcal{I}\right)=\mathbb{E}\left(\xi_{0}\right)=\int_{1}^{\mathrm{e}} f(s) \mathrm{d} s=\int_{1}^{\mathrm{e}} \frac{f(1)}{s} \mathrm{~d} s=f(1)=\frac{1}{\mathfrak{d}-2},
$$

where

$$
f(s) \triangleq \mathbb{E}\left(\frac{1}{Z(s)}\right)=\int_{0}^{\infty} \frac{1}{z} \cdot \frac{z^{\mathfrak{d} / 2-1} \mathrm{e}^{-z /(2 s)}}{(2 s)^{\mathfrak{d} / 2} \Gamma(\mathfrak{d} / 2)} \mathrm{d} z=\frac{f(1)}{s}
$$

and

$$
\begin{aligned}
f(1) & =\int_{0}^{\infty} \frac{z^{\mathfrak{d} / 2-2} \mathrm{e}^{-z / 2}}{2^{\mathfrak{d} / 2} \Gamma(\mathfrak{d} / 2)} \mathrm{d} z=\int_{0}^{\infty} \frac{z^{\ell / 2-1} \mathrm{e}^{-z / 2}}{2^{1+\ell / 2} \Gamma(1+\ell / 2)} \mathrm{d} z \\
& =\int_{0}^{\infty} \frac{2^{\ell / 2-1} u^{\ell / 2-1} \mathrm{e}^{-u}}{2^{1+\ell / 2} \Gamma(1+\ell / 2)} 2 \mathrm{~d} u=\frac{1}{2 \Gamma(1+\ell / 2)} \int_{0}^{\infty} u^{\ell / 2-1} \mathrm{e}^{-u} \mathrm{~d} u \\
& =\frac{\Gamma(\ell / 2)}{2 \Gamma(1+\ell / 2)}=\frac{1}{\ell}
\end{aligned}
$$

for $\ell \triangleq \mathfrak{d}-2$. Note that we have used the known formula for the transition density of the $\mathfrak{d}$-dimensional squared-Bessel process (see for instance, Revuz and Yor (1999), p.441, Corollary 1.4) and $\Gamma(\cdot)$ denotes the Gamma function. 
Since the process $Z(\cdot)$ is positive, (4.16) and (4.17) together imply

$$
\lim _{t \rightarrow \infty} \frac{1}{\log t} \int_{1}^{t} \frac{1}{Z(s)} \mathrm{d} s=\lim _{n \rightarrow \infty} \frac{1}{n} \int_{1}^{\mathrm{e}^{n}} \frac{1}{Z(s)} \mathrm{d} s=\mathbb{E}\left(\xi_{0} \mid \mathcal{I}\right)=\frac{1}{\mathfrak{d}-2}, \quad \text { a.s. }
$$

Now for any given $z>0$, the first hitting time $T_{z} \triangleq \inf \left\{t \geq 0: Z_{t}=z\right\}$ is a.s. finite (since any squared-Bessel process in "dimension" $\mathfrak{d}>2$ is transient); and by the strong Markov property, $\hat{Z}(\cdot)=Z\left(\cdot+T_{Z}\right)$ is also a squared-Bessel process, but started at $z$. From what we have already established we deduce

$$
\frac{1}{\log t} \int_{0}^{t} \frac{1}{\hat{Z}(s)} \mathrm{d} s=\frac{1}{\log t} \int_{T_{z}}^{t+T_{z}} \frac{1}{Z(s)} \mathrm{d} s \underset{t / \infty}{\underset{\mathfrak{d}}{\longrightarrow}} \frac{1}{\mathrm{~d}}, \quad \text { a.s. }
$$

and the proof of (4.15), thus also (4.4), is complete.

\subsubsection{Proof of (4.6)}

We shall show that for $n$ independent copies $Z_{1}(\cdot), \ldots, Z_{n}(\cdot)$ of the squared-Bessel process in "dimension" $m \geq 2$, namely

$$
\mathrm{d} Z_{j}(t)=m \mathrm{~d} t+2 \sqrt{Z_{j}(t)} \mathrm{d} V_{j}(t), \quad Z_{j}(0)=z_{j} \geq 0
$$

and $V_{1}(\cdot), \ldots, V_{n}(\cdot)$ independent Brownian motions, we have

$$
\lim _{t \rightarrow \infty} \frac{1}{\log t} \int_{1}^{t} \frac{1}{\left(\sum_{j=1}^{n}\left(Z_{j}(s)\right)^{1 /(2 \beta)}\right)^{2 \beta}} \mathrm{d} s=\phi(1) \in(0, \infty), \quad \text { a.s. }
$$

in the notation of (4.7). With $z_{1}>0, \ldots, z_{n}>0$ this will prove claim (4.6). Notice that any other positive number (i.e. take $c>0$ instead of 1 ) taken as the lower bound of the integral in (4.20) yields the same limit.

From the definition of $\phi(\theta)$ in (4.7) it is obvious that $\phi(\theta)=\phi(1) / \theta>0$ for $0<\theta<\infty$. To show that $\phi(1)<\infty$, consider first the case when $m>2$. Note that, if we introduce independent random variables $Q_{1}, \ldots, Q_{n}$ with common distribution

$$
\mathbb{P}\left(Q_{j} \leq q\right)=\int_{0}^{q} \frac{z^{m / 2-1} \mathrm{e}^{-z / 2}}{2^{m / 2} \Gamma(m / 2)} \mathrm{d} z
$$


for $0 \leq q<\infty$, then the limiting quantity (4.20) can be expressed as

$$
\phi(1)=\mathbb{E}\left(\sum_{j=1}^{n} Q_{j}^{1 /(2 \beta)}\right)^{-2 \beta} \leq \mathbb{E}\left(\frac{1}{Q_{1}}\right)=f(1)=\frac{1}{m-2},
$$

where the inequality follows from the fact that the variables $Q_{j}$ are non-negative for all $j=1, \ldots, n$. We have recalled here the notation and computation of (4.18).

In the case $m=2$ (which corresponds to $\alpha=0$ ), we have

$$
\begin{aligned}
\phi(1) & =\int_{0}^{\infty} \cdots \int_{0}^{\infty} 2^{-n}\left(\sum_{j=1}^{n}\left(q_{j}\right)^{1 /(2 \beta)}\right)^{-2 \beta} \mathrm{e}^{-\left(q_{1}+\cdots+q_{n}\right) / 2} \mathrm{~d} q_{1} \ldots \mathrm{d} q_{n} \\
& =\mathbb{E}\left(\sum_{j=1}^{n} Q_{j}^{1 /(2 \beta)}\right)^{-2 \beta},
\end{aligned}
$$

where $Q_{1}, \ldots, Q_{n}$ are independent random variables with common exponential $\lambda=$ $1 / 2$ distribution (equivalently, chi-square with two degrees of freedom). But then

$$
\phi(1) \leq \mathbb{E}\left(\max _{1 \leq j \leq n} Q_{j}\right)^{-1},
$$

together with

$$
\mathbb{P}\left(\max _{1 \leq j \leq n} Q_{j} \leq q\right)=\left(1-\mathrm{e}^{-q / 2}\right)^{n}
$$

and

$$
\frac{\mathrm{d}}{\mathrm{d} q} \mathbb{P}\left(\max _{1 \leq j \leq n} Q_{j} \leq q\right)=\frac{n}{2} \mathrm{e}^{-q / 2}\left(1-\mathrm{e}^{-q / 2}\right)^{n-1},
$$

implies that for $n \geq 2$ we have

$$
\begin{aligned}
\phi(1) & \leq \int_{0}^{\infty} \frac{n}{2 q} \mathrm{e}^{-q / 2}\left(1-\mathrm{e}^{-q / 2}\right)^{n-1} \mathrm{~d} q \leq \int_{0}^{\infty} \frac{n}{2 q} \mathrm{e}^{-q / 2}\left(\frac{q}{2}\right)^{n-1} \mathrm{~d} q \\
& =\frac{n}{2^{n}} \int_{0}^{\infty} \mathrm{e}^{-q / 2} q^{n-2} \mathrm{~d} q<\infty .
\end{aligned}
$$

Hence, we have shown that $\phi(1)$ is positive and finite for any $m \geq 2$.

Now, in order to prove the limiting property in (4.20), we shall mimic the argument that led to the proof of (4.4), starting with the case $z_{1}=\ldots=z_{n}=$ 
0 . Then the independence and scaling properties of the squared-Bessel processes $Z_{1}(\cdot), \ldots, Z_{n}(\cdot)$ show that the random variables

$$
\eta_{i} \triangleq \int_{\mathrm{e}^{i}}^{\mathrm{e}^{i+1}} \frac{1}{\left(\sum_{j=1}^{n}\left(Z_{j}(s)\right)^{1 /(2 \beta)}\right)^{2 \beta}} \mathrm{d} s, \quad i=0,1, \ldots
$$

form a stationary sequence; for instance,

$$
\begin{aligned}
\eta_{1} & =\int_{\mathrm{e}}^{\mathrm{e}^{2}} \frac{1}{\left(\sum_{j=1}^{n}\left(Z_{j}(s)\right)^{1 /(2 \beta)}\right)^{2 \beta}} \mathrm{d} s=\int_{1}^{\mathrm{e}} \frac{1}{\frac{1}{\mathrm{e}}\left(\sum_{j=1}^{n}\left(Z_{j}(\mathrm{e} s)\right)^{1 /(2 \beta)}\right)^{2 \beta}} \mathrm{d} s \\
& =\int_{1}^{\mathrm{e}} \frac{1}{\left(\sum_{j=1}^{n}\left(\frac{1}{\mathrm{e}} Z_{j}(\mathrm{e} s)\right)^{1 /(2 \beta)}\right)^{2 \beta}} \mathrm{d} s \stackrel{\text { law }}{=} \int_{1}^{\mathrm{e}} \frac{1}{\left(\sum_{j=1}^{n}\left(Z_{j}(s)\right)^{1 /(2 \beta)}\right)^{2 \beta}} \mathrm{d} s=\eta_{0},
\end{aligned}
$$

and so on. For this sequence the Birkhoff's ergodic theorem shows

$$
\frac{1}{k} \int_{1}^{e^{k}} \frac{1}{\tilde{Z}(s)} \mathrm{d} s=\frac{1}{k} \sum_{i=0}^{k-1} \eta_{i} \underset{k \rightarrow \infty}{\longrightarrow} \mathbb{E}\left(\eta_{0} \mid \mathcal{I}\right), \quad \text { a.s. }
$$

Here we have denoted

$$
\tilde{Z}(s) \triangleq\left(\sum_{j=1}^{n}\left(Z_{j}(s)\right)^{1 /(2 \beta)}\right)^{2 \beta}, \quad 0 \leq s<\infty,
$$

and $\mathcal{I}$, the $\sigma$-algebra of invariant sets, is included in the tail $\sigma$-algebra of the sequence $\left\{\eta_{k}\right\}_{k \in \mathbb{N}_{0}}$, which is in turn included in the tail $\sigma$-algebra of the $n$-dimensional Brownian motion $V(\cdot)=\left(V_{1}(\cdot), \ldots, V_{n}(\cdot)\right)$ (again by the strong solvability of the stochastic differential equation (4.14)). For this Brownian motion $V(\cdot)$ the $\sigma$-algebra $\mathcal{F}^{V}(0+)=\bigcap_{t>0} \mathcal{F}^{V}(t)$ is trivial, by Blumenthal's zero-one law (see Blumenthal (1957)); and the time-inversion property

$$
(t V(1 / t))_{t \geq 0} \stackrel{\text { law }}{=}(V(t))_{t \geq 0}
$$

shows that $\mathcal{V}=\bigcap_{t>0} \sigma(V(s), s \geq t)$ is trivial. Consequently, $\mathcal{I}$ is trivial as well, and therefore we have

$$
\mathbb{E}\left(\eta_{0} \mid \mathcal{I}\right)=\mathbb{E}\left(\eta_{0}\right)=\int_{1}^{\mathrm{e}} \mathbb{E}\left(\frac{1}{\tilde{Z}(s)}\right) \mathrm{d} s=\int_{1}^{\mathrm{e}} \phi(s) \mathrm{d} s=\int_{1}^{\mathrm{e}} \frac{\phi(1)}{s} \mathrm{~d} s=\phi(1), \quad \text { a.s. }
$$


This together with (4.22) and the fact that the process $\tilde{Z}(\cdot)$ is positive give us

$$
\lim _{t \rightarrow \infty} \frac{1}{\log t} \int_{1}^{t} \frac{1}{\tilde{Z}(s)} \mathrm{d} s=\lim _{k \rightarrow \infty} \frac{1}{k} \int_{1}^{e^{k}} \frac{1}{\tilde{Z}(s)} \mathrm{d} s=\mathbb{E}\left(\eta_{0} \mid \mathcal{I}\right)=\phi(1), \quad \text { a.s. }
$$

We have shown the limiting property in (4.20) for the case $z_{1}=\ldots=z_{n}=0$ (this was important when applying the scaling property). Now we will argue that it holds even for a starting point in the positive orthant, that is, the case when $z_{1}>0, \ldots, z_{n}>0$.

Let us now consider two systems of squared-Bessel processes. Together with the system of $n$ independent squared-Bessel processes $Z_{1}(\cdot), \ldots, Z_{n}(\cdot)$ with starting points $Z_{1}(0)=z_{1}>0, \ldots, Z_{n}(0)=z_{n}>0$ of $(4.19)$, let us denote by $Z_{1}^{0}(\cdot), \ldots, Z_{n}^{0}(\cdot)$ the squared-Bessel processes with the same "dimension" $m$ and the same generating independent Brownian motions $V_{1}(\cdot), \ldots, V_{n}(\cdot)$ as in (4.19) but starting from the origin $Z_{1}^{0}(0)=0, \ldots, Z_{n}^{0}(0)=0$, namely for $j=1, \ldots, n$ we have

$$
\begin{array}{ll}
\mathrm{d} Z_{j}(t)=m \mathrm{~d} t+2 \sqrt{Z_{j}(t)} \mathrm{d} V_{j}(t), & Z_{j}(0)=z_{j}>0, \\
\mathrm{~d} Z_{j}^{0}(t)=m \mathrm{~d} t+2 \sqrt{Z_{j}^{0}(t)} \mathrm{d} V_{j}(t), & Z_{j}^{0}(0)=0,
\end{array}
$$

where $V_{1}(\cdot), \ldots, V_{n}(\cdot)$ are independent Brownian motions. Then the comparison theorem (see Karatzas and Shreve (1991), Proposition 2.18 on p. 293) gives us for every $j=1, \ldots, n$

$$
\mathbb{P}\left(Z_{j}(t) \geq Z_{j}^{0}(t), \forall 0 \leq t<\infty\right)=1 .
$$

Thus, we have

$$
\limsup _{t \rightarrow \infty} \frac{1}{\log t} \int_{1}^{t} \frac{1}{\left(\sum_{j=1}^{n}\left(Z_{j}(s)\right)^{1 /(2 \beta)}\right)^{2 \beta}} \mathrm{d} s
$$




$$
\leq \limsup _{t \rightarrow \infty} \frac{1}{\log t} \int_{1}^{t} \frac{1}{\left(\sum_{j=1}^{n}\left(Z_{j}^{0}(s)\right)^{1 /(2 \beta)}\right)^{2 \beta}} \mathrm{d} s=\phi(1),
$$

thanks to the first part of the proof.

Let us now consider another system of squared-Bessel processes. In particular, let us first denote by $\tilde{Z}_{1}^{0}(\cdot), \ldots, \tilde{Z}_{n}^{0}(\cdot)$ the squared-Bessel processes with the "dimension" $m$ and starting from the origin, that is, for $j=1, \ldots, n$ we have

$$
\mathrm{d} \tilde{Z}_{j}^{0}(t)=m \mathrm{~d} t+2 \sqrt{\tilde{Z}_{j}^{0}(t)} \mathrm{d} W_{j}(t), \quad \tilde{Z}_{j}^{0}(0)=0,
$$

where $W_{1}(\cdot), \ldots, W_{n}(\cdot)$ are independent Brownian motions which are independent of $V_{1}(\cdot), \ldots, V_{n}(\cdot)$. Next we denote $z_{\max }:=\max \left\{z_{1}, \ldots, z_{n}\right\}$ and define a stopping time

$$
\tau:=\inf \left\{t>0: \min _{i=1, \ldots, n} \tilde{Z}_{i}^{0}(t)=z_{\max }\right\} .
$$

we know that $\tau<\infty$ a.s., since the squared-Bessel processes in "dimension" greater than two are transient, and the squared-Bessel processes $Z$ in dimension two satisfies $\mathbb{P}\left(\sup _{t} Z(t)=\infty\right)=1$. After time $\tau$, we use the processes $V_{1}(\cdot)+W_{1}(\tau), \ldots, V_{n}(\cdot)+$ $W_{n}(\tau)$ as the generating Brownian motions for the processes $\tilde{Z}_{1}^{0}(\cdot), \ldots, \tilde{Z}_{n}^{0}(\cdot)$ and we also define for $j=1, \ldots, n$ the processes

$$
\tilde{Z}_{j}(t):=\tilde{Z}_{j}^{0}(\tau+t), \quad 0 \leq t<\infty
$$

Therefore, we have

$$
\begin{array}{ll}
\mathrm{d} Z_{j}(t)=m \mathrm{~d} t+2 \sqrt{Z_{j}(t)} \mathrm{d} V_{j}(t), & Z_{j}(0)=z_{j} \leq z_{\max }, \\
\mathrm{d} \tilde{Z}_{j}(t)=m \mathrm{~d} t+2 \sqrt{\tilde{Z}_{j}(t)} \mathrm{d} V_{j}(t), & \tilde{Z}_{j}(0) \geq z_{\max }
\end{array}
$$

Using the comparison theorem again, we obtain for every $j=1, \ldots, n$

$$
\mathbb{P}\left(Z_{j}(t) \leq \tilde{Z}_{j}(t), \forall 0 \leq t<\infty\right)=1
$$


Thus, we have

$$
\begin{aligned}
& \liminf _{t \rightarrow \infty} \frac{1}{\log t} \int_{1}^{t} \frac{1}{\left(\sum_{j=1}^{n}\left(Z_{j}(s)\right)^{1 /(2 \beta)}\right)^{2 \beta}} \mathrm{d} s \\
& \quad \geq \liminf _{t \rightarrow \infty} \frac{1}{\log t} \int_{1}^{t} \frac{1}{\left(\sum_{j=1}^{n}\left(\tilde{Z}_{j}(s)\right)^{1 /(2 \beta)}\right)^{2 \beta}} \mathrm{d} s \\
& \quad=\liminf _{t \rightarrow \infty} \frac{1}{\log t} \int_{\tau}^{t} \frac{1}{\left(\sum_{j=1}^{n}\left(\tilde{Z}_{j}^{0}(s)\right)^{1 /(2 \beta)}\right)^{2 \beta}} \mathrm{d} s=\phi(1),
\end{aligned}
$$

a.s.

thanks to the first part of the proof.

Combining (4.25) and (4.29) together, we see that the limiting property in (4.20) holds also in the case when $z_{1}>0, \ldots, z_{n}>0$.

This finishes the proof of (4.6). 


\section{Chapter 5}

\section{Conclusion and future directions}

As a conclusion, we will provide in this Chapter a brief list of related open questions, possible extensions and future research directions.

\section{$5.1 \quad$ Study of explosions}

Let us return to the discussion in Section 2.3, where we stated sufficient conditions on the given function $K(\cdot)$, so that the properties (P1) and (P2), as in (2.62) and (2.63), are satisfied by the time-change process $\Upsilon(\cdot)$ defined in $(2.61)$, with $\mathcal{T}(\cdot)$ defined in (2.3). As we already noted, there exists a weak solution of the system in (2.1) even if these properties are not satisfied, although this solution may then explode in finite time. We will state here sufficient conditions under which these properties are not satisfied.

First, we state sufficient conditions on the function $K(\cdot)$ which imply that the time-change process $\Upsilon(\cdot)$ defined in (2.61), fails to satisfy property (P1) in (2.62), namely, the process $\Upsilon(\cdot)$ a.s. explodes in finite time. There still exists a weak solution of the system in (2.1) that is unique in distribution, for more details see Pickova (2013). 
Proposition 5.1. Assume $K(\cdot):(0, \infty)^{n} \rightarrow(0, \infty)$ is a measurable function and there exists a measurable function $f:(0, \infty) \rightarrow(0, \infty)$ such that for all $x=\left(x_{1}, \ldots, x_{n}\right) \in(0, \infty)^{n}$

$$
K(x) \leq f\left(\|x\|_{2 \beta}\right), \quad \forall x=\left(x_{1}, \ldots, x_{n}\right) \in(0, \infty)^{n},
$$

where $\|x\|_{p}:=\left(x_{1}^{p}+\cdots+x_{n}^{p}\right)^{1 / p}$ for any $p>0$, and the function $u \mapsto\left[u f\left(u^{1 / \beta}\right)\right]^{-2}$ fails to be locally integrable on $(0, \infty)$. Then the time-change process $\Upsilon(\cdot)$ defined in (2.61) fails to satisfy property (P1) in (2.62).

Proof. The proof is similar to the proof of Proposition 2.3 in Section 2.3.2. First, we recall the expression for the process $\Upsilon(\cdot)$ in $(2.73)$, namely

$$
\Upsilon(\theta)=C_{\beta, \sigma, n} \int_{0}^{\theta}\left[K\left(\left(Z_{1}(\xi)\right)^{\frac{1}{2 \beta}}, \ldots,\left(Z_{n}(\xi)\right)^{\frac{1}{2 \beta}}\right)\right]^{-2}\left[\sum_{j=1}^{n}\left(Z_{j}(\xi)\right)^{\frac{1}{2 \beta}}\right]^{-2 \beta} \mathrm{d} \xi
$$

where $C_{\beta, \sigma, n}$ is a scaling constant depending only on $\beta, \sigma$, and $n$. Assuming (5.1), we obtain from (5.2) and from the inequalities in (2.75), the following bound

$$
\begin{aligned}
\Upsilon(\theta) / C_{\beta, \sigma, n} & \geq \int_{0}^{\theta}\left[f\left(\left\|\left(\left(Z_{1}(\xi)\right)^{\frac{1}{2 \beta}}, \ldots,\left(Z_{n}(\xi)\right)^{\frac{1}{2 \beta}}\right)\right\|_{2 \beta}\right)\right]^{-2}\left[\sum_{j=1}^{n}\left(Z_{j}(\xi)\right)^{\frac{1}{2 \beta}}\right]^{-2 \beta} \mathrm{d} \xi \\
& \geq n^{-2 \beta} \int_{0}^{\theta}\left[f\left(\|\left(Z_{1}(\xi), \ldots,\left(Z_{n}(\xi)\right) \|_{1}^{\frac{1}{2 \beta}}\right)\right]^{-2}\left(\sum_{j=1}^{n} Z_{j}(\xi)\right)^{-1} \mathrm{~d} \xi\right.
\end{aligned}
$$

Therefore

$$
\Upsilon(\theta) / C_{\beta, \sigma, n} \geq n^{-2 \beta} \int_{0}^{\theta}\left[R(\xi) f\left((R(\xi))^{\frac{1}{\beta}}\right)\right]^{-2} \mathrm{~d} \xi
$$

where we have noted that $R(\cdot):=\sqrt{Z(\cdot)}=\sqrt{Z_{1}(\cdot)+\ldots+Z_{n}(\cdot)}$ is a Bessel process in dimension $m=m_{1}+\ldots+m_{n}>2$. The claim follows from Proposition A.1, and from the assumption that the function $u \mapsto\left[u f\left(u^{1 / \beta}\right)\right]^{-2}$ fails to be locally integrable on $(0, \infty)$.

Let us now state sufficient conditions on the function $K(\cdot)$ which imply that the time-change process $\Upsilon(\cdot)$ defined in (2.61), fails to satisfy property (P2) in 
(2.63), that is $\Upsilon(\theta) \nrightarrow \infty$ a.s. as $\theta \rightarrow \infty$, thus the process $A(\cdot)$ explodes in finite time (recall, that it is defined as the inverse of the process $\Upsilon(\cdot)$ ). As a consequence, under these conditions, explosion of the system in (2.1) does occur in finite time.

We consider, as before, a measurable function $K(\cdot):(0, \infty)^{n} \rightarrow(0, \infty)$ and the following conditions:

1. There exists an integer $\nu \in\{1, \ldots, n\}$ and a measurable function $f:(0, \infty) \rightarrow$ $(0, \infty)$ such that

$$
K(x) \geq f\left(x_{\nu}\right), \quad \forall x=\left(x_{1}, \ldots, x_{n}\right) \in(0, \infty)^{n},
$$

the function $u \mapsto\left[u f\left(u^{1 / \beta}\right)\right]^{-2}$ is locally integrable on $\left(0, a_{\nu}\right]$, and

$$
\int_{a_{\nu}}^{\infty}\left[u f^{2}\left(u^{1 / \beta}\right)\right]^{-1} \mathrm{~d} u<\infty,
$$

where $a_{\nu}:=Z_{\nu}(0)=1 /(\beta \sigma)^{2} x_{\nu}^{2 \beta}>0$;

2. There exists a non-increasing measurable function $f:(0, \infty) \rightarrow(0, \infty)$ such that

$$
K(x) \geq f\left(\|x\|_{2 \beta}\right), \quad \forall x=\left(x_{1}, \ldots, x_{n}\right) \in(0, \infty)^{n},
$$

the function $u \mapsto\left[u f\left(u^{1 / \beta}\right)\right]^{-2}$ is locally integrable on $(0, a]$, and

$$
\int_{a}^{\infty}\left[u f^{2}\left(u^{1 / \beta}\right)\right]^{-1} \mathrm{~d} u<\infty,
$$

where $a:=\sum_{i=1}^{n} Z_{i}(0)=1 /(\beta \sigma)^{2} \sum_{i=1}^{n} x_{i}^{2 \beta}>0$.

Proposition 5.2. Assume $K(\cdot):(0, \infty)^{n} \rightarrow(0, \infty)$ is a measurable function, and one of the above conditions holds. Then the time-change process $\Upsilon(\cdot)$ defined in (2.61) fails to satisfy property (P2) in (2.63), which means that its inverse, the process $A(\cdot)$, explodes in finite time. 
Proof. The proof is similar to the proof of Proposition 2.2 in Section 2.3.1. First, recalling the expression (5.2) and assuming (5.3), we obtain

$$
\begin{aligned}
\Upsilon(\theta) / C_{\beta, \sigma, n} & \leq \int_{0}^{\theta}\left[f\left(\left(Z_{\nu}(\xi)\right)^{\frac{1}{2 \beta}}\right)\right]^{-2}\left(Z_{\nu}(\xi)\right)^{-1} \mathrm{~d} \xi \\
& =\int_{0}^{\theta}\left[R_{\nu}(\xi) f\left(\left(R_{\nu}(\xi)\right)^{\frac{1}{\beta}}\right)\right]^{-2} \mathrm{~d} \xi
\end{aligned}
$$

where $R_{\nu}(\cdot):=\sqrt{Z_{\nu}(\cdot)}$ denotes a Bessel process in dimension $m_{\nu} \geq 2$. If $m_{\nu}>2$, then the claim follows from Proposition A.2, as we assume (5.4) and the function $\left[u f\left(u^{1 / \beta}\right)\right]^{-2}$ is locally integrable on $\left(0, a_{\nu}\right]$. If $m_{\nu}=2$, then the claim follows Proposition A.3.

In the second case, using (5.5) and noting that $f(\cdot)$ is assumed to be a nonincreasing function, we obtain

$$
\begin{aligned}
\Upsilon(\theta) / C_{\beta, \sigma, n} & \leq \int_{0}^{\theta}\left[f\left\|\left(\left(Z_{1}(\xi)\right)^{\frac{1}{2 \beta}}, \ldots,\left(Z_{n}(\xi)\right)^{\frac{1}{2 \beta}}\right)\right\|_{2 \beta}\right]^{-2}\left[\sum_{j=1}^{n}\left(Z_{j}(\xi)\right)^{\frac{1}{2 \beta}}\right]^{-2 \beta} \mathrm{d} \xi \\
& \leq \int_{0}^{\theta}\left[f \|\left(Z_{1}(\xi), \ldots,\left(Z_{n}(\xi)\right) \|_{1}^{\frac{1}{2 \beta}}\right]^{-2}\left(Z_{1}(\xi)\right)^{-1} \mathrm{~d} \xi\right. \\
& \leq \int_{0}^{\theta}\left[f\left(\left(Z_{1}(\xi)\right)^{\frac{1}{2 \beta}}\right)\right]^{-2}\left(Z_{1}(\xi)\right)^{-1} \mathrm{~d} \xi
\end{aligned}
$$

Hence, if we denote $R_{1}(\cdot):=\sqrt{Z_{1}(\cdot)}$, a Bessel process in dimension $m_{1} \geq 2$, we have the following bound

$$
\Upsilon(\theta) / C_{\beta, \sigma, n} \leq \int_{0}^{\theta}\left[R_{1}(\xi) f\left(\left(R_{1}(\xi)\right)^{\frac{1}{\beta}}\right)\right]^{-2} \mathrm{~d} \xi .
$$

Now again, if $m_{\nu}>2$, then the claim follows from Proposition A.2, as we assume (5.6) and the function $\left[u f\left(u^{1 / \beta}\right)\right]^{-2}$ is locally integrable on $(0, a]$. If $m_{\nu}=2$, then the claim follows Proposition A.3.

One might be interested to know more about the system in (2.1) in the case when explosions occur, for instance to know what the distribution of the time-toexplosion is. Recently, Karatzas and Ruf (2013) examine the distribution of the 
time-to-explosion for linear diffusions. To the best of our knowledge, no similar work has been done in the multi-dimensional setting, and remains to be explored in the future.

In the case of the models presented in this thesis, we could see that the timeto-explosion can be expressed as an integral functional of squared-Bessel processes, namely

$$
\int_{0}^{\infty}\left[\mathcal{T}\left((\beta \sigma)^{\frac{1}{\beta}}\left(Z_{1}(\xi)\right)^{\frac{1}{2 \beta}}, \ldots,(\beta \sigma)^{\frac{1}{\beta}}\left(Z_{n}(\xi)\right)^{\frac{1}{2 \beta}}\right)\right]^{-2} \mathrm{~d} \xi
$$

where $\mathcal{T}(\cdot)$ is defined in $(2.3)$, and the processes $Z_{1}(\cdot), \ldots, Z_{n}(\cdot)$ are squared-Bessel processes as in (2.60).

\subsection{Invariant distribution of the market weights}

In Section 3.3 we derived the dynamics for the market weights if the market model is determined by the system in (2.1). For the case of volatility-stabilized markets, that is the case of $\beta=1 / 2$ and $K(\cdot) \equiv 1$, Pal (2011) studies the market weights in more detail, in particular, it is shown that the law of the market weights is the same as that of a multi-allele Wright-Fisher model with mutation parameters given by the parameters $\alpha_{1}, \ldots, \alpha_{n}$. Moreover, the joint density of market weights at fixed times and certain stopping times in volatility-stabilized models is also determined.

One may be interested to know for what choices of the parameters the invariant distribution of the market weights exists in the more general setting of (2.1) presented in this thesis, respectively, one may wish to determine how the invariant distribution looks like. It is not possible to simply use the same approach as in Pal (2011), where the results utilize the fact that the time change process in the case of volatility-stabilized models is an integral functional of the total capitalization process, and this process is shown to be be independent from the vector-valued process of the market weights. However, this is not true in the more general setting, hence 
one would need to find a new approach.

\subsection{Other possible extensions}

More results have been derived for the case of volatility-stabilized models, and could possibly be extended to the more general setting of models presented here. For instance, Shkolnikov (2013) investigates the behavior of volatility-stabilized market models when the number of stocks $n$ approaches infinity. It would also be of interest to know how the market properties depend on the choice of parameter $\beta$. Some dependence on $\beta$ was discussed in Section 3.2 where we show that the value of $\beta$ affects the time one needs to wait for some arbitrage opportunities.

Also, it shall be possible to say more about the behavior of the model as time approaches infinity, in particular, to derive the large deviation principle, at least in the case when $K(\cdot) \equiv 1$, using the already known results for Bessel-square processes.

Another open problem is whether it is possible to extend the models studied in this thesis to the case when the driving Brownian motions $W_{1}(\cdot), \ldots, W_{n}(\cdot)$ are correlated, in other words, it is not known whether there exists a weak solution to the system in (2.1) in this case. 


\section{Bibliography}

Banner, A. and Fernholz, D. (2008). Short-term relative arbitrage in volatilitystabilized markets. Annals of Finance, 4:445-454.

Bass, R. F. and Perkins, E. A. (2002). Degenerate stochastic differential equations with Hölder continuous coefficients and super-Markov chains. Transactions of the American Mathematical Society, 355:373-405.

Blumenthal, R. M. (1957). An extended Markov property. Transactions of the American Mathematical Society, 85:52-72.

Cherny, A. S. (2000). Convergence of some integrals associated with Bessel processes. Teor. Veroyatnost. i Primenen., 45:251-267.

Dubins, L. and Schwarz, G. (1967). On extremal martingale distributions. Proc. 5th Berkeley Symp. Math. Stat. Prob., Univ. California II, part I, pages 295-299.

Engelbert, H. J. and Schmidt, W. (1987). On the behaviour of certain Bessel functionals. An application to a class of stochastic differential equations. Math. Nachr., $131: 219-234$.

Fernholz, E. R. (2002). Stochastic Portfolio Theory. Springer, New York.

Fernholz, E. R. and Karatzas, I. (2005). Relative arbitrage in volatility-stabilized markets. Annals of Finance, 1(2):149-177.

Fernholz, E. R. and Karatzas, I. (2009). Stochastic Portfolio Theory: An Overview. Mathematical Modelling and Numerical Methods in Finance, Bensoussan, A., and Zhang, Q. (Guest Editors), Special Volume of Handbook of Numerical Analysis, Ciarlet, P.G. (Editor), Volume XV:89-167. Elsevier.

Goia, I. (2009). Bessel and volatility-stabilized processes. ProQuest LLC, Ann Arbor, MI. Thesis (Ph.D.)-Columbia University.

Karatzas, I. and Ruf, J. (2013). Distribution of the time to explosion for onedimensional diffusions. Preprint. 
Karatzas, I. and Shreve, S. E. (1991). Brownian Motion and Stochastic Calculus. Springer, New York, Second edition.

Krylov, N. (1969). On Itô's stochastic differential equations. Theory of Probability and Its Applications, 14(2):330-336.

Pal, S. (2011). Analysis of market weights under volatility-stabilized market models. Annals of Applied Probability, 21(3):1180-1213.

Pickova, R. (2013). Generalized volatility-stabilized processes. Annals of Finance. To appear, DOI: 10.1007/s10436-013-0230-9.

Revuz, D. and Yor, M. (1999). Continuous Martingales and Brownian Motion. Springer, Berlin, Third edition.

Rogers, L. and Williams, D. (2000). Diffusions, Markov Processes and Martingales, volume 2. Cambridge University Press, Cambridge, Second edition.

Shkolnikov, M. (2013). Large volatility-stabilized markets. Stochastic Processes and Their Applications, 123:212-228.

Xue, X.-X. (1990). A zero-one law for integral functionals of the Bessel process. Séminaire de Probabilités (Strassbourg), 24:137-153.

Yamada, T. and Watanabe, S. (1971). On the uniqueness of solutions of stochastic differential equations. J. Math. Kyoto Univ., 11:155-167. 


\section{Appendix A}

\section{Bessel and Squared-Bessel}

\section{Processes}

\section{A.1 Definition and Basic Properties}

A Bessel process with dimension $d$, where $d \geq 2$ is an integer, is defined as a process $R(\cdot)$ that satisfies the stochastic differential equation

$$
\mathrm{d} R(u)=\frac{d-1}{2 R(u)} \mathrm{d} u+\mathrm{d} B(u), R(0)=r>0, \quad 0 \leq u<\infty
$$

where $B(\cdot)$ is a Brownian motion. It can be checked easily that the squared-Bessel process $Z(\cdot)=(R(\cdot))^{2}$ satisfies the following stochastic differential equation

$$
\mathrm{d} Z(u)=d \mathrm{~d} u+2 \sqrt{Z(u)} \mathrm{d} B(u), Z(0)=z>0, \quad 0 \leq u<\infty .
$$

According to results of Yamada-Watanabe (see, for instance Proposition 2.13 on p.291 in Karatzas and Shreve (1991)), each of these equations admits a pathwise unique, strong solution with the values in $(0, \infty)$. Bessel processes can also be defined as the modulus (resp., distance from the origin) of the $d$-dimensional Brownian motion (see Definition 3.19 on p.158 in Karatzas and Shreve (1991)). 
In fact both stochastic differential equation, (A.1) and (A.2), admit a pathwise unique, strong and strictly positive solution even for non-integer values of “dimension" parameter $d$ (see, for instance Revuz and Yor (1999), p. 439).

We will state here known results regarding convergence and divergence of integral functionals of Bessel processes that we use in the proofs of the results in Section 2.3 and in Section 5.1. When examining finite as well as infinite integral functionals of Bessel processes it is necessary to treat different cases separately, since the sufficient and necessary conditions differ depending not only on the dimension of the process, but also on whether the process starts from the origin or from a different (positive) initial value. For a detailed discussion of different cases, we refer the reader to Engelbert and Schmidt (1987) and to Xue (1990).

The first proposition below treats the case of finite integral functionals, and the following proposition deals with the case of infinite integral functionals.

Proposition A.1. For a Bessel process $R(\cdot)$ with dimension $d \geq 2$ and $R(0)=$ $r>0$, and for a measurable function $h:(0, \infty) \mapsto[0, \infty)$, consider the following statements

1. $\mathbb{P}\left(\int_{0}^{t} h\left(R_{s}\right) \mathrm{d} s<\infty, \forall 0 \leq t<\infty\right)>0 ;$

2. $\mathbb{P}\left(\int_{0}^{t} h\left(R_{s}\right) \mathrm{d} s<\infty, \forall 0 \leq t<\infty\right)=1$;

3. $f h$ is locally integrable on $(0, \infty)$.

For $d=2$, all three statements are equivalent. For $d>2$ the statements 2. and 3. are equivalent, however the so-called zero-one law (i.e. equivalence of 1. and 2.) does not hold when $d>2$.

Proof. See Theorem 1 in Engelbert and Schmidt (1987), and Remark 5 in Xue (1990). 
Proposition A.2. Let $R(\cdot)$ be a Bessel process with dimension $d>2$ and $R(0)=$ $r>0$. If a measurable function $f h:(0, \infty) \mapsto[0, \infty)$ satisfies

$$
\int_{r}^{\infty} h(x) x \mathrm{~d} x=\infty
$$

then

$$
\mathbb{P}\left(\int_{0}^{\infty} h\left(R_{s}\right) \mathrm{d} s=\infty\right)=1 .
$$

If additionally we assume that $f$ is locally integrable on $(0, r]$, then we have the $0-1$ law, that is

$$
\mathbb{P}\left(\int_{0}^{\infty} h\left(R_{s}\right) \mathrm{d} s=\infty\right) \in\{0,1\}
$$

Furthermore, condition (A.3) is also necessary for $\mathbb{P}\left(\int_{0}^{\infty} h\left(R_{s}\right) \mathrm{d} s=\infty\right)=1$ to hold.

Proof. See Theorem 2 in Engelbert and Schmidt (1987).

Proposition A.3. Let $R(\cdot)$ be a Bessel process with dimension $d=2$ and $R(0)=$ $r \geq 0$. For a measurable function $h:(0, \infty) \mapsto[0, \infty)$ we have

$$
\mathbb{P}\left(\int_{0}^{\infty} h\left(R_{s}\right) \mathrm{d} s=\infty\right)=1
$$

if and only if Lebesque measure of the set $\{h \neq 0\}$ is strictly positive. Moreover, the $0-1$ law holds

$$
\mathbb{P}\left(\int_{0}^{\infty} h\left(R_{s}\right) \mathrm{d} s=\infty\right) \in\{0,1\} .
$$

Proof. See Theorem 2 in Engelbert and Schmidt (1987). 